Chapter 11

\title{
Through vial impedance spectroscopy (TVIS): A novel approach to process understanding for freeze-drying cycle development
}

Geoff Smith and Evgeny Polygalov

\begin{tabular}{|l|l|}
\hline Author & Geoff Smith and Evgeny Polygalov \\
\hline Chapter title & $\begin{array}{l}\text { Chapter 11 Through vial impedance spectroscopy (TVIS): A novel approach } \\
\text { to process understanding for freeze-drying cycle development }\end{array}$ \\
\hline Book & $\begin{array}{l}\text { Lyophilization of Pharmaceuticals and Biologicals: New Technologies and } \\
\text { Approaches, Methods in Pharmacology and Toxicology }\end{array}$ \\
\hline Editors & Kevin R. Ward and Paul Matejtschuk \\
\hline Publication year & 2019 \\
\hline Publisher & Humana \\
\hline DOI & \begin{tabular}{l} 
https://doi.org/10.1007/978-1-4939-8928-7 11 \\
\hline First online
\end{tabular} \\
\hline
\end{tabular}

Cite as:

SMITH, G. and POLYGALOV, E., 2019. Chapter 11 Through vial impedance spectroscopy (TVIS): A novel approach to process understanding for freeze-drying cycle development. In: K. R. WARD and P. MATEJTSCHUK, eds, Lyophilization of Pharmaceuticals and Biologicals: New Technologies and Approaches. 1 edn. New York: Humana Press, pp. 241-290.

* Affiliation of the authors

Leicester School of Pharmacy, De Montfort University, Leicester, LE1 9BH, United Kingdom 


\title{
Chapter 11 \\ Through vial impedance spectroscopy (TVIS): A novel approach to process understanding for freeze-drying cycle development
}

\author{
Geoff Smith and Evgeny Polygalov
}

\begin{abstract}
Through vial impedance spectroscopy (TVIS) provides a new process analytical technology for monitoring a development scale lyophilization process, which exploits the changes in the bulk electrical properties that occur on freezing and subsequent drying of a drug solution. Unlike the majority of uses of impedance spectroscopy, for freeze-drying process development, the electrodes do not contact the product but are attached to the outside of the glass vial which is used to contain the product to provide a non-sample-invasive monitoring technology. Impedance spectra (in frequency range $10 \mathrm{~Hz}$ to $1 \mathrm{MHz}$ ) are generated throughout the drying cycle by a specially designed impedance spectrometer based on a $1 \mathrm{G} \Omega$ trans-impedance amplifier and then displayed in terms of complex capacitance. Typical capacitance spectra have one or two peaks in the imaginary capacitance (i.e. the dielectric loss) and the same number of steps in the real part capacitance (i.e. the dielectric permittivity). This chapter explores the underlying mechanisms that are responsible for these dielectric processes, i.e. the Maxwell-Wagner (space charge) polarization of the glass wall of the vial through the contents of the vial when in the liquid state, and the dielectric relaxation of ice when in the frozen state. In future work, it will be demonstrated how to measure product temperature and drying rates within single vials and multiple (clusters) of vials, from which other critical process parameters, such as heat transfer coefficient and dry layer resistance may be determined.
\end{abstract}

Key words: Impedance spectroscopy, Process-analytical-technology, PAT, Freeze-drying, Lyophilization, Maxwell-Wagner, polarization, Dielectric relaxation, Ice.

\section{G. Smith}

Leicester School of Pharmacy, De Montfort University, Leicester, United Kingdom

e-mail: gsmith02@dmu.ac.uk 


\section{Introduction}

A study of the electrical impedance of materials has been used for many years for the investigation of various processes associated with freeze drying. However, in the main, these studies have been restricted to the determination of critical temperatures. In one of the earliest reports, Greaves (1954) monitored the crystallization process through the measurement of changes in electrical conductivity at a single excitation frequency of an applied ac voltage. The basis for this assessment is that the formation of ice, within a solution, increases the viscosity while reducing the volume of the unfrozen (i.e. non-ice) fraction; and as a consequence the mobility of the ionic species within the unfrozen fraction decreases which in turn is reflected in a reduction of the measured electrical conductivity. Some years later, similar measurements were used to determine the eutectic temperature of a solution by measuring the electric resistance as a function of the temperature of a frozen material on reheating (P. P. DeLuca 1977, P. Deluca and Lachman 1965, Rey 1960a). A number of other authors have followed suit by using electrical thermal analysis techniques to measure the eutectic temperatures $\left(T_{\text {eu }}\right)$ of salts, sugars and the occasional drug substance (Table 1). The basis for the assessment is that the resistance of a solidified frozen mass suddenly decreases when the temperature is heated through the eutectic temperature, but this time as a consequence of the increased mobility of ions which results from a combination of reduced viscosity and increased volume of the non-crystalline (liquid) fraction. Single frequency measurements have also been widely employed for off-line characterization of the glass transition temperature $\left(T_{\mathrm{g}}\right)$ and/or collapse temperature $\left(T_{\mathrm{c}}\right)$ of a number of pharmaceutical relevant excipients such as sugars, polymers salt solutions and proteins (Table 2)

Various experimental approaches have been used for characterizing the temperature dependence of the passive electrical properties of frozen solutions, which combine an electrode system with a temperature sensor and a reheating regime from temperatures as low as $-100{ }^{\circ} \mathrm{C}$. The electrode designs range from a pair of rods to a pair of flat plates or a set of multiple inter-digitated "comb-like" electrodes mounted on a flat surface (Morris et al. 1994, Evans et al. 1995). In each system the temperature sensor (usually a platinum resistance thermometer) is mounted in close proximity to the electrodes. In the vast majority of these studies a single frequency voltage is applied (usually $1 \mathrm{kHz}$ ) and the ratio of the voltage amplitude $\left(V_{o}\right)$ to the current amplitude $\left(I_{o}\right)$ is used to determine the impedance magnitude $\left(|Z|=V_{o} / I_{o}\right)$ and the phase difference between the two vector quantities of voltage and current is used to define the phase angle (theta, $\vartheta$ ). In some studies (Her et al. 1994) the sensor is calibrated against a known solution resistivity (or conductivity) so that the measured electrical resistance of the frozen solution may also be expressed in terms their dimensionless property (i.e. resistivity). In other studies, attempting to measure the dielectric properties of the frozen solution (Morris et al. 1994) a range of discrete frequencies are employed to construct a dielectric spectrum of the real and imaginary permittivity against the log of the frequency of the applied field.

Only a few commercial systems are available for off-line and in-line applications. Lyotherm, from Biopharma Process Systems Ltd, is an integrated electrical impedance $(|Z| \sin \varphi)$ and differential thermal analyser (DTA) designed to measure the critical temperatures associated with: (1) the glass transition of the maximally concentrated solutes $\left(T_{\mathrm{g}}^{\prime}\right),(2)$ the melting of any eutectic mixture that has crystallized from solution on freezing $\left(T_{\text {eu }}\right)$, and (3) the melting of the ice fraction $\left(T_{\mathrm{m}}\right) \cdot|Z| \sin \varphi$ is the imaginary part of the complex impedance $\left(Z^{*}\right)$ otherwise known as the reactance. While it is restricted to the analysis of certain critical temperatures it nevertheless highlights the unique sensitivity of impedance measurements over thermal methods for characterizing phase behaviour in frozen aqueous solutions. For example, the glass transition $\left(T_{\mathrm{g}}^{\prime}\right)$ of $10 \% \mathrm{w} / \mathrm{v}$ sucrose formulation that was determined by this technology agrees with the published value $\left(T_{\mathrm{g}}^{\prime}=-32{ }^{\circ} \mathrm{C}\right)$ (Ward and Matejtschuk 2010). To the best of our knowledge, the LyoRx system (from Martin Christ $\mathrm{GmbH}$ ) is currently the only commercially available process analytical technology, based on electrical impedance that is used for in process measurements. This system monitors the temperature of the product and its electrical resistance 
(at a fixed frequency of $1 \mathrm{kHz}$ ) to deliver the freezing/eutectic points while enabling process optimization by automatically controlling the energy supply to the individual shelves during the main drying phase, which then reduces the risk of product collapse and thawing (Bandari et al. 2013). The main drawback of LyoRx is the invasive measurement principle involving immersion of the electrodes into the sample. More recently, a new method non-contact method has been proposed by Alkeev et al. (2015) which uses a capacitive sensor connected to a self-oscillating circuit for the purpose of monitoring the lyophilization of biological products.

Table 1 Eutectic temperature $\left(T_{\mathrm{eu}}\right)$ of various materials

\begin{tabular}{|c|c|c|c|}
\hline \multirow{2}{*}{ Test samples } & \multicolumn{2}{|c|}{$T_{\mathrm{eu}}\left({ }^{\circ} \mathrm{C}\right)$} & \multirow{2}{*}{ References } \\
\hline & ETA & DTA/DSC & \\
\hline $\mathrm{CaCl}_{2}$ & -54.0 & & (Ward and Matejtschuk 2010) \\
\hline $\mathrm{KBr}$ & -12.9 & & (P. Deluca and Lachman 1965) \\
\hline $\mathrm{KCl}$ & -11.1 & & (P. Deluca and Lachman 1965) \\
\hline Lactose & -5.4 & & (P. Deluca and Lachman 1965) \\
\hline Mannitol & $\begin{array}{l}-24.0 \\
-5.0 \\
-2.24 \\
\end{array}$ & $\begin{array}{c}-22.0 \\
-2.0\end{array}$ & $\begin{array}{l}\text { (Arshad et al. 2014) } \\
\text { (Evans et al. 1995) } \\
\text { (P. Deluca and Lachman 1965) }\end{array}$ \\
\hline Methylphenidate $\mathrm{HCl}$ & -11.7 & & (P. Deluca and Lachman 1965) \\
\hline Methylphenidate $\mathrm{HCl}$ with Lactose & -11.9 & & (P. Deluca and Lachman 1965) \\
\hline Methylphenidate $\mathrm{HCl}$ with mannitol & -11.7 & & (P. Deluca and Lachman 1965) \\
\hline Methylphenidate methanesulfonate & -10.1 & & (P. Deluca and Lachman 1965) \\
\hline Methylphenidate phosphate & -4.29 & & (P. Deluca and Lachman 1965) \\
\hline $\mathrm{NaCl}$ & $\begin{array}{l}-21.0 \\
-21.6 \\
-21.6 \\
-21.5\end{array}$ & $\begin{array}{l}-20.9 \\
-21.6\end{array}$ & $\begin{array}{l}\text { (Evans et al. 1995) } \\
\text { (P. Deluca and Lachman 1965) } \\
\text { (Rey 1960b) } \\
\text { (Rey 1960a) }\end{array}$ \\
\hline Phentolamine $\mathrm{HCl}$ & -1.3 & & (P. Deluca and Lachman 1965) \\
\hline Phentolamine methanesulfonate & -11.0 & & (P. Deluca and Lachman 1965) \\
\hline Phentolamine phosphate & -0.75 & & (P. Deluca and Lachman 1965) \\
\hline
\end{tabular}

ETA (electrical thermal analysis) is a generic term for a number of techniques that measure the electrical properties of a material as a function of temperature, including electrical resistivity measurements (ER), broadband dielectric spectroscopy (BDS), thermally stimulated current spectroscopy (TSC), and impedance spectroscopy (IS) DTA (differential thermal analysis) and DSC (differential scanning calorimetry) are thermal analysis techniques typically used for the determination of phase transitions. 
Table 2 Critical temperatures $T_{\mathrm{g}}^{\prime}$ and $T_{\mathrm{c}}$ of various materials

\begin{tabular}{|c|c|c|c|c|c|}
\hline \multirow{2}{*}{ Test samples } & \multicolumn{2}{|c|}{$T_{\mathrm{g}}^{\prime}\left({ }^{\circ} \mathrm{C}\right)$} & \multicolumn{2}{|c|}{$T_{\mathrm{c}}\left({ }^{\circ} \mathrm{C}\right)$} & \multirow[b]{2}{*}{ References } \\
\hline & ETA & $\begin{array}{l}\text { DTA/ } \\
\text { DSC }\end{array}$ & ETA & FDM & \\
\hline Azactam $^{\mathrm{TM}}$ & & & -17.7 & -17.0 & (Morris et al. 1994) \\
\hline Dextran $8.8 \mathrm{~K}$ & -14.2 & -16.5 & & & (Her et al. 1994) \\
\hline Dextran 39.1K & -13.2 & -15.4 & & & (Her et al. 1994) \\
\hline Dextran $70 \mathrm{~K}$ & -13.6 & -15.4 & & & (Her et al. 1994) \\
\hline Dextran $503 \mathrm{~K}$ & -13.0 & -15.0 & & & (Her et al. 1994) \\
\hline Dextrose (D-glucose) & -40.6 & -49.5 & & & (Her et al. 1994) \\
\hline Ficoll 70K & -22.6 & -23.9 & & & (Her et al. 1994) \\
\hline Ficoll 400K & -23.4 & -23.9 & & & (Her et al. 1994) \\
\hline Gelatin & -12.8 & -14.2 & & & (Her et al. 1994) \\
\hline Lactose & -29.5 & $\begin{array}{l}-30.3 \\
-33.4\end{array}$ & & & $\begin{array}{l}\text { (Ward and Matejtschuk 2010) } \\
\text { (Her et al. 1994) }\end{array}$ \\
\hline Maltodextrin & -16.0 & -17.0 & & & $\begin{array}{l}\text { (Smith, Arshad, Polygalov and } \\
\text { Ermolina 2014b) }\end{array}$ \\
\hline Mannitol & -33.7 & -33.9 & & & (Her et al. 1994) \\
\hline PVP $10 \mathrm{~K}$ & -28.3 & -32.3 & & & (Her et al. 1994) \\
\hline PVP $30 \mathrm{~K}$ & -22.0 & -22.8 & & -23.0 & (Mackenzie 1985) \\
\hline PVP 40K & -23.5 & -26.6 & & & (Her et al. 1994) \\
\hline Sorbitol & -41.6 & -52.1 & -47.2 & -45.0 & $\begin{array}{l}\text { (Morris et al. 1994) } \\
\text { (Her et al. 1994) }\end{array}$ \\
\hline Sucrose & $\begin{array}{l}-32.0 \\
-33.5 \\
\end{array}$ & -34.0 & -35.0 & -32.0 & $\begin{array}{l}\text { (Smith et al. 2013a) } \\
\text { (Ward and Matejtschuk 2010) } \\
\text { (Morris et al. 1994) } \\
\text { (Her et al. 1994) }\end{array}$ \\
\hline Trehalose & -31.2 & -34.4 & -33.4 & -32.0 & $\begin{array}{l}\text { (Morris et al. 1994) } \\
\text { (Her et al. 1994) }\end{array}$ \\
\hline
\end{tabular}

ETA (electrical thermal analysis) is a generic term for a number of techniques that measure the electrical properties of a material as a function of temperature, including electrical resistivity measurements (ER), broadband dielectric spectroscopy (BDS), thermally stimulated current spectroscopy (TSC), and impedance spectroscopy (IS)

DTA (differential thermal analysis) and DSC (differential scanning calorimetry) are thermal analysis techniques typically used for the determination of phase transitions.

FDM (Freeze Drying Microscope) is a technique for determining critical collapse temperature of freeze drying formulations 


\section{TVIS Method}

\subsection{TVIS Measurement System}

TVIS is a process analytical technology, for development scale application, which measures the electrical impedance spectrum of a conventional freeze-drying vial and its contents, using electrodes which are located outside the vial, hence the term "through-vial impedance spectroscopy". The technique is therefore non-invasive in the sense that the electrodes do not contact the sample.

The technology has been developed, in the first instance, with electrodes attached to the external surface of a conventional glass freeze-drying vial. To date, the majority of the published work has been undertaken on Type I glass vials (nominal capacity $10 \mathrm{~mL}$ ) with a single pair of electrodes, $5 \mathrm{~mm}$ in height, and with each surrounded by a guard electrode (Smith et al. 2017, Arshad et al. 2014, Smith et al. 2014, Smith, Arshad, Polygalov and Ermolina 2014a, Smith, Arshad, Polygalov and Ermolina 2014b, Smith et al. 2013a, Smith et al. 2013b).

In contrast, the majority of the data presented in this chapter has been generated using Type I glass vials (nominal capacity $10 \mathrm{~mL}$ ) with a single pair of electrodes, $10 \mathrm{~mm}$ in height, but without the guard electrodes that were used in previous studies (Fig. 1a). The absence of the guard electrode makes it more straightforward to attach electrodes to form the TVIS measurement vials, whereas the increased electrode height from $5 \mathrm{~mm}$ to $10 \mathrm{~mm}$ has the additional benefit of being able to use TVIS to measure drying rates for typical fill volumes between $2-3 \mathrm{~mL}$.
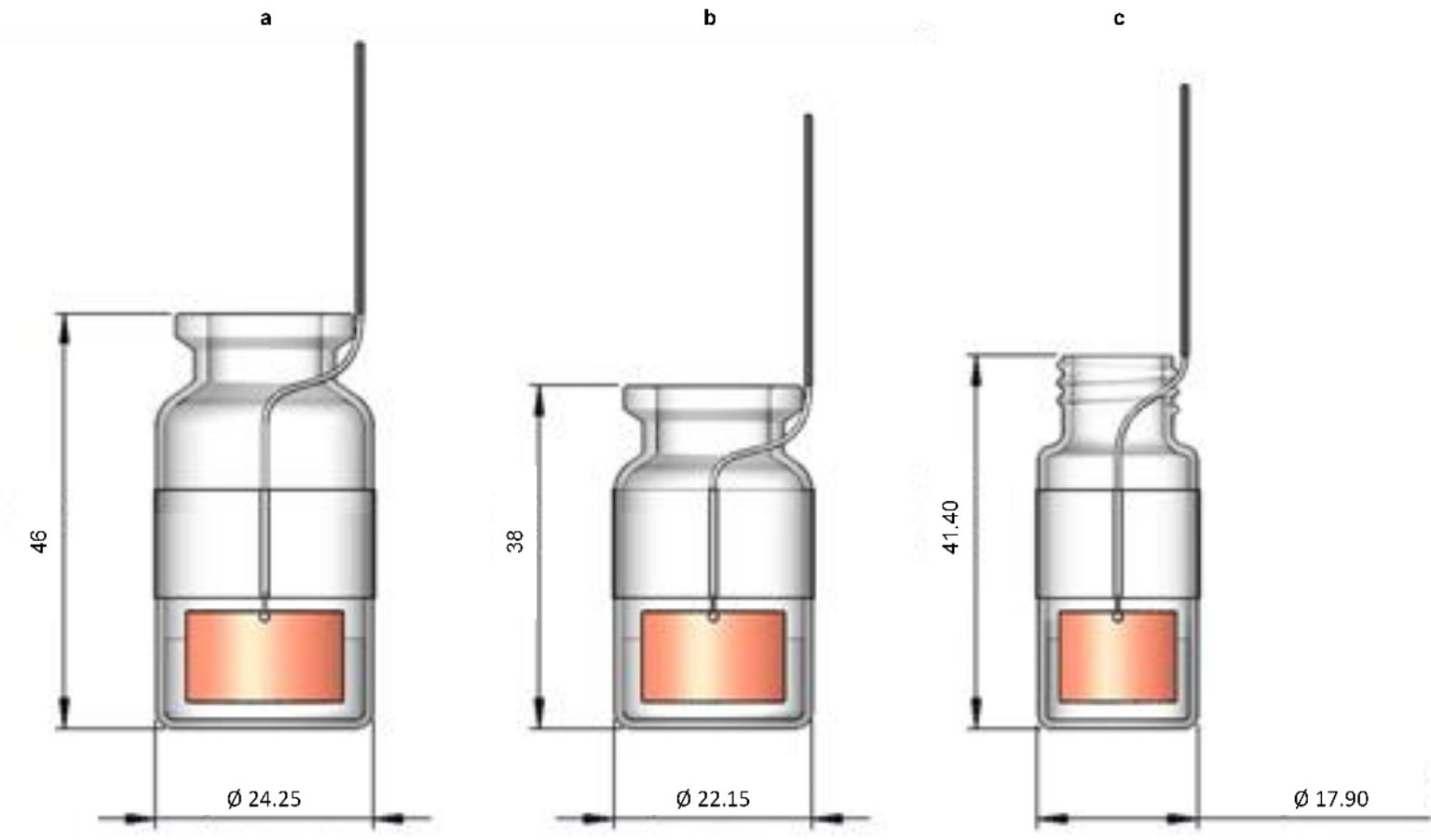

Fig. 1 Illustrations of the designs of various vials that have been modified with copper foil electrodes (10 $\mathrm{mm}$ in height and $3 \mathrm{~mm}$ from the base of each container). From left to right are non-ISO standard uncoated Type I borosilicate Fiolax ${ }^{\circledR}$ glass vials: (a) Adelphi VC010-20C (20 mm crimp-neck vial with $10 \mathrm{~mL}$ nominal capacity); (b) Adelphi VC005-20C (20 mm crimp-neck vial with $5 \mathrm{~mL}$ nominal capacity); (c) Adelphi VCD005 (screw-neck vial with $5 \mathrm{~mL}$ nominal capacity). Images have been provided courtesy of BlueFrog Design Ltd, Leicester, UK

Other vial types are possible (Fig. 1) with the only rule of thumb being that the combined width of the electrode pair does not occupy more than $50 \%$ of the vial circumference. The height of the 
electrode system, however, is variable, with the possibility for using tall electrodes which span the length of the vial. With the multichannel capability it is also possible to have multiple pairs of electrodes for predicting temperatures at the base of the ice $\left(T_{b}\right)$ and at the sublimation interface $\left(T_{i}\right)$.

When fitted to a commercial freeze-dryer, there is a requirement for a bespoke pass-through (Fig. 2) which carries the co-axial cabling from the impedance spectrometer, located outside the dryer, to the vial located inside the dryer.

a

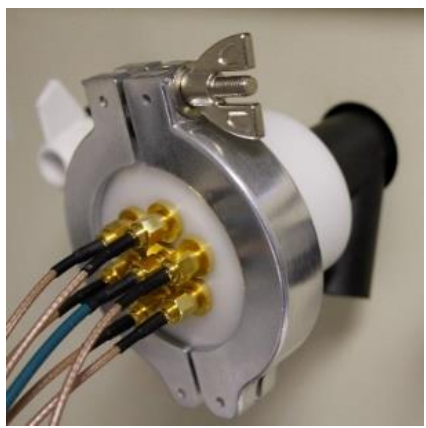

b

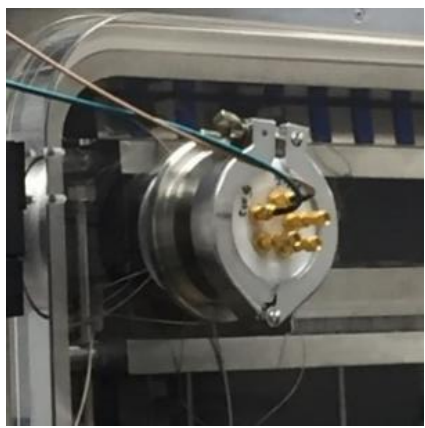

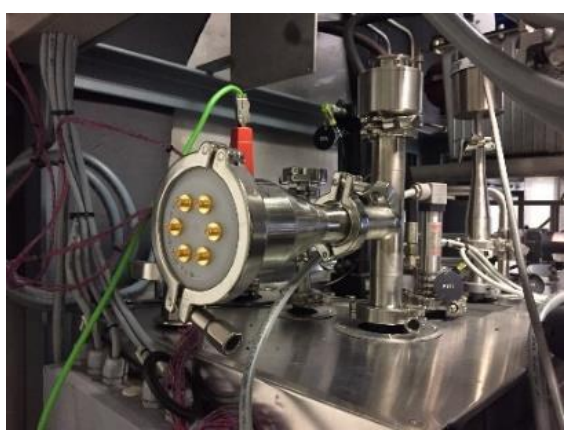

Fig. 2 Example pass-throughs for the TVIS instrument that have been deployed on a range of freezedryers at various institutions and companies:

(a) Virtis Advantage Plus XL freeze-dryer (De Montfort University) in which the pass-through is connected via the manifold hose on the outside of the dryer;

(b) Telstar LyoBeta freeze-dryer (Image courtesy of the National Institute of Biological Standards and Control) in which the pass-through is connected via the port on top left side of the door on the dryer;

(c) GEA LYOVACTM FCM2 LN2 pilot scale dryer (Image courtesy of GEA Lyophil GmbH) where the pass-through is connected to a port on the top of the drying chamber; Each pass-through has a minimum of six TE-connectivity-AMP SMA-jack to SMA jack bulk-head adapters (1054874-1): One adapter is used for the stimulating signal and the other five for the receiving signal. All pass-throughs were manufactured by GEA Pharma Systems (Eastleigh, UK)

The cabling and connections for the multichannel system, used to acquire the exemplar data for this book chapter, can be described as follows:

$50 \Omega 26$ AWG RG316 double-shielded co-axial cables take the stimulating voltage from the analogue output (AO1) of a National Instruments PCI6110 Multi-function I/O device (inside the tower of a desk-top PC) via a National Instruments BNC 2120 connector block, to one of the TE-connectivityAMP SMA-SMA jack adapters on the pass-through. On the inside of the dryer (i.e. on the other side of the pass-through) a $50 \Omega 30$ AWG RG178 co-axial cable takes the stimulating signal to a 5 way multiplexer inside the junction box (which is placed somewhere convenient within the dryer, Fig. 3). The splitter feeds the voltage, simultaneously, to five MCX coaxial stimulating ports on the top row of the junction box.

Any one of the two fine coaxial cables from the measurement vial is then connected to one of the MCX co-axial stimulating ports on the multiplexer and the other connected to the corresponding MCX coaxial sensing port on the bottom row of the junction box via the right-angle MCX $50 \Omega$ connector plugs (JOHNSON 133-3402-101) which terminate these cables. The coaxial cables attached to the measurement vial have an outer diameter of $0.533 \mathrm{~mm}$, with a 36 AWG tinned copper innerconductor, a 90\% spiral tinned copper braid screening and PFA polymer for both the external coating and insulation core. The cables are sufficiently flexible such that they don't apply any appreciable torsion to the vial that might otherwise cause the vial to tip or make it difficult to position on the shelf. 


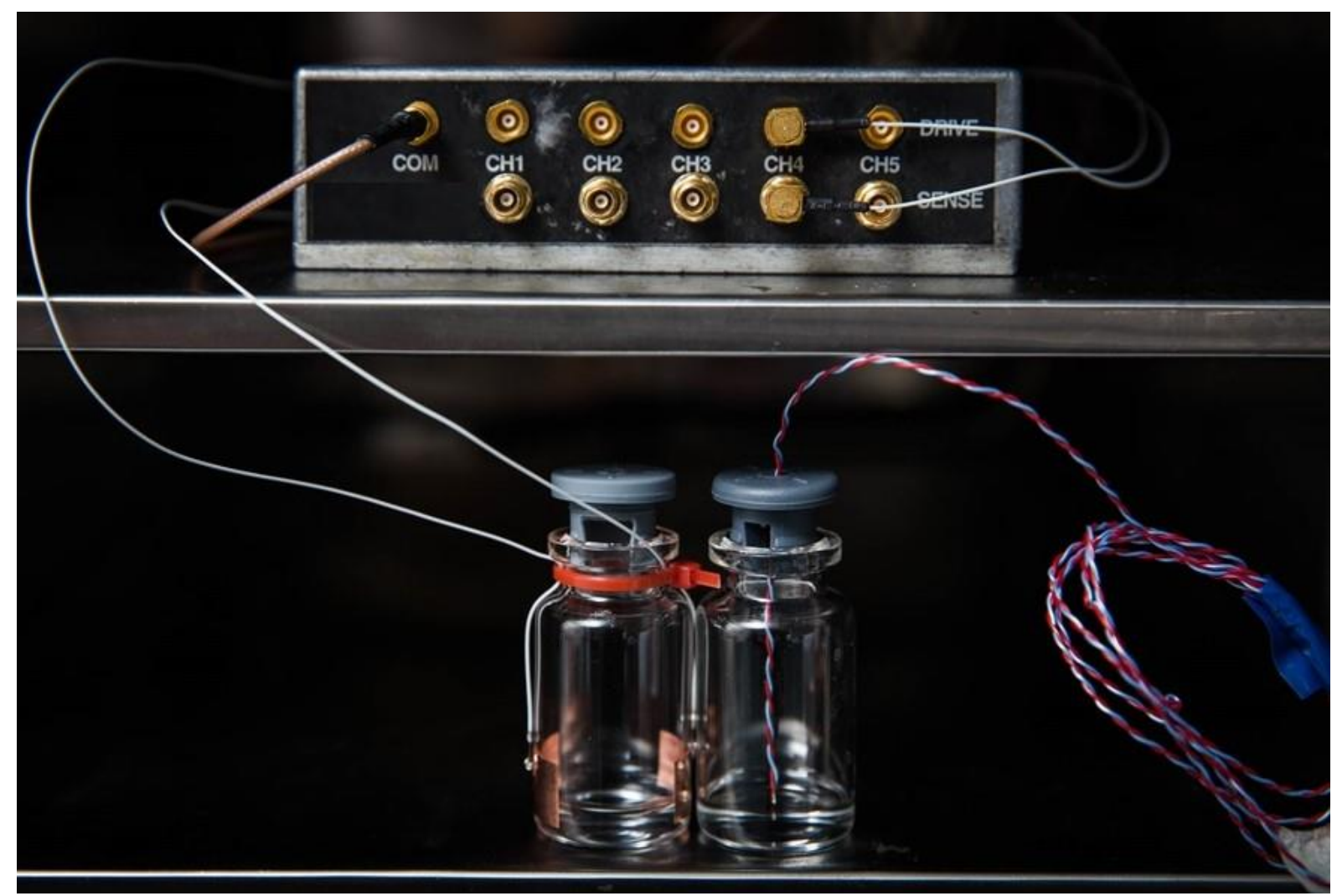

Fig. 3 Top shelf sits the 5 channel multiplexer. The COM channel receives the excitation signal from the NI DAQ PCI 6110 and distributes it to the stimulating ports on the top row of the multiplexer. The current passing through the vial flows through the receiving ports on the bottom row and then to the IVC outside the freeze-dryer where it is converted to a voltage and then passed to the NI DAQ PCI6110 in the computer and digitized. Bottom shelf (left) is the TVIS measurement vial. Bottom shelf (right) is a standard vial with a thermocouple inserted in the liquid such that the thermocouple bead is at a height which is equivalent to the mid-point of the volume of liquid in the region bounded by the electrodes in the TVIS vial (see Fig. 4 for an illustration).

The signals from the five sensing ports on the bottom row of the multiplexer are then passed to the five individual trans-impedance amplifiers inside the current to voltage (IVC) converter unit (located outside the dryer), again via pairs of $50 \Omega 30$ AWG RG178 and 26 AWG RG316 co-axial cables (with the RG178 cable inside the dryer and the RG316 on the outside of the dryer).

The stimulating signal is fed to the ADC0 input of the 4-channel NI DAQ PCI6110 Multifunction I/O device whereas the five outputs from the IVC channels are fed to the ADC1 input, via a programmatically controlled reed relay multiplexer.

All connections inside the dryer are made with Belden 30 AWG RG178 coaxial cables which are terminated by SMA plug connectors (HUBER \& SUHNER 11 SMA-50-1-4/111NE) on the pass through side and by MCX bulkhead Jack connectors (MULTICOMP 27-10M TGG) on junction box side. All outside connections are made with Belden coaxial cable 26 AWG RG316 terminated by SMA straight plug connectors (MULTICOMP MC23554). 


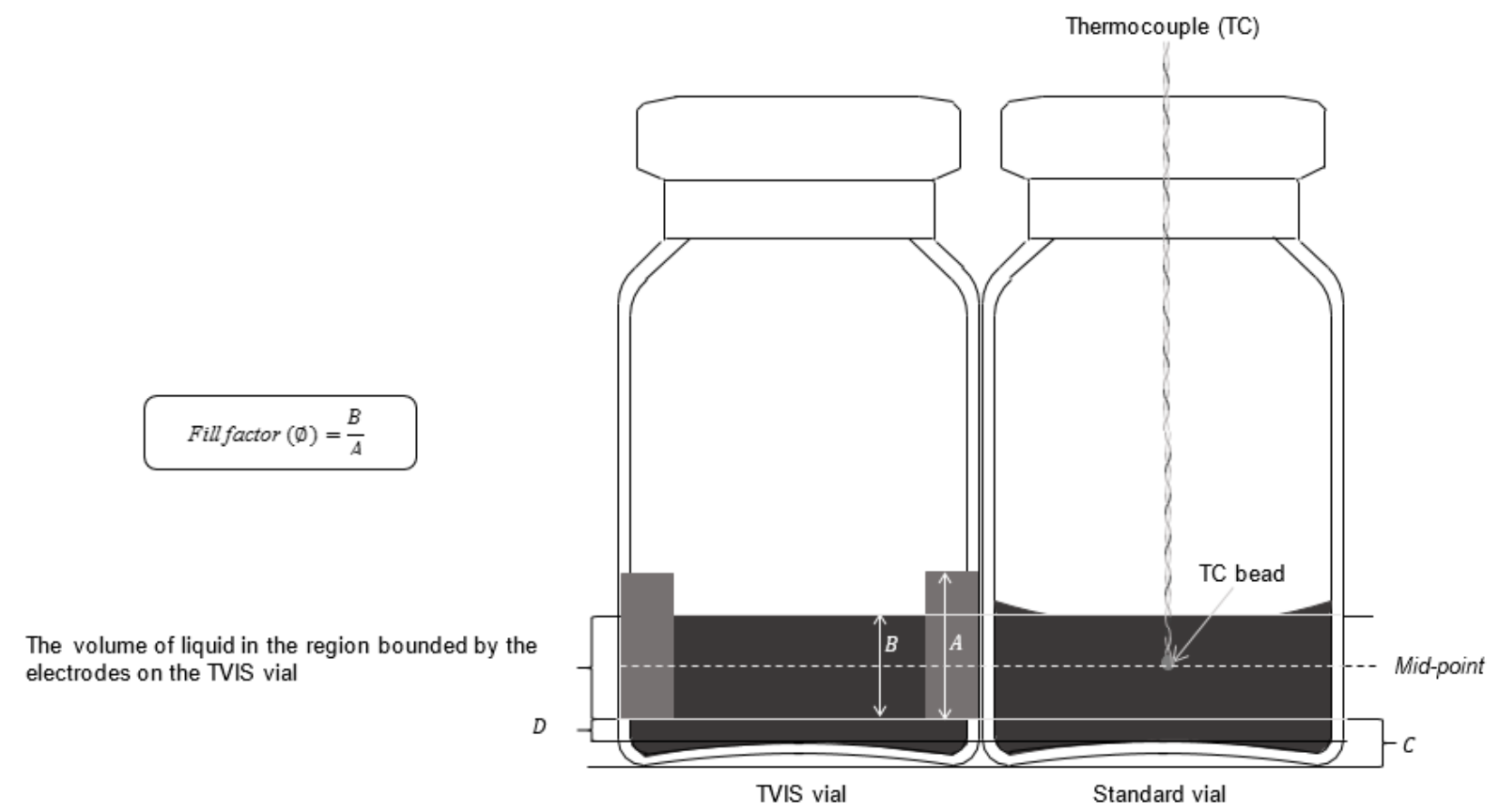

Fig. 4 Sketch of an Adelphi VC010-20C vial (20 mm crimp-neck vial with $10 \mathrm{~mL}$ nominal capacity and external diameter and internal diameters of $24.25 \mathrm{~mm}$ and $22.05 \mathrm{~mm}$ ); Right: Unmodified: Unmodified "nearest neighbor" vial. Left: TVIS-modified vial showing the electrode pair of height 10 $\mathrm{mm}$ and width $19 \mathrm{~mm}$, with a $3 \mathrm{~mm}$ separation between the bottom of the electrode and the external base of the vial (Dimension $C$ ). The Dimension $D$ is the approximate height of the liquid on circumference of the volume space below the lower edge of the electrode. Its magnitude is given by dimension $C$ minus the thickness of the base at the contact point with the external surface on which the vial is placed. The fill factor $\emptyset$ shown here is 0.7 (which, for a $10 \mathrm{~mm}$ high electrode, means that the height of the liquid within the region bounded by the electrodes is $7 \mathrm{~mm}$ ). The fill volume of water is approximately $3.4 \mathrm{~mL}$. The position of the thermocouple, inserted in a nearest neighbor vial to the TVIS vial, is at a height which is equivalent to the mid-point of the volume of the liquid that is bounded by the electrodes on either side of the glass wall of the TVIS vial.

The electrodes are formed from $19 \mathrm{~mm}$ wide copper adhesive tape $\left(11813 \mathrm{M}^{\mathrm{TM}}\right)$ consisting of a soft copper foil backing $(0.04 \mathrm{~mm})$ and an electrically conductive pressure-sensitive acrylic adhesive $(0.026 \mathrm{~mm})$ attached to the outer wall of the vial. This approach is only one of a number of possible design options. Others include foil electrodes with an adhesive "wrap-over" layer to avoid having glue between the electrode and the glass wall, and electrodes which are deposited directly onto the surface of the vial using electro-plating and electroless-plating technologies.

The location of the electrodes on the outside of the vial has a number of benefits:

1 It helps maintain a predefined and immovable geometry which then stabilizes the magnitude of the measured impedance for any particular fill volume and/or condition (phase state and temperature) of the sample.

2 The electrodes do not require a support mechanism which then reduces the thermal mass of the electrode assembly, whereas any attempt to create a low thermal mass electrode assembly which is inserted into the vial will necessarily be fragile and susceptible to deformation with consequent alterations in the measured impedance.

3 The high impedance of the glass wall, in series with the sample, means that any variation in the layout of the cabling has negligible impact on the measured impedance response. 
4 The electrode assembly does not provide additional (non-representative) nucleation sites for ice growth that would otherwise alter the ice crystal structure that develops during the freezing stage

5 The thin electrode on the measurement vial means that the vials can be arranged in the conventional hexagonal array, thereby maintaining the inherent heat transfer between vials, shelf and the gaseous environment.

The principle disadvantage is that, in its current manifestation, the method depends on cables to connect the electrodes on the vials to the measurement system which renders the system incompatible with an automatic vial-loading system.

The main challenge in making precise (low noise) measurements of the TVIS spectrum is the large dynamic impedance range encountered across individual spectra (from $10 \mathrm{~Hz}$ to $10 \mathrm{MHz}$ ) and across the entire freeze-drying cycle (Fig. 5) where the impedance magnitude can be as high as 10 to $100 \mathrm{G} \Omega\left(10^{10}\right.$ to $\left.10^{11} \Omega\right)$ at the low frequency end of the spectrum $(10 \mathrm{~Hz})$ for the vial at the end of drying (when the vial is effectively empty from an impedance point of view) to as low as $100 \mathrm{k} \Omega$ (105 $\Omega$ ) at the high frequency end of the spectrum when the vial is full with a liquid, coupled to the requirements for a short spectrum acquisition/scan time of the order of 10s so that spectra from multiple vials may be acquired sequentially in less than one minute. This functionality is not provided by the current state-of-the-art broad band dielectric spectrometers, which can take as long as one minute to acquire a single spectrum from $1 \mathrm{MHz}$ down to $10 \mathrm{~Hz}$, and so the implementation of the TVIS method has required the design and development of a bespoke instrument for this purpose.

\subsection{TVIS Measurement Instrumentation}

The instrument used to generate the data for this book chapter has been developed by Evgeny Polygalov at De Montfort University (Innovate UK project grant LyoDEA 100527). It is based on an NI PCI 6110 data acquisition (DAQ) card with a $4 \mathrm{MHz}$ sampling rate, a $1 \mathrm{G} \Omega$ trans impedance amplifier (i.e. a current to voltage convertor, IVC, with a trans-impedance gain of $180 \mathrm{~dB}$ ) and five sequentially measuring impedance channels which share a common excitation signal. The instrument features an automatic adjustment of the excitation signal, whereby the output level from the instrument, i.e. the amplitude of output signal from the IVC, is maintained at $2 \mathrm{~V}$, which is half of the maximum output level of $4 \mathrm{~V}$ so that the operational amplifier output doesn't experience any significant nonlinear distortion. This is achieved by adjustment of the stimulation voltage between 0.2 and $8 \mathrm{~V}$ and then fixing it at $8 \mathrm{~V}$ when the maintenance of this level is no longer achievable: In the sequence for the measurement of the first data point of each spectrum, the stimulating voltage is first set to $0.2 \mathrm{~V}$; the system then checks for an overflow/underflow of the ADC and changes the gain of the data acquisition card until the ADC is functioning in its optimal regime. Then the system checks the amplitude of IVC output and adjusts the amplitude of simulating signal in order to obtain the target $2 \mathrm{~V}$ output of the IVC. This procedure can have several iterations until the optimal regime is achieved and only then the first data point is taken. For the subsequent data points in the spectrum, the optimal excitation signal amplitude and ADC gain are predicted on the basis of previous point measurement parameters. The same applies to the measurement of data points for subsequent spectra.

Another feature of the instrument is the methodology for improving the signal to noise ratio: The instrument integrates (i.e. averages) a number of sine wave periods $(\mathrm{N})$; When the duration of that number of periods is longer than $0.05 \mathrm{~s}$ then $\mathrm{N}$ is fixed at a pre-set number ( 4 is recommended), otherwise $\mathrm{N}$ is calculated as $\mathrm{N}=0.05 \mathrm{~s} \times \mathrm{f} \mathrm{Hz}$. It follows that $\mathrm{N}=4$ for a frequency of $80 \mathrm{~Hz}$ and below; while at $100 \mathrm{~Hz}, \mathrm{~N}=5$ (i.e. $0.05 \mathrm{~s} \times 100 \mathrm{~Hz}$ ); at $1 \mathrm{kHz}, \mathrm{N}=50$ (i.e. $0.05 \mathrm{~s} \times 1000 \mathrm{~Hz}$ ); at $10 \mathrm{kHz} \mathrm{N}=500$ (i.e. $0.05 \mathrm{~s} \times 10,000 \mathrm{~Hz}$ ); and so on. One or more first periods (which can be pre-set in the "Delay" box in "Experiment setup" window) are excluded from averaging to avoid any dynamic distortions that result from the essentially reactive impedance of the object under test (OUT). The integration and delay 
times for each frequency point of the spectrum then define the total scan time to acquire each individual spectrum during the freeze-drying cycle. For a spectrum recorded from $1 \mathrm{MHz}$ to $10 \mathrm{~Hz}$, with 10 data points per decade, the instrument takes $\sim 4 \mathrm{~s}$ to scan from $1 \mathrm{MHz}$ to $100 \mathrm{~Hz}$ and then a further $\sim 3-4 \mathrm{~s}$ to scan from $100 \mathrm{~Hz}$ to $10 \mathrm{~Hz}$. So, the total time taken to scan across the whole range frequency range of $10 \mathrm{~Hz}$ to $1 \mathrm{MHz}$ is 7-8 seconds per channel. With the five channel instrument it is possible to measure five vials in sequence in less than $1 \mathrm{~min}$.

\subsection{TVIS Measurement Principles}

The electrical impedance of an object (in our case a freeze-drying vial and its contents) defines the total opposition to the flow of charge (current) when a sinusoidal alternating voltage is applied. Given that the glass vial and its contents conduct electricity somewhat like a capacitor (rather than a conductor) then it can be more intuitive to present the electrical impedance of this object in terms of an electrical capacitance. By varying the frequency of the applied voltage, typically between $10 \mathrm{~Hz}$ to 1 $\mathrm{MHz}$, the TVIS system generates an electrical impedance spectrum which when displayed in terms of the equivalent electrical capacitance spectrum is characterized by a peak in the imaginary capacitance (i.e. dielectric loss) spectrum and a step in the real part capacitance (i.e. dielectric permittivity) spectrum. This basic response is seen routinely when measuring pure water where the peak in the imaginary capacitance is located within the mid-range of the experimental frequency window of $10 \mathrm{~Hz}$ to $1 \mathrm{MHz}$. However, the exact position and amplitude of the loss peak depends on the physical state of sample (whether liquid or frozen), the temperature of whichever phase the sample is in, at any point in time during freezing, annealing and or primary drying, and the height of the frozen layer within the region bounded by the electrodes on the outside of the vial. In effect, any changes in the capacitance spectrum will mirror the condition of the solution throughout the lyophilization process and therefore TVIS may be used to monitor the physical state, composition and temperature of the material throughout the lyophilization process.

Figure 5 shows example spectra for the TVIS vial (Adelphi VC010-20C of nominal volume 10 $\mathrm{mL}$ ) containing $3.5 \mathrm{~g}$ water $\left(\right.$ at $20^{\circ} \mathrm{C}$ ), $3.5 \mathrm{~g}$ frozen water (ice at $-20{ }^{\circ} \mathrm{C}$ ) and empty. The impedance spectra on the left (displayed as Bode plots, i.e. log impedance magnitude and phase angle against log frequency) illustrate the extremely wide range of impedance values associated with the various transitions that occur on freeze-drying; from the initial liquid state to the frozen state, and then to the end state, once all the ice has been removed, to leave behind the empty cell. The capacitance spectra on the right illustrate how the amplitude $\left(C^{\prime \prime}{ }_{P E A K}\right)$ and frequency position $\left(F_{P E A K}\right)$ of the main dielectric loss peak, in the imaginary capacitance spectrum, and the increment in capacitance $\left(\Delta C^{\prime}\right)$ and high frequency capacitance $\left(C^{\prime}(\infty)\right)$ in the real part capacitance spectrum, change with those same transitions. For the TVIS vial containing liquid water there is a single peak located at the high frequency end of the dielectric loss spectrum $(\sim 18 \mathrm{kHz})$, whereas for the TVIS vial containing frozen water (ice at $\left.-20^{\circ} \mathrm{C}\right)$ the peak has now shifted to lower frequencies of the dielectric loss spectrum $(\sim 3 \mathrm{kHz})$. There is also a dramatic decrease in the high frequency capacitance $\left(C^{\prime}(\infty)\right)$ in the real part spectrum. 

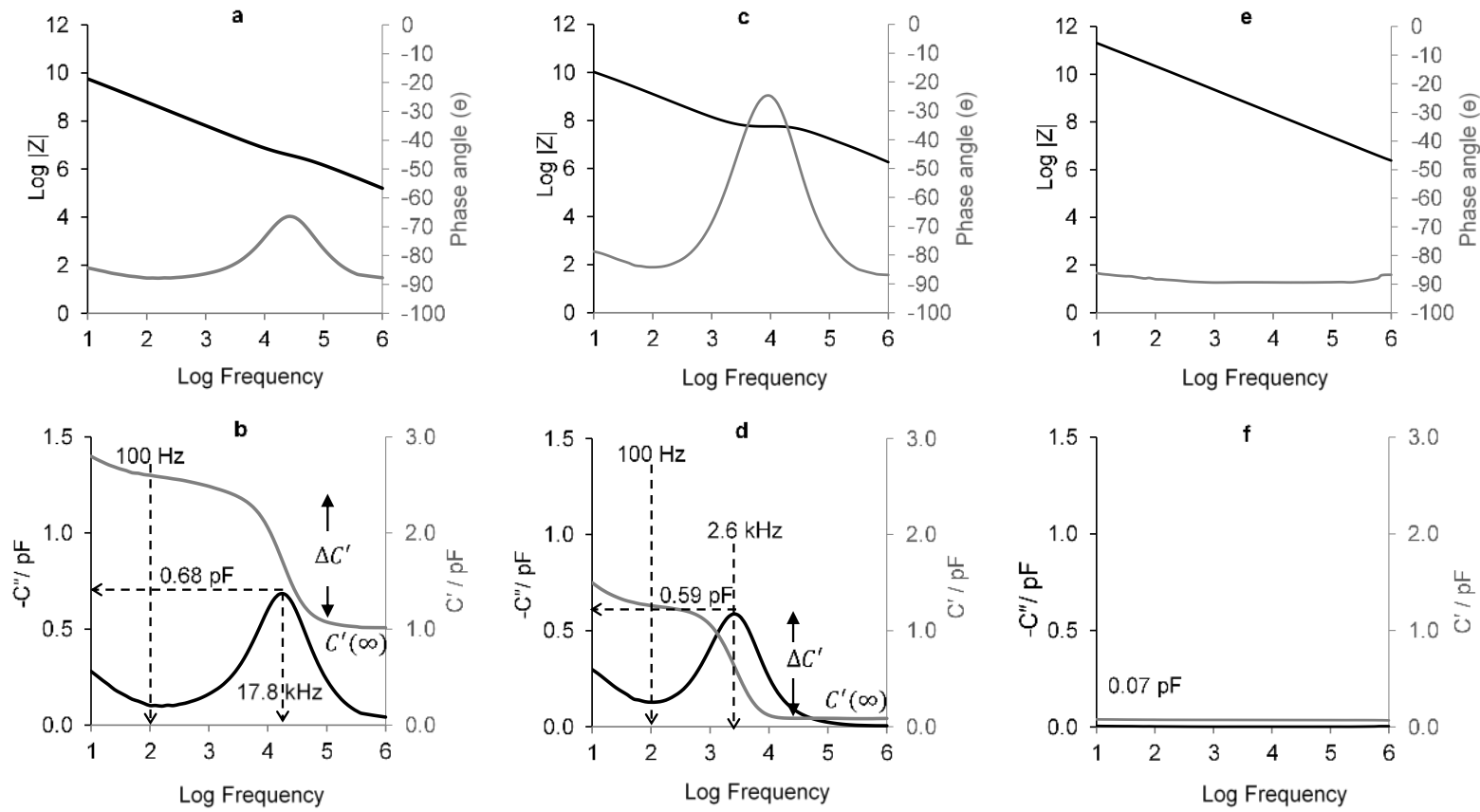

Fig. 5 a impedance spectrum and $\mathbf{b}$ equivalent complex capacitance spectrum of $3.5 \mathrm{~mL}$ double distilled water in an Adelphi VC010-20C Type I glass tubing vial with a pair of $10 \times 19 \mathrm{~mm}$ electrodes ( $\mathrm{h} \mathrm{x}$ w) attached to the outside of the vial at a distance of $3 \mathrm{~mm}$ from the external base $(\varnothing=0.7)$, $\mathbf{c}$ impedance spectrum and $\mathbf{d}$ equivalent complex capacitance spectrum of the same vial containing water but frozen to $-20{ }^{\circ} \mathrm{C} \mathbf{e}$ impedance spectrum and $\mathbf{f}$ equivalent complex capacitance spectrum of the same vial but without the sample. $|Z|$ is the magnitude of the impedance and $\vartheta$ is the phase angle between the real and imaginary parts of the complex impedance, $Z^{*} . C^{\prime}$ is the dielectric storage and $C^{\prime \prime}$ is the dielectric loss components of the complex capacitance, respectively. The vertical dotted line at $100 \mathrm{~Hz}$ illustrates the frequency below which both the real and imaginary capacitances increase as a consequence of charge percolation through the porous nature of the glass

\subsubsection{Liquids and the Maxwell-Wagner Polarization of the Glass}

In the case of simple, unfrozen liquids of relevance to pharmaceutical freeze-drying, such as water, ethanol, and tert-butanol, the peak in the imaginary capacitance (i.e. the dielectric loss) and the step in the real part capacitance (i.e. the dielectric storage) are due to a Maxwell-Wagner (MW) polarization process, otherwise known as space charge polarization or interfacial polarization. The MW process originates from the fact that the object under test is a composite of a relatively conductive material (i.e. the cylinder of liquid within the vial) in intimate contact with the poorly conductive material (i.e. the glass wall of the container). This particular type of MW process is manifest at the macroscopic scale of the glass-sample interface (i.e. ions migrate through the solution and accumulate at the interface between the solution and the glass). It should be stressed that the observation of the frequency dependent response of the composite object doesn't mean that the dielectric properties of the liquid has any frequency dependence of its own. The dielectric permittivity of water, for example, is constant within the frequency range of the TVIS instrument $(10 \mathrm{~Hz}$ to $10 \mathrm{MHz})$ and its conductivity can be described by a simple dc conductivity.

When a MW process occurs at the intermediate (meso-) scale (i.e. within the sample) because of inner dielectric and/or conductive boundaries which trap charge carriers within the material, then the object may display dielectric and conductive properties which are frequency dependent. This is a feature of many composite and microstructured materials, including: 
1 Multilayered polycrystalline composites (Shen et al. 2001) owing to the juxtaposition of thin films of alternating dielectric and conductive properties.

2 Ceramics (Liu et al. 2005) (e.g. as a result of grains and grain boundaries within the bulk material.

3 Biological cells and tissues (Stubbe and Gimsa 2015, Foster K.R. 1989) as a consequence of the relatively non-conductive (dielectric) property of the cytoplasmic membrane and the high conductivity of the intra- and extracellular fluid.

The physical origin and characteristics of the MW process (observed for the TVIS vial containing liquid water) can be considered as follows: When a voltage $(V)$ is applied to the composite object of the freeze-drying vial and its contents, via the electrodes attached to the outside of the vial, then current will start to flow and the electrical capacitance of the glass wall begins to accumulate charge at a rate which is dependent largely on the electrical resistance of the solution inside the vial. ${ }^{1}$

The charges accumulating at the glass boundary layer with the solution are neutralized to some degree by the accumulation of ionic charges that have diffused through the solution contained within the vial to the solution boundary layer with the glass. The net result is that a proportion of the applied voltage is dropped across the glass wall and the remaining voltage is dropped across the material within the vial.

According to classical electric circuit theory, the time constant $(\tau)$ for the rate of charging of the glass wall capacitance, through the solution resistance, is defined as the time it takes to reach $63.2 \%$ of its maximum charge holding capacity. This time constant may be approximated by the product of the sample resistance $\left(R_{\mathrm{S}}\right)$ and the glass-sample capacitance $\left(C_{\mathrm{g}}\right)$,

$$
\tau \approx R_{\mathrm{s}} C_{\mathrm{g}}
$$

One can rationalize the dependency of the time constant on both parameters, $R_{\mathrm{S}}$ and $C_{\mathrm{g}}$, by considering that it is the sample resistance that regulates the rate of flow of charges to the capacitance of the glass wall (hence restricting the rate of charging) whereas it is the capacitance of the glass wallsample interface which defines the amount of charge that the glass can accommodate (hence defining the total magnitude of charges that must first pass through the solution resistance for the capacitor to become fully charged). And so it follows that if, either the resistance or the capacitance increases, then it will take longer for the charging process to complete and that this would be reflected the value of the time constant $(\tau)$.

One should also recognize that the solution is not simply behaving as an electrical resistor it also has an electrical capacitance owing to the fact that the space between the two glass walls has a dielectric (charge storage) property which is due to the polarization of permanent dipoles (e.g. the molecular dipole of water and other small molecules). The actual time constant for the process is impacted by the additional time required for the solution capacitance to charge, such that

$$
\tau=R_{\mathrm{s}}\left(C_{\mathrm{g}}+C_{\mathrm{s}}\right)
$$

\footnotetext{
${ }^{1}$ Note that the impact on the flow of charge, from the cabling from the measurement instrument and the vial, is negligible given that the cables are made from conductive metal of significant lower resistance than that of the sample within the vial (i.e. the liquid/frozen solution or pure water/ice). This is one of the intrinsic benefits of the TVIS approach over instruments which place the electrodes in contact with the sample.
} 
where $C_{\mathrm{s}}$ is the approximate value for the electrical capacitance of the liquid contained primarily within the volume of the vial that is bounded by the electrodes but with a lesser contribution coming from the volume of liquid that is within the fringing fields extending beyond the boundaries of the electrodes.

\subsubsection{Impedance Spectroscopy}

The above treatment of the composite object of the glass wall and the liquid contents of a freezedrying vial considers the response of the object to a static (i.e. time invariant) voltage. However, when studying the charging of the glass wall through the solution resistance, using impedance spectroscopy, an oscillating sinusoidal voltage, $V=V_{\mathrm{o}} \sin (\omega t)$, is applied and the resultant current $I=I_{\mathrm{o}} \sin (\omega t+\varphi)$ is measured, where $\omega$ is the frequency of the applied field in radians and $\varphi$ is the phase difference between the voltage and current (Jacob 2004). The phase difference for a pure resistor is zero and that for a pure capacitor is $-90^{\circ}$ or $-\pi / 2$ radians (The negative sign means that the voltage lags behind the current by $\left.90^{\circ}\right)$. The ratio of the voltage amplitude $\left(V_{o}\right)$ to the current amplitude $\left(I_{o}\right)$ is known as the impedance magnitude, $|Z|=V_{o} / I_{o}$ whereas the inverse ratio $\left(I_{o} / V_{o}\right)$ is known as the admittance, $|Y|=1 /|Z|$.

\subsubsection{Basic Model for the TVIS Spectrum of the Liquid State}

The interpretation of impedance spectra is facilitated by using an equivalent circuit comprising individual elements that have characteristics which collectively reflect the physicality of the object while modelling the impedance of the object as a function of the applied frequency. For example, an object that is a good conductor is represented by a resistor whereas an object that doesn't conduct well (i.e. an insulator) is represented by a capacitor. The basic characteristics of the TVIS vial, comprising a non-conductive glass wall and a relatively conductive sample, have already been discussed in the previous section, and so now it is only a question of arranging these elements in an appropriate circuit layout which reflects the pathways for the flow of charge (i.e. current) (Fig. 6).

In the model for the liquid filled vial the electrical impedance of the solution is represented by a resistor $\left(R_{\mathrm{S}}\right)$ and capacitor $\left(C_{\mathrm{s}}\right)$ in parallel with each other, which reflects the fact that the solution has a measureable conductivity associated with delocalized charges, i.e. ions (which can diffuse through the solution) as well as a dielectric constant, which is associated with the polarization of charges that are more localized (i.e. molecular dipoles). The arrangement of $R_{\mathrm{S}}$ and $C_{\mathrm{S}}$ in parallel reflects the fact that current can flow independently as a result of the admittance of each component. ${ }^{2}$ The final element in the model is a capacitor in series with the sample impedance, which accounts for the dielectric behavior of the glass wall of the vial. Each of these impedance elements $\left(C_{\mathrm{s}}, R_{\mathrm{s}}, C_{\mathrm{g}}\right)$ will be considered in turn:

\footnotetext{
${ }^{2}$ Whilst it does not matter which element is placed above the other in a parallel circuit, we have adopted a rule that the element which dominates the impedance of the $\mathrm{R}=\mathrm{C}$ circuit at high frequency is placed above the element which dominates the impedance of the $\mathrm{R}=\mathrm{C}$ circuit at low frequency. Note that the use of the symbol "=" signifies a parallel arrangement of two elements, whereas the same elements in series will be indicated by the use of the symbol "-".
} 

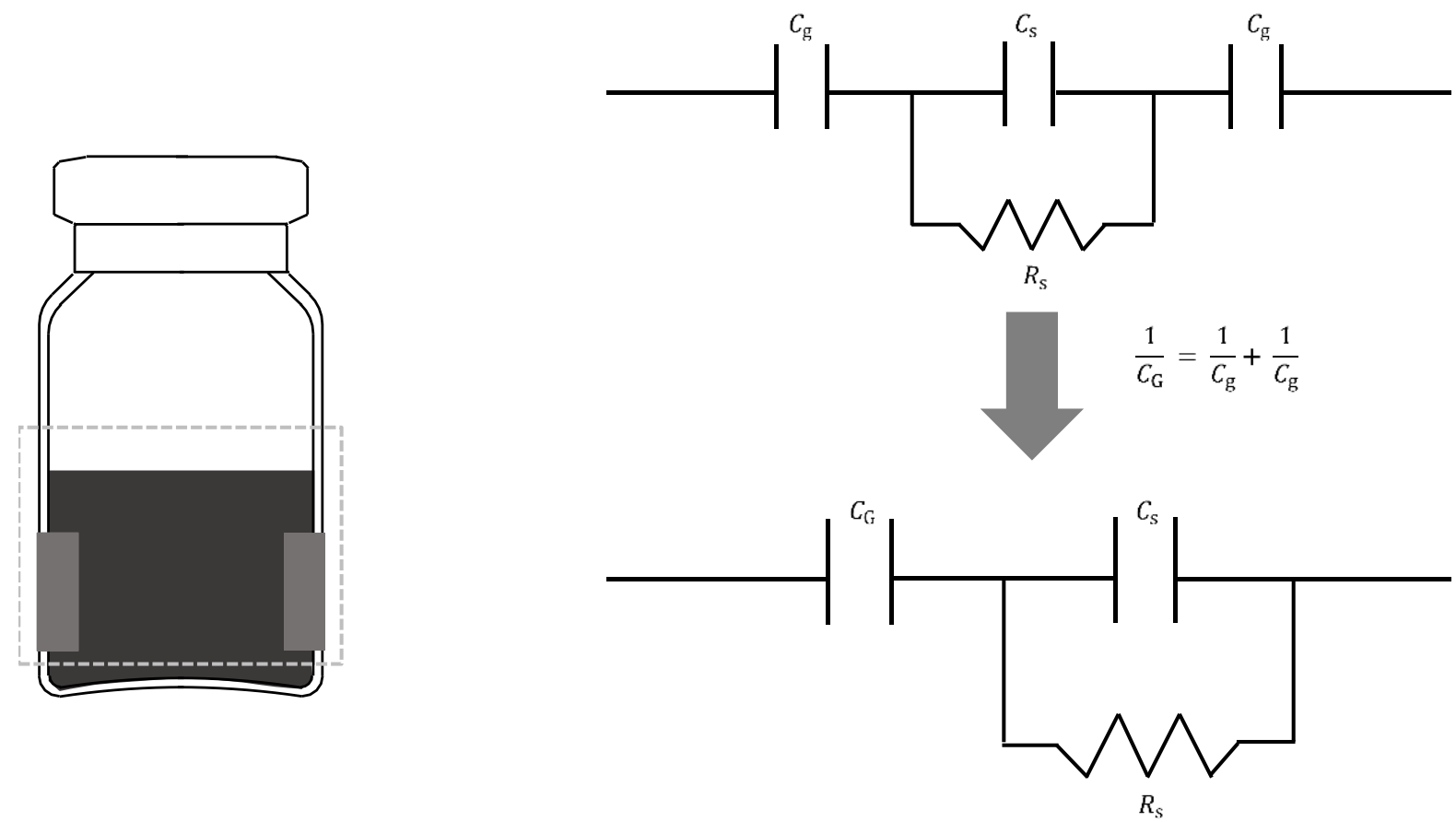

Fig. 6 Basic equivalent circuit model that approximately fits the experimental data for simple systems such as a water filled vial with copper foil electrodes attached to the outside of the glass. $C_{\mathrm{g}}$ is an approximation for the capacitance of the glass wall-sample interface and $C_{\mathrm{S}}$ and $R_{\mathrm{S}}$ are the capacitance and resistance of the sample respectively. The interface capacitances of the two segments of glass juxtaposed with the electrodes can be combined into a single capacitance using the inverse sum rule for capacitances in series, where the reciprocal of the total capacitance equals the sum of the reciprocal values of the two individual capacitances $1 / C_{\mathrm{G}}=1 / C_{\mathrm{g}}+1 / C_{\mathrm{g}} ; C_{\mathrm{G}}=C_{\mathrm{g}}^{2} /\left(C_{\mathrm{g}}+C_{\mathrm{g}}\right)$. Note the use of capital subscript $G$ for the combined capacitance from two glass segments in contact with the external electrodes.

The sample capacitance $\left(C_{\mathrm{s}}\right)$ is largely defined by the static permittivity $\left(\varepsilon_{S}\right)$ of the liquid (e.g. water) and the height of the liquid according to Eq. (3:

$$
C_{\mathrm{s}}=\varepsilon_{o} \varepsilon_{s} k_{s}
$$

where $\varepsilon_{o}$ is the permittivity of free space $\left(8.854 \times 10^{-12} \mathrm{~F} \cdot \mathrm{m}^{-1}\right)$ and $k_{s}$ is the geometric cell constant. The value of $k_{s}$ is dominated by the volume of liquid in the region of the vial bounded by the electrodes but with a smaller contribution from the liquid in the fringing fields of the electrodes. In the case of a parallel plate capacitor (with two planar electrodes of equal size and in close proximity to one another relative to their surface area) the cell constant $k_{S}$ would be defined by $A / d$, where $A$ is the area of an electrode and $d$ is the separation between the electrode pair. In the case of two curved electrodes, on opposite sides of a cylindrical object, then it is more difficult to define the cell constant in these simple geometric terms, owing to the fact that the fringing field effect is more significant the further apart the electrodes are. The characteristics of these fringing fields are illustrated later in Fig. 9.

The sample resistance $\left(R_{\mathrm{s}}\right)$ is largely defined by the concentration and valency (charge carrying capacity) of ions in the solution, according to Eq. 4:

$$
R_{\mathrm{s}}=\rho_{s} / k_{s}
$$



capacitance.

where $\rho_{s}$ is the resistivity of the solution and $k_{s}$ is the same geometric cell constant as for the

The high frequency contribution to the glass wall capacitance $\left(C_{\mathrm{g}}\right)$ is associated with the instantaneous atomic and electronic polarizations that create a macroscopic dipole moment which largely defines the instantaneous (i.e. frequency independent) relative permittivity of the glass $\left(\varepsilon_{g}\right)$ and hence its electrical capacitance. An analogous equation to that for the static sample capacitance of the sample may be applied to the instantaneous capacitance of the glass wall $\left(C_{\mathrm{g}}\right)$.

$$
C_{\mathrm{g}}=\varepsilon_{o} \varepsilon_{g} k_{g}
$$

The relative permittivity of the Fiolax ${ }^{\circledR}$ glass used for the Adelphi VC010-20C vial (which comprises $75 \% \mathrm{SiO}_{2}, 10.5 \% \mathrm{~B}_{2} \mathrm{O}_{3}, 5 \% \mathrm{Al}_{2} \mathrm{O}_{3}, 7 \% \mathrm{Na}_{2} \mathrm{O}$ and $1.5 \% \mathrm{CaO}$ ) is reported by the manufacturer (Schott) to be 5.7 at $1 \mathrm{MHz}\left(25^{\circ} \mathrm{C}\right)$. Whether this value is representative of the instantaneous relative permittivity of this glass remains to be seen. $k_{g}$ is the geometric cell constant of the segment of glass in direct contact with the electrodes. By ignoring edge effects (i.e. the fringing fields) then the cell constant may be estimated from $k_{g}=h \cdot w / d_{g}$, where $d$ is the thickness of the glass $\left(d_{g}=1.1 \mathrm{~mm}\right.$ for the Adelphi VC010-20C vial) and the area of the electrode is $(h \cdot w)$ is $190 \mathrm{~mm}^{2}(10 \mathrm{~mm} \times 19 \mathrm{~mm})$. However, given that the glass segment on which the electrode is attached comprises a curved surface rather than a planar one (Fig. 7), then the effective width of the glass segment that contributes to the cell constant is more likely to be closer to the average of the length of the external arc $(19 \mathrm{~mm})$ and the internal arc $(17.28$ $\mathrm{mm}$ ) of the glass segment, i.e. $w=18.14 \mathrm{~mm}$.

In a further refinement of the calculation of the theoretical capacitance of the glass-wall, one should also allow for the influence on the effective capacitance of the adhesive layer $\left(C_{\mathrm{a}}\right)$ in the composite assembly by applying the inverse sum rule of two capacitors in series (Carlson and Illman 2002).

$$
\begin{gathered}
\frac{1}{C_{\mathrm{a}-\mathrm{g}}}=\frac{1}{C_{\mathrm{a}}}+\frac{1}{C_{\mathrm{g}}} \\
C_{\mathrm{a}-\mathrm{g}}=\frac{C_{\mathrm{a}} \cdot C_{\mathrm{g}}}{C_{\mathrm{a}}+C_{\mathrm{g}}}
\end{gathered}
$$

In each case, the capacitance of the respective elements is given by an appropriate form of the parallel plate capacitor equation $\left(C=\varepsilon_{o} \varepsilon_{r} k\right)$ such that $C_{\mathrm{a}}=197.7 \mathrm{pF}$ and $C_{\mathrm{g}}=8.32 \mathrm{pF}$. The estimated capacitance $\left(C_{\mathrm{a}-\mathrm{g}}\right)$ of the composite of the glass wall and adhesive segment is therefore $7.98 \mathrm{pF}$. Therefore, a predicted value of $C_{\mathrm{a}-\mathrm{G}}$ would be $C_{\mathrm{a}-\mathrm{g}} / 2=3.99 \mathrm{pF}$. 


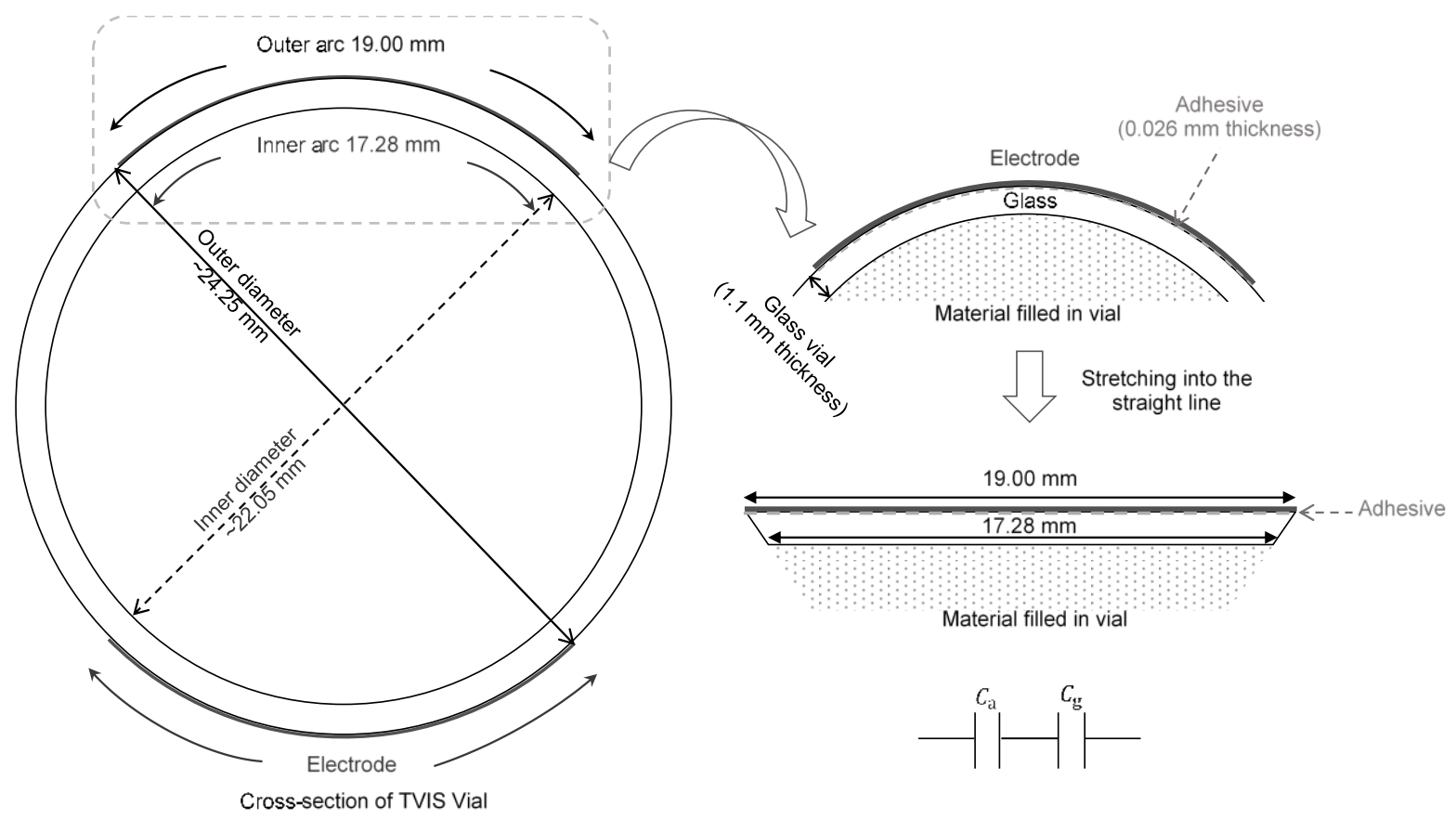

Fig. 7 Schematic showing the methodology for the estimation of the glass wall capacitance $\left(C_{\mathrm{g}}\right)$ from the geometric shape of the segment of the glass bounded by the external electrode and the literature value for the dielectric constant of the Fiolax ${ }^{\circledR}$ glass $\left(\varepsilon_{g}=5.7\right)$ and the adhesive $\left(\varepsilon_{a}=3.2\right)$ The curvature of glass vial and the dielectric constant of adhesive are taken into account for the calculation of the capacitance of the glass-composite segment $\left(C_{\mathrm{g}}=8.32 \mathrm{pF}, C_{\mathrm{a}}=197.7 \mathrm{pF}, C_{\mathrm{a}-\mathrm{g}}=7.98 \mathrm{pF}\right)$ with an electrode of dimension $10 \times 19 \mathrm{~mm}$ )

Figure 8 shows a typical capacitance spectrum for a TVIS-modified Adelphi VC010-20C glass tubing vial (nominal fill volume $10 \mathrm{~mL}$ ) containing $7 \mathrm{~g}$ double distilled water and with a pair of external electrodes of height $10 \mathrm{~mm}$ and width $19 \mathrm{~mm}$ (separated from the base of the vial by $3 \mathrm{~mm}$ ). The points on the graph are the data points $\left(C^{\prime}\right.$ and $\left.C^{\prime \prime}\right)$ recorded by the TVIS instrument and the line is the result of fitting the equivalent circuit in Fig. 6 using proprietary software (e.g. RelaxIS or Zview ${ }^{\circledR}$ ), to give $C^{\prime}{ }_{\text {fit }}$ and $C^{\prime \prime}{ }_{\text {fit }}$.

In a number of applications for TVIS, for example the determination of the product temperature, the extent of data analysis maybe restricted to the scrutiny of the amplitude of the dielectric loss peak $\left(C^{\prime \prime}{ }_{P E A K}\right)$ and the frequency on which the loss peak is centered $\left(F_{P E A K}\right)$. This provides a more pragmatic approach to the otherwise more complex procedures involved in fitting an equivalent circuit model to the data. However, in order to extract some physical meaning from these empirical parameters, it is necessary to consider the relationships between the empirical parameters $\left(C^{\prime \prime}{ }_{P E A K}\right.$ and $\left.F_{P E A K}\right)$ and the underlying changes in the equivalent circuit elements $\left(C_{\mathrm{S}}, R_{\mathrm{S}}, C_{\mathrm{G}}\right)$ that define the characteristics of the impedance spectrum These relationships will be explored here for the case of a simple solvent, i.e. water. Further basic explanations of impedance spectroscopy, as it applies to a liquid filled TVIS vial, is given in the appendix.

Figure 8a shows the imaginary-part capacitance (i.e. dielectric loss) spectrum of the TVIS vial containing liquid water. As the frequency tends towards zero $(\omega \rightarrow 0)$ the value for $C^{\prime \prime}$ fit from the equivalent circuit model also tends to zero. As the frequency increases, $C^{\prime \prime}{ }_{f i t}$ increases to a maximum $\left(C_{P E A K}^{\prime \prime}\right)$ at a frequency $F_{P E A K}$ then decreases to zero as the frequency increases above $F_{P E A K}$ (i.e. $\omega \rightarrow \infty)$. 


$$
\begin{gathered}
C_{P E A K}^{\prime \prime}=\frac{C_{\mathrm{G}}^{2}}{2\left(C_{\mathrm{s}}+C_{\mathrm{G}}\right)} \\
F_{P E A K}=\frac{1}{2 \pi R\left(C_{\mathrm{s}}+C_{\mathrm{G}}\right)}
\end{gathered}
$$

The corresponding real part is shown in Fig. 8b. In the limit of low frequency $(\omega \rightarrow 0)$ the real part capacitance of the equivalent circuit, $C_{f i t}^{\prime}$, tends towards a value equal to the capacitance of the glass-sample interface, i.e. $C_{\mathrm{G}}$. As the frequency increases $(\omega \rightarrow \infty)$ then $C^{\prime}{ }_{f i t}$ decreases to a plateau of

$$
C_{f i t}^{\prime}(\infty)=\frac{C_{\mathrm{s}} C_{\mathrm{G}}}{\left(C_{\mathrm{s}}+C_{\mathrm{G}}\right)}
$$

Note that the increment in the real part capacitance, $\Delta C^{\prime}$ (Eq. (11) is twice that of the amplitude of the dielectric loss peak (Eq. 8).

$$
C_{f i t}^{\prime}(\infty)=\frac{C_{\mathrm{s}} C_{\mathrm{G}}}{\left(C_{\mathrm{s}}+C_{\mathrm{G}}\right)}
$$

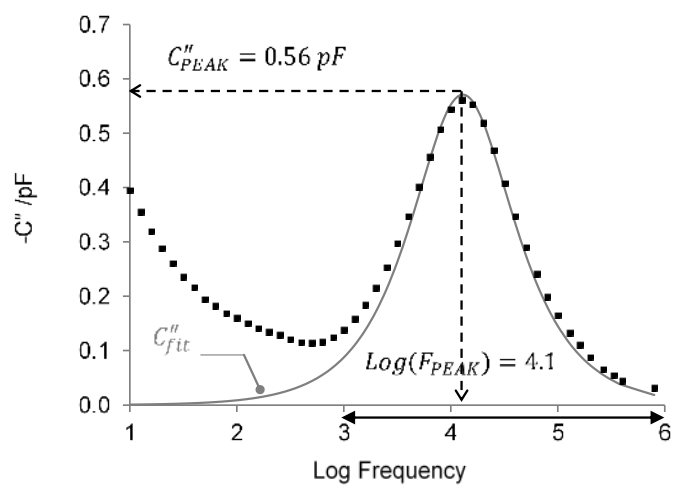

b

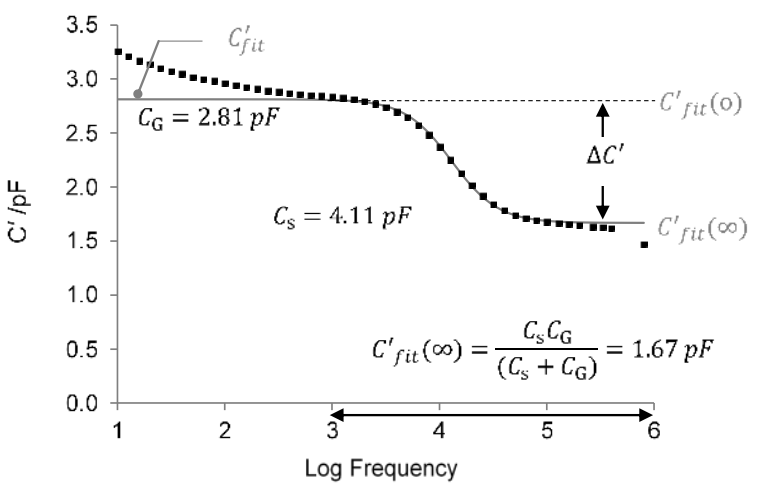

Selected frequency range for fitting: $1 \mathrm{kHz}$ to $1 \mathrm{MHz}$

Fig. 8 A typical capacitance spectrum of $7 \mathrm{~g}$ double distilled water in a $10 \mathrm{~mL}$ Type I glass tubing vial (Adelphi VC010-20C) which has been modified with a pair of external copper electrodes of dimensions $10 \times 19 \mathrm{~mm}$ and positioned $3 \mathrm{~mm}$ from the base of the vial. The temperature of the liquid is $22{ }^{\circ} \mathrm{C}$. The line (grey line) shows the fit to the equivalent circuit model shown in Fig. 6 and the symbols (black dot) show the data points. The horizontal line with double headed arrow marks the frequency range for fitting. The disagreement between the fit and the data at the lower frequencies is due to the presence of a separate dielectric process which is thought to arise from Maxwell-Wagner (space charge) polarization within the glass wall of the vial

Using expressions Eq. 8 and Eq. 9 it is possible to rationalize the changes in both $F_{P E A K}$ and $C_{P E A K}^{\prime \prime}$ as the liquid within the vial is cooled to lower temperatures:

The parameter $F_{P E A K}$ depends on the inverse of both the solution resistance and the sum of the solution capacitance and the glass wall capacitance. However, given the much greater temperature coefficient of electrical resistance over electrical capacitance then $F_{P E A K}$ is particularly sensitive to the temperature of the product via changes in the solution resistance. 
The magnitude of the peak $\left(C_{P E A K}^{\prime \prime}\right)$ is a function of the height of the liquid layer within the region bounded by the electrodes and in intimate contact with the inside of the vial. Figure 9 shows the relationship between $C_{P E A K}^{\prime \prime}$ and the fill volume/height of the liquid in the TVIS vial. The dependence of $C_{P E A K}^{\prime \prime}$ on the height of liquid in the region bounded by the electrodes is only quasi-linear in the mid band of this region. For this vial, of internal diameter $22.05 \mathrm{~mm}$, the linear region starts at $\sim 2 \mathrm{~mm}$ above the bottom edge of the electrode and finishes approximately $2 \mathrm{~mm}$ from the top edge of the electrode.

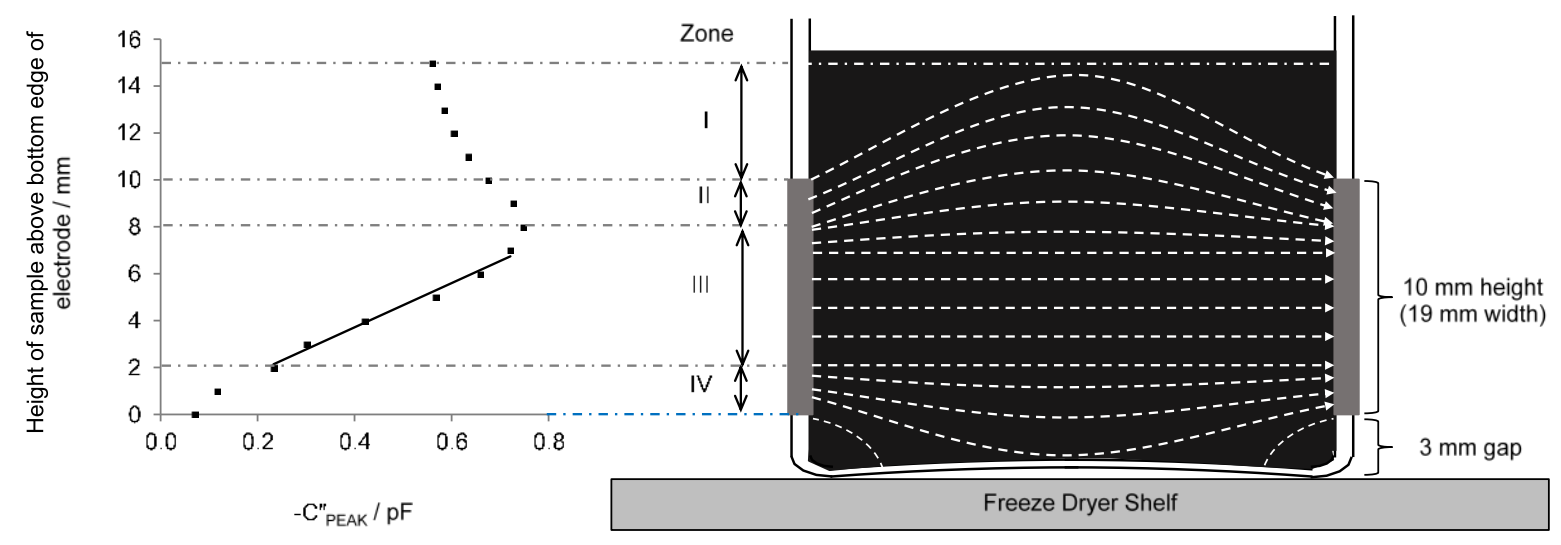

Fig. 9 The dependence of $C_{P E A K}^{\prime \prime}$ on the height of liquid water from bottom edge of the electrode. Zone $I$ is the fringing field region above the electrode which continues to contributes to the overall capacitance despite the fact that the liquid in this region is above the top edge of the electrode; Zone II is the non-linear response region to the liquid at the top of the volume of liquid bounded by the electrodes; Zone III is the quasi-linear response region to the liquid in the region bounded by the electrodes; Zone $I V$ is the non-linear response region to the liquid at the top of the bottom of liquid bounded by the electrodes.

Another way of expressing the height of the sample within the volume bounded by the external electrodes (i.e. the electrode space) is to state this quantity in relative terms, in the format of a fill factor $(\varnothing)$ which is ratio of the height of the liquid in the electrode space to the height of the electrode. Table 3 shows the volumes of liquid water at $20{ }^{\circ} \mathrm{C}$ which provide fill factors of 0 to 1.2 .

Table 3 Fill volumes $\left(20^{\circ} \mathrm{C}\right)$ required to deliver fill factors from $\emptyset=0$ (the liquid fill to the bottom of the electrode) to $\varnothing=1.2$ (the maximum volume of liquid that can be measured by the TVIS system, i.e. the volume of liquid within the field lines propagated by the external electrodes). Data is for the Adelphi VC010-20C vial.

\begin{tabular}{|c|c|c|c|c|c|}
\hline$\varnothing$ & Volume $(\mathrm{mL})$ & $\varnothing$ & Volume $(\mathrm{mL})$ & $\varnothing$ & Volume $(\mathrm{mL})$ \\
\hline 0 & 0.5 & 0.5 & 2.60 & 0.9 & 4.28 \\
\hline 0.2 & 1.34 & 0.6 & 3.02 & 1.0 & 4.70 \\
\hline 0.3 & 1.76 & 0.7 & 3.44 & 1.1 & 5.12 \\
\hline 0.4 & 2.18 & 0.8 & 3.86 & 1.2 & 5.54 \\
\hline
\end{tabular}

Note that on freezing the fill factor will increase given that the density of ice is lower than that of liquid water. The density of water is $0.99819 \mathrm{~g} \cdot \mathrm{cm}^{-3}\left(20^{\circ} \mathrm{C}\right)$ and the density of ice at a range of sub-zero temperature can be estimated from $\rho=917-0.13 \times \mathrm{T}\left(\mathrm{kg} \cdot \mathrm{m}^{-3}\right)$, where $\mathrm{T}$ is given in ${ }^{\circ} \mathrm{C}$ (Melinder 2010). For example $\rho($ ice $)=0.9194$ $\mathrm{g} \cdot \mathrm{cm}^{-3}$ at $-20^{\circ} \mathrm{C}$ such that a liquid fill factor of 0.7 at $20^{\circ} \mathrm{C}$ will increase to 0.76 . 


\subsubsection{Enhanced model for the TVIS Spectrum of the Liquid State}

The basic model, which assumes that the glass wall behaves like a simple capacitor, with a frequency independent response, does not adequately account for the dielectric response of the glass wall towards the lower frequencies of the TVIS spectrum which, rather than having a flat frequency response, is characterized by an increase in both the real and imaginary impedance (For example, see the real part capacitance spectra at frequencies below $1 \mathrm{kHz}$ or $\log$ Frequency $=3$, Fig. 8b). This feature of the low frequency response (which is observed for many micro-structured materials containing semimobile charge carriers) is often referred to as an anomalous low frequency dispersion (abbreviated to LFD in the dielectric literature) and in the case of glass results from proton percolation (space charge polarization) within the porous/hydrated silica. Because the extent of charge migration (i.e. the numbers of charges and the length of the percolation path) is dependent on time (and hence frequency of the applied field) then the magnitude of the induced polarization also increases as the frequency of the applied field decreases, resulting in the ever increasing contribution to the glass wall capacitance and dielectric loss. ${ }^{3}$ This percolation of protonic charges, through the glass wall microstructure, provides an additional admittance to that associated with the atomic and electronic polarizations that define the instantaneous capacitance of the glass. In the impedance community this low frequency dispersion is often modeled using another circuit element, known as a constant phase element $(C P E)$ which, given the additionality of its admittance, is positioned in parallel with the instantaneous capacitance of the glass wall $\left(C_{G}\right)$.

The admittance $(Y)$ and hence impedance $(Z)$ of a constant phase element $(C P E)$ is given by

$$
Y_{\mathrm{CPE}}=\frac{1}{Z_{\mathrm{CPE}}}=Q_{o}(i \omega)^{p}
$$

where $Q_{o}$ has the numerical value of the admittance at the angular frequency of $\omega=1 \mathrm{rad} \cdot \mathrm{s}^{-1}$, and units of $S \cdot s^{p}$, and $p$ defines the impedance phase angle $(\vartheta)$ according to the expression $\vartheta=$ $-(90 \times p)$ degrees. If the object behaves more like a capacitor then $p$ tends to 1 (for a pure capacitor, $p=1$ hence $1 / Z_{\mathrm{C}}=Y_{\mathrm{C}}=Q_{o}(i \omega)^{1}=i \omega C$ and $\vartheta=-90^{\circ}$ ) whereas if the object behaves more like a resistor then $p$ tends to 0 (for a pure resistor $p=0$ hence $1 / Z_{\mathrm{R}}=Y_{\mathrm{R}}=Q_{o}(i \omega)^{0}=1 / R$ and $\vartheta=0^{\circ}$ ). The Nyquist plot of the impedance spectrum (where $-Z^{\prime \prime}$ is plotted against $Z^{\prime}$ ) and the Cole-Cole plot of the capacitance spectrum (where $-C^{\prime \prime}$ is plotted against $C^{\prime}$ ) provide useful ways to display the characteristics of this low frequency dispersion (Fig. 10). For the $C P E_{\mathrm{G}}$ alone, i.e. without the instantaneous capacitance of the glass wall, one can see how the increase in the negative of the imaginary impedance, $-Z^{\prime \prime}$ towards low frequency is directly proportional to the increase in the real part impedance, $Z^{\prime}$ (Fig. 10a grey line). In other words, the ratio of the two parameters is invariant with frequency, hence the name constant phase element $(C P E)$. The phase angle can be determined from the slope of the impedance phasor against the axis for the real part impedance of the Nyquist plot, according to Eq. 13:

$$
\vartheta=\operatorname{atan}(\text { slope })
$$

\footnotetext{
${ }^{3}$ A more comprehensive treatment of the dielectric properties of glass (porous silica) can be found in the publication of (Ryabov et al. 2001)
} 

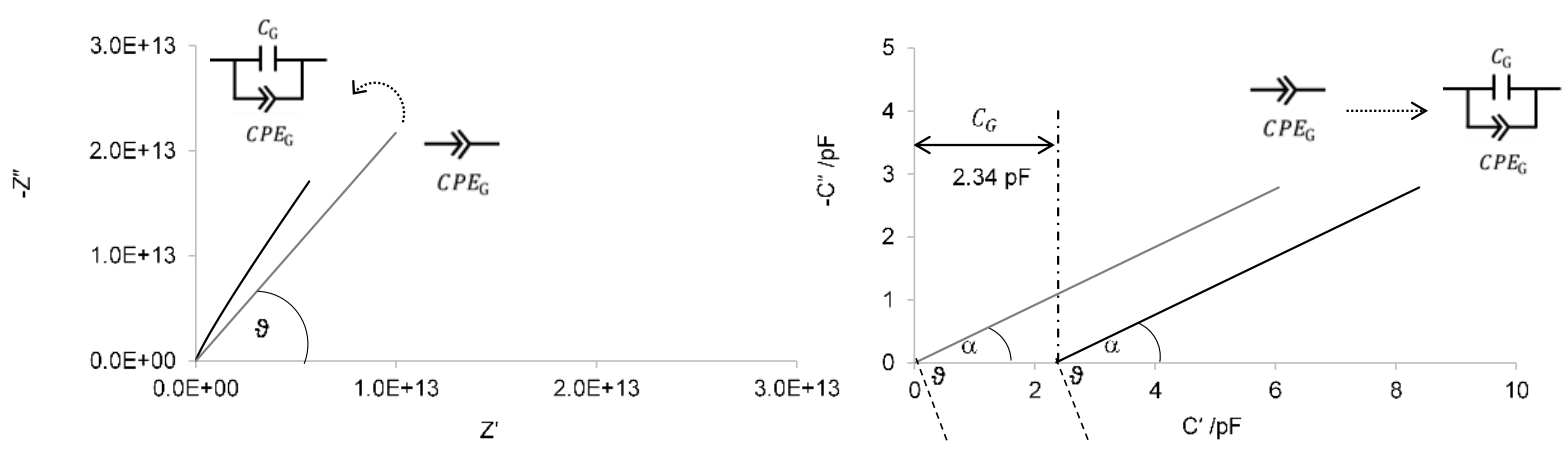

Fig. 10 (a) Nyquist plot of imaginary part impedance vs real part impedance for a $C P E_{\mathrm{G}}$ alone (grey line) and a $C P E_{G}$ in parallel with a capacitor $\left(C_{\mathrm{G}}\right)$ (black line); (b) Cole-Cole plot of imaginary part capacitance vs real part capacitance for the same $C P E_{G}$ (grey line) and the $C P E_{\mathrm{G}}$ in parallel with a capacitor $\left(C_{\mathrm{G}}\right)$ (black line); On the Nyquist plot, for the $C P E_{\mathrm{G}}$ alone (grey line) the phase angle, in radians, is given by atan (slope). On the Cole-Cole plot the phase angle for the $C P E_{\mathrm{G}}$ (in radians) is given $\pi / 2-$ atan (slope), irrespective of whether the model contains the additional capacitance element or not. Each data set for this figure has been created from values for a constant phase element $\left(C P E_{\mathrm{G}}\right)$ and capacitor $\left(C_{\mathrm{G}}\right)$ that are typical to those observed from experimental data for liquid water at $20.1^{\circ} \mathrm{C}$ in an Adelphi VC010-20C glass tubing vial (of nominal volume $10 \mathrm{~mL}$ ) and with $10 \times 19 \mathrm{~mm}$ electrodes at a height of $3 \mathrm{~mm}$ from the base of the vial. Those value are $Q_{o}=1.587 \times 10^{-12} S \cdot s^{p}$ and $p$ $=0.725$ for the magnitude and phase of the glass wall $C P E_{\mathrm{G}}$ and $C_{\mathrm{G}}=2.34 \mathrm{pF}$ for the glass wall capacitance. The frequency range over which the impedance has been simulated is $0.001 \mathrm{~Hz}$ to $1 \mathrm{MHz}$

On the Cole-Cole plot (Fig. 10b) the angle $(\alpha)$ between the capacitance scalar and the axis for real part capacitance $\left(C^{\prime}\right)$ provides an alternative assessment of the impedance phase angle (where $\alpha=$ $90-\vartheta)$.

In this example (Fig. 10a) the slope of the impedance vector with the axis for real part impedance is 2.173 (note that $-Z^{\prime \prime}$ is plotted against $Z^{\prime}$ so the slope is positive). It follows that $\vartheta=$ $1.1395 \mathrm{rad}$ or $65.29^{\circ}$ and therefore $p=0.725$ (from $p=\vartheta / 90$ ).

When the capacitor is added in parallel (Fig. 10a black line) then the line for the impedance magnitude on the Nyquist curve appears to have a steeper gradient, and when examined more closely is in fact slightly curved. Therefore, it is not possible to estimate the phase angle of a constant phase element directly from the Nyquist plot when there is a capacitor in parallel. Fortunately, the impact of the additional capacitance $\left(C_{\mathrm{G}}\right)$ on the Cole-Cole plot (Fig. 10b) is more straight forward, given that $C_{\mathrm{G}}$ only has a real part and that capacitances in parallel can be added together to get the total capacitance. As a consequence, the net effect of the $C_{\mathrm{G}}$ on the Cole-Cole plot is to shift the complex capacitance along the real part axes by an amount which is equal to the value of $C_{\mathrm{G}}$. The phase angle may still be determined from the slope of the capacitance vector with the axis for the real part capacitance.

The Bode plot of the imaginary vs real parts of the impedance (Fig. 11) provides a convenient way to predict the magnitude of the constant phase element, $Q_{o}$, given that it has a numerical value of $1 /|Z|$ at an angular frequency of $\omega=1 \mathrm{rad} \mathrm{s}^{-1}$ (or $f=\omega / 2 \pi=0.16 \mathrm{~Hz}$ ). Fig. 11a and Fig. 11b highlight the opportunity to 'read' off the magnitude of the constant phase element at a frequency of $\omega=1$ (or $\log \omega=0$ ). In the example of the $C P E_{\mathrm{G}}$ without $C_{\mathrm{G}}$ (Fig. 11a) $\log |Z|=11.8, Z=630 \mathrm{G} \Omega$ and therefore $Q_{o}=1.587 \times 10^{-12} \mathrm{~S} . \mathrm{s}^{\mathrm{p}}$. When the capacitor is added in parallel (Fig. $11 \mathrm{~b}$ and Fig. $11 \mathrm{~d}$ ) there is a moderate change in the value of $\log |Z|$ at $\log \omega=0$, from $\log |Z|=11.8$ to $\log |Z|=11.4$, i.e. $|Z|$ changes from $630 \mathrm{G} \Omega$ to $251 \mathrm{G} \Omega$. This intercept value is close enough (i.e. within one order of 
magnitude) of the intercept value for $C P E_{\mathrm{G}}$ without $C_{\mathrm{G}}$ such that it provides an acceptable starting parameter estimate when fitting data with a $C P E_{\mathrm{G}}=C_{\mathrm{G}}$ model.

a

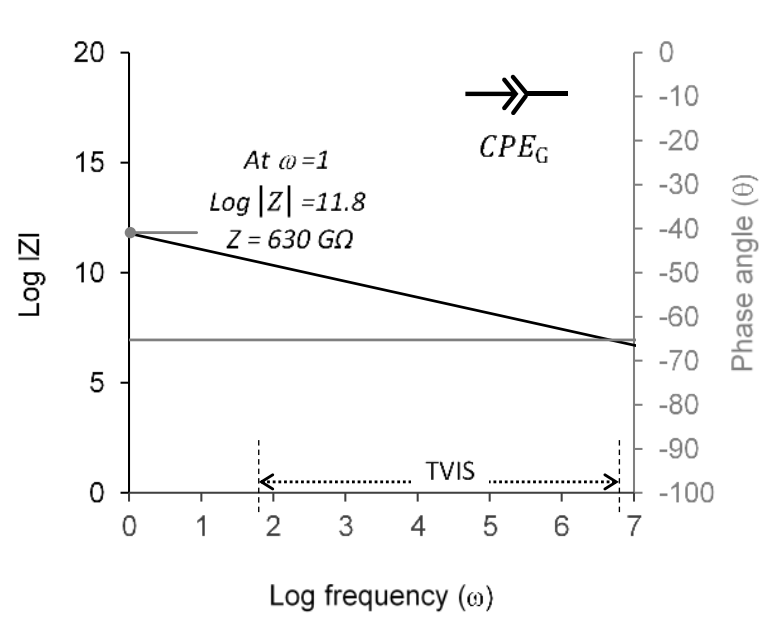

c

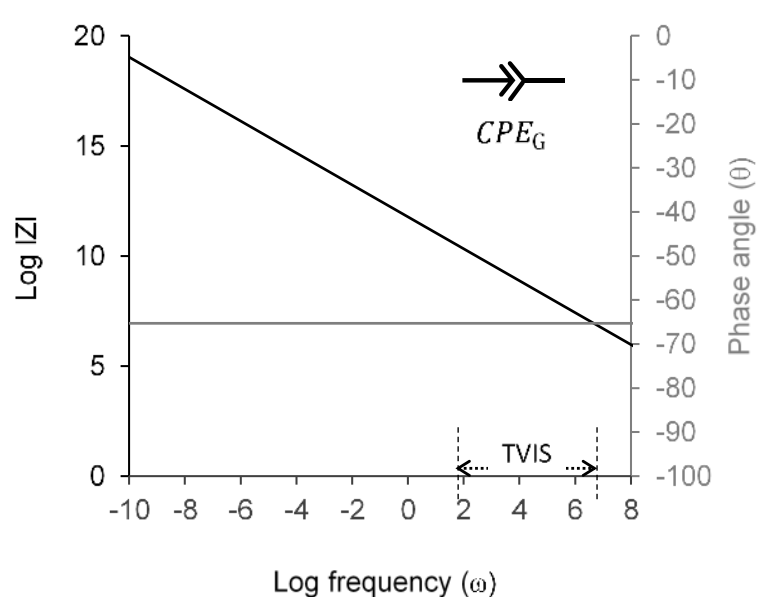

b

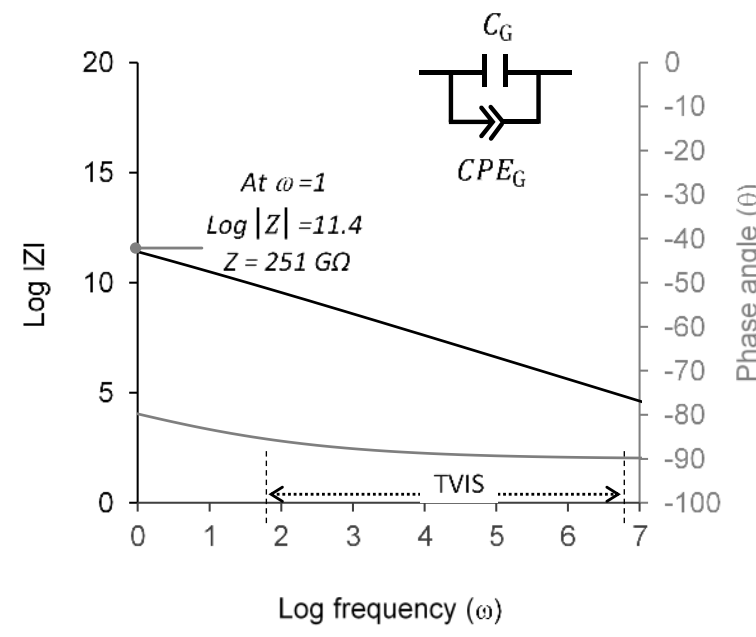

d

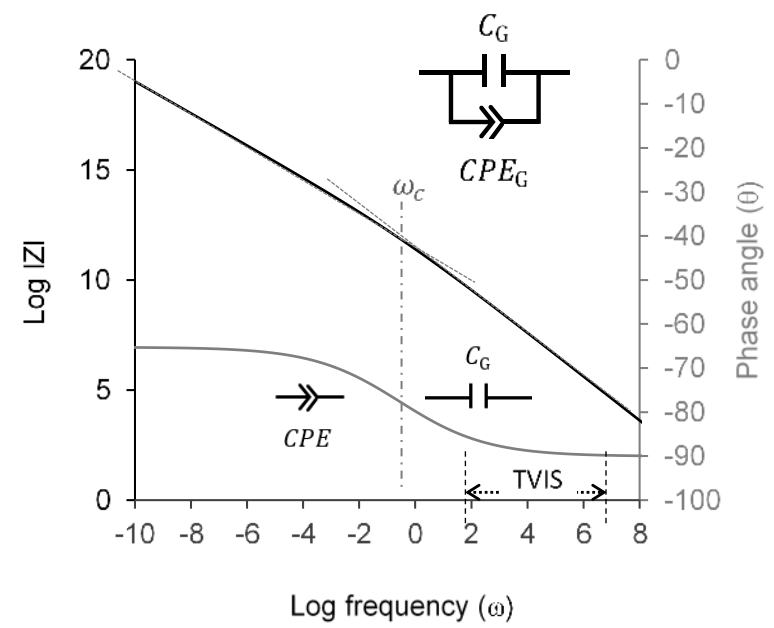

Fig. 11 Bode plot of impedance magnitude and phase angle for a $C P E_{\mathrm{G}}$ alone (a and $\mathbf{c}$ ) and for a $C P E_{\mathrm{G}}$ in parallel with a capacitor $C_{\mathrm{G}}(\mathbf{b}$ and $\mathbf{d})$. The frequency scale on the top graphs have been set to show the range of frequencies which are accessible to the TVIS instrument ( $\log \omega=1.8$ to 6.8 ) while extending the range to $\omega=1$ in order to demonstrate how an estimate for $Q_{o}$ might be derived from the intercept with the y-axis (at $\log \omega=0$, i.e. $\omega=1 \mathrm{rad} \mathrm{s}^{-1}$ or $0.16 \mathrm{~Hz}$ ). The frequency range marked by the two dotted lines immediately above the frequency axis shows the experimental frequency range accessible to the TVIS instrument $(10 \mathrm{~Hz}$ to $1 \mathrm{MHz})$. The frequency range for the bottom two graphs has been extended to ultra-low frequencies in order to 'capture' the dominance of the CPE element $\left(C P E_{\mathrm{G}}\right)$ at those frequencies (well below the frequency range achievable by commercial instrumentation)

The frequency range of the Bode plots in Fig. 11c and Fig. 11d has been extended way below that achievable by any available commercial impedance or broad band dielectric spectrometer, in order to demonstrate the complete frequency response of a parallel combination of a constant phase element and a capacitor. At low frequency $\left(<10^{-2} \mathrm{~Hz}\right)$ the impedance of the $C P E_{\mathrm{G}}$ is lower than that of the capacitor $C_{\mathrm{G}}$ and so the $C P E_{\mathrm{G}}$ begins to dominate the total impedance and hence the phase angle converges on that of $C P E_{\mathrm{G}}$, i.e. $=65.29^{\circ}$. At high frequency $\left(<10^{2} \mathrm{~Hz}\right)$ the impedance of the capacitor is lower than that of the $C P E_{\mathrm{G}}$ element and so the capacitance $C_{\mathrm{G}}$ begins to dominate the impedance 
spectrum and the phase angle therefore converges on a value of $-90^{\circ}$. The transition frequency $\left(\omega_{c}\right)$ between the dominance of one element and the other is marked by the vertical dot-dashed line (Fig. 11d).

This understanding of how to 'read' the Cole-Cole plot to determine the phase angle of a $C P E_{\mathrm{G}}$ element, and the Bode plot to determine the magnitude of the $C P E_{\mathrm{G}}$ element, is helpful when trying to find starting parameters (estimates) for fitting an equivalent circuit model using modelling software such as Zview ${ }^{\circledR}$ or RelaxIS.

The starting parameters for the other elements in the enhanced model $\left(C_{\mathrm{S}}, R_{\mathrm{s}}, C_{\mathrm{G}}\right)$ can be estimated as follows:

1. An estimate for $C_{\mathrm{G}}$ can be 'read' directly from the real part capacitance spectrum at a frequency on the low side of $F_{P E A K}$ (approximately where the spectrum states to level out, before increasing once again towards even lower frequency, where the low frequency dispersion starts to impact the spectrum).

2. An estimate for $C_{\mathrm{s}}$ is determined by 'reading' an estimate for $C^{\prime}(\infty)$ from the real part capacitance spectrum at a frequency on the high side of $F_{P E A K}$, where the spectrum starts to level out, and then substituting the estimate for $C^{\prime}(\infty)$ along with the estimate for $C_{\mathrm{G}}$ from (i) above into Eq. 10.

3. Once $C_{\mathrm{G}}$ and $C_{\mathrm{s}}$ are known then an estimate for $R_{\mathrm{s}}$ can be determined from Eq. 9 where the value for $F_{P E A K}$ is estimated from reading the peak frequency off the dielectric loss spectrum.

Figure 12 shows the result of fitting the enhanced model to the totality of the impedance response from the vial, including the $C P E_{\mathrm{G}}$ element which models the dispersive nature of the glass wall. The graphs on the left show the results from fitting the enhanced model, where $C P E_{\mathrm{G}}=C_{\mathrm{G}}$ represents the glass wall impedance and $C_{\mathrm{S}}=R_{\mathrm{S}}$ represents the sample impedance (The symbol '=' means two elements in parallel). The graphs on the right show the contribution to the enhanced model from the elements included in the simplified model, i.e. $C_{\mathrm{G}}-C_{\mathrm{S}}=R_{\mathrm{s}}$. These graphs have been created by a process involving the fit to the enhanced model, followed by the exclusion of the CPE element and running a simulation of the reduced model but keeping the same parameters that were determined when fitting the enhanced model.

It is clear from Fig. 12b that the simulated response, in the absence of the $C P E$ element does not perfectly match the data on the low frequency side of the Maxwell-Wagner peak and that the influence of the low frequency dispersive characteristics of the glass extend to frequencies as high as $10 \mathrm{kHz}$. 

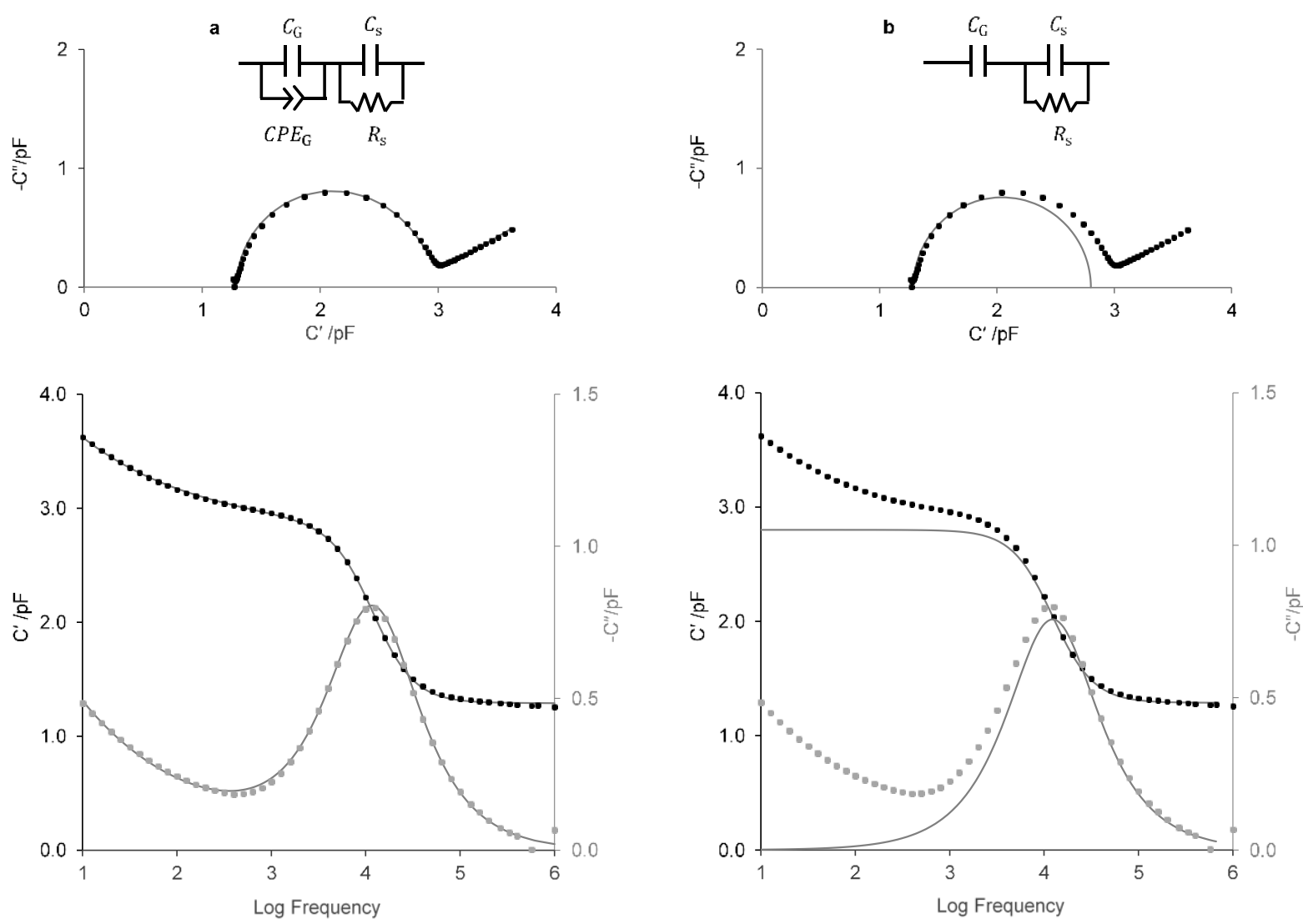

Fig. 12 Enhanced model for the MW process of a Adelphi VC010-20C glass tubing vial with $10 \times 19$ $\mathrm{mm}$ electrodes at a distance of $3 \mathrm{~mm}$ from the base of the vial, containing $3.4 \mathrm{~g}$ of ultrapure water $(\varnothing=$ 0.7 ) . The temperature of the water is $\sim 22{ }^{\circ} \mathrm{C}$. (a) shows the enhanced model which includes a $C P E_{\mathrm{G}}$ for the low frequency response associated with the glass wall impedance whereas (b) has the $C P E_{\mathrm{G}}$ removed and the impedance response from the other elements simulated. The fit parameters determined by the software are $Q_{o}=3.94 \times 10^{-12} S \cdot s^{p}, p=0.658, C_{\mathrm{G}}=2.34 \mathrm{pF}, R_{\mathrm{S}}=2.49 \mathrm{M} \Omega, C_{\mathrm{s}}=2.38 \mathrm{pF}$

\subsubsection{Cell Constant Determination}

An alternative approach to determining the geometric cell constant of the sample space (Eq. 3), which is ideally suited to a parallel plate capacitor equation) is to express the cell constant in terms of the empty cell capacitance $\left(C_{\mathrm{o}}\right)$. A convenient experimental approach for determining this parameter is to plot values the sample capacitance $C_{\mathrm{S}}$, as a function of the dielectric constant of a range of polar liquids with sufficient conductivity to give relaxation to a MW process and then the empty cell capacitance $\left(C_{\mathrm{o}}\right)$ can be estimated straight from the slope of the graph, according to Eq. 14:

$$
C_{\mathrm{s}}=k \varepsilon_{\mathrm{o}} \varepsilon_{\mathrm{s}}=C_{\mathrm{o}} \varepsilon_{\mathrm{s}}
$$

Values for $C_{\mathrm{s}}$ are taken from fitting the enhanced model to the complex capacitance spectrum for each liquid at a fill height in excess of $15 \mathrm{~mm}$ from the internal base of vial (in the case of an Adelphi VC010-20C vial with a single electrode pair of dimensions $10 \times 19 \mathrm{~mm}(\mathrm{~h} \mathrm{x} \mathrm{w})$ and positioned at $3 \mathrm{~mm}$ from the base of vial). At this fill height, the electric field lines between electrodes are fully contained within the liquid volume and therefore give the maximum value of capacitance (Fig. 9). Figure 13 shows the plot of $C_{\mathrm{s}}$ against dielectric constant of various samples (i.e. water, methanol and acetone) at room temperature. The slope from this plot is 0.0525 and the units are in $\mathrm{pF}$, given that the dielectric constant is a dimensionless parameter. 


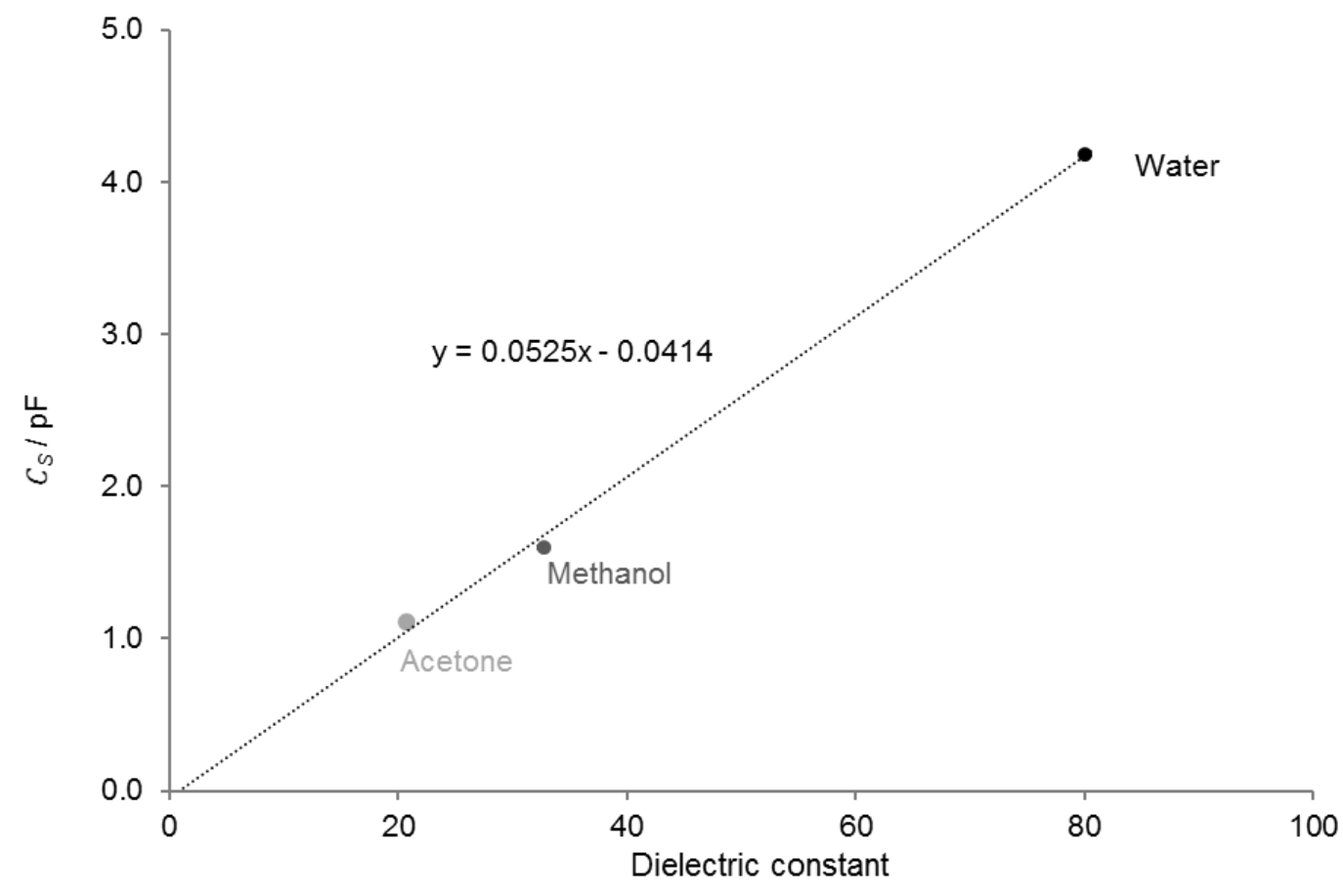

Fig. 13 The sample capacitance as the function of dielectric constant. The slope of the line of best fit is $0.0525 \mathrm{pF}$ and represents the cell constant. Note that this capacitance is lower than the measured capacitance of the TVIS vial when empty (i.e. $0.07 \mathrm{pF}$, Fig. 5) suggesting that there is a small stray capacitance of the order of $0.02 \mathrm{pF}$. Theoretically, one would expect this stray capacitance to show as the value of the intercept on the y-axis. However, the uncertainty in the measured values for the three solvents has probably resulted in some uncertainty in the intercept value such that the stray capacitance is not quantifiable by that approach

Once the empty cell capacitance $\left(C_{o}\right)$ is known, then it is straight forward to estimate the sample capacitance for any other liquid or indeed solid, provided one has an estimate for dielectric constant for that material. This is exploited later in the case of measurements on ice; for example if the permittivity of ice at $-40{ }^{\circ} \mathrm{C}$ is 111.4 , then one expects a sample capacitance, $C_{S}\left(\right.$ ice $\left._{-40^{\circ} \mathrm{C}}\right)$, to be $5.81 \mathrm{pF}$.

Note that the corresponding values for $C_{\mathrm{G}}$ lie within the range $2.98-3.07 \mathrm{pF} ;\left(C_{\mathrm{G}}(\right.$ water $)=3.07$ $\mathrm{pF}, C_{\mathrm{G}}($ methanol $)=2.98 \mathrm{pF}$ and $C_{\mathrm{G}}($ acetone $\left.)=3.03 \mathrm{pF}\right)$. The fact that these values are all lower than the theoretical value $C_{\mathrm{a}-\mathrm{G}}$ of $3.99 \mathrm{pF}$ which has taken into account the additional capacitance of the adhesive (Fig. 7 and Eq. 7) is most likely due to the presence the capacitance associated with the glasssample interface. This capacitance in series with the glass and adhesive capacitance will inevitably reduce the effective capacitance of the glass wall by an amount which appears to depend on the specific nature of the liquid-glass interaction.

It is clear from what has been said here about the characteristics of the composite capacitance (Fig. 14) that its impedance response is somewhat complex and difficult to predict due to the limited understanding of the individual layers that make up this complex impedance, especially the uncertainty in our understanding of the glass-sample interface and the dependency on the nature of the contact with the fill material. This issue will be discussed later in the chapter when a method for determining the interfacial capacitance from measurements on ice, at different temperatures, is explored. 

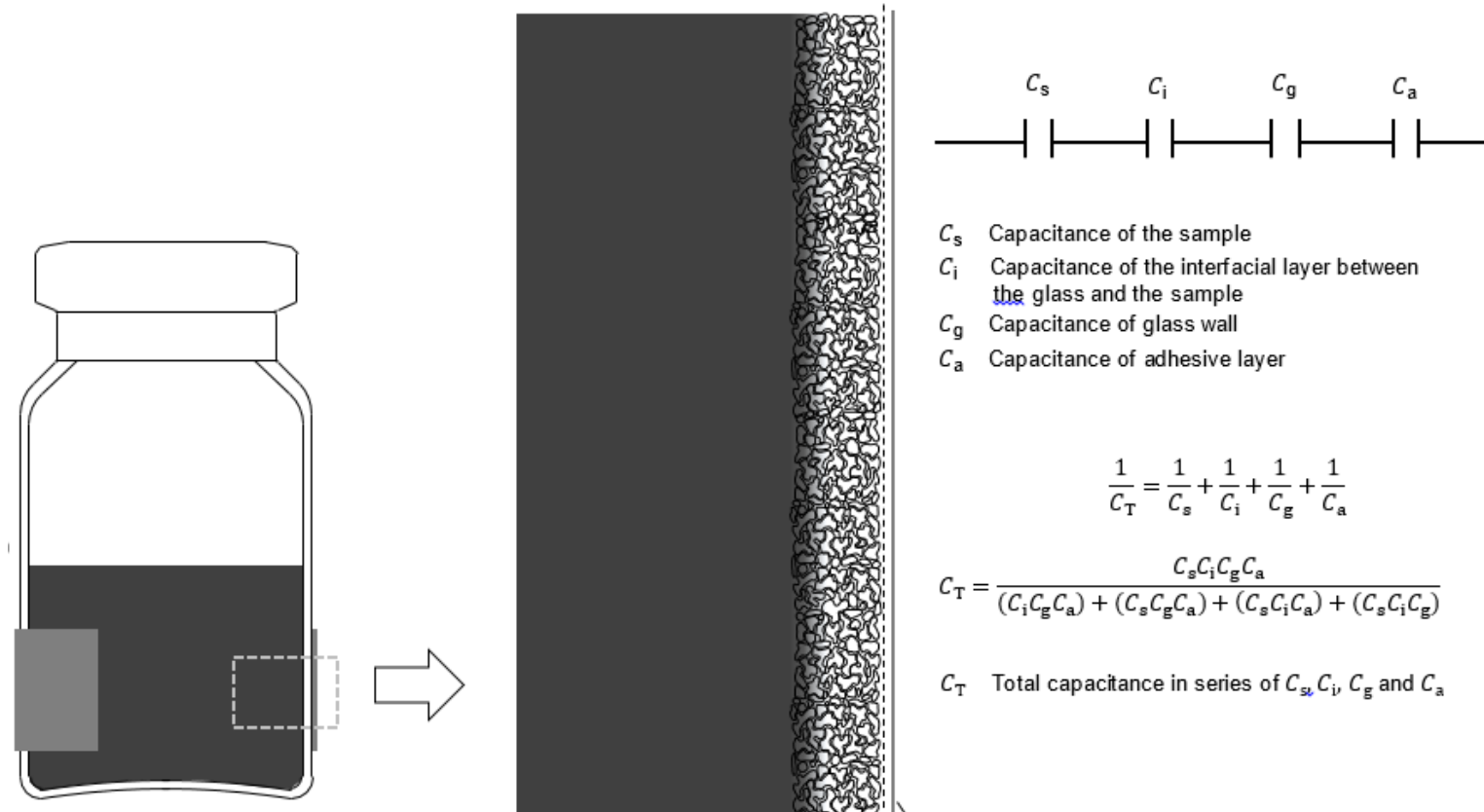

Fig. 14 Illustration showing a close up schematic of the composite capacitance resulting from a layer of adhesive $\left(C_{a}\right)$ in contact with the glass wall of the vial $\left(C_{g}\right)$ which has a microstructure that supports a capacitance $\left(C_{i}\right)$ of an interfacial layer of hydrated glass in contact with the bulk liquid $\left(C_{s}\right)$

\subsubsection{Frozen Water and the Dielectric Relaxation of Ice}

As soon as the water in the vial begins to freeze then the dielectric loss peak moves to lower frequencies. Figure 15 shows the capacitance spectrum of a TVIS-modified Adelphi VC010-20C glass tubing vial (with an electrode pair of dimensions $10 \times 19 \mathrm{~mm}$ ) containing double distilled water, at three temperatures of $+20,-20$ and $-40^{\circ} \mathrm{C}$. The figure has been created partly from experimental data (solid line) and partly from the anticipated response in the microwave range, and beyond, in order to include the dielectric relaxation of water (dotted line). Note that the shape of the dielectric loss peak in the frozen state is rather like that one observes for the MW process in the liquid state and so one might think that it is simply the Maxwell-Wagner process but shifted to lower frequencies because of an increase in the resistance of the sample. However, this observation is not because the electrical resistance of ice is higher than that of water (although that in itself would be expected to shift the peak to lower frequencies) but rather because of the appearance of a new process which is fundamentally different to MW polarization, namely the dielectric relaxation of ice. The dielectric relaxation of ice means that the sample capacitance $\left(C_{\mathrm{s}}\right)$ is no longer constant over the experimental frequency range of the TVIS instrument but has taken on a frequency dependence, whereby the permittivity of the frozen water changes from a value between 120 and 97 at low frequency (which is known as the static relative permittivity $\left(\varepsilon_{s}\right)$ of ice) ${ }^{4}$ to a value between 3.03 and 3.09 at high frequency (which is known as the instantaneous relative permittivity $\varepsilon_{\infty}$ ) (Johari and Whalley 1981). The values given for $\varepsilon_{S}$ and $\varepsilon_{\infty}$ are

\footnotetext{
4 'Static' refers to the low frequency value of capacitance at which all polarization mechanisms have had sufficient time to respond fully to the applied field.
} 
for the limits of temperature $\sim-70{ }^{\circ} \mathrm{C}$ to $0{ }^{\circ} \mathrm{C}$ that are most relevant to freeze-drying applications. There are also changes in the width of the dielectric relaxation for ice, with the dielectric loss peak becoming broader at the lower temperatures of $-40{ }^{\circ} \mathrm{C}$, whereas the width at $-20{ }^{\circ} \mathrm{C}$ is similar to that for the Maxwell-Wagner process of the liquid state.

Dielectric relaxation is a phenomenon which is manifest in the frequency response of the dielectric permittivity, and the dielectric loss, or in the decay of the electric field inside a dielectric on removal of the external field. It results from the change in polarization state of a dielectric component in the system. For example, the water molecule has a permanent dipole which is highly mobile in the liquid state, with its polarization being characterized by a time constant of the order of 9 picoseconds (at $20{ }^{\circ} \mathrm{C}$ ). This translates to: (1) a contribution to the dielectric permittivity at low frequencies when the dipoles have sufficient time to follow the changing polarity of the external field, which stores energy from the external field, (2) a loss of that contribution and reduction in the dielectric permittivity as the dipoles relaxation to a randomized state as the frequency of the applied field exceeds the response time for the dipolar system, and (3) a peak in the dielectric loss during the transition between the ordered/polarized state and the relaxed state as the dipoles absorb energy from the field rather than store it.

The relaxation of liquid water at $20^{\circ} \mathrm{C}$ is centred in the microwave range, close to $18 \mathrm{GHz}$ and way above the experimental range of the TVIS instrument; hence the use of a dotted line on Fig. 15 to indicate that the liquid water relaxation is just a simulation of the theoretical response one might observe if it were possible to extend the frequency range of the TVIS instrument to these high frequencies. And so far as TVIS measurements are concerned, the dielectric permittivity of liquid water can be considered to be constant over the frequency range of the instrument, which then justifies the choice of a simple capacitor to model the liquid state of water. However, in the frozen state, water molecules form a regular architecture with oxygen atoms positioned in a repeating tetrahedral arrangement and with the hydrogen atoms randomly arranged within the crystal according to the Bernal-Fowler-Pauling rules (Hobbs 1974, Bernal and Fowler 1933, Pauling et al. 1935) such that the rotational motion of a water molecule is significantly restricted, in ice, relative to the degrees of freedom that water molecules have in the liquid state. As a result the experimental relaxation time is reduced by six orders of magnitude. For example, the relaxation time of ice is $162 \mu \mathrm{s}$ (at $-20^{\circ} \mathrm{C}$ ) which translates to a relaxation peak centred on $\sim 1 \mathrm{kHz}$ (in contrast to $\sim 18 \mathrm{GHz}$ at $20^{\circ} \mathrm{C}$ ). At the limits of the temperatures encountered in a freezedrying process (e.g. $-70{ }^{\circ} \mathrm{C}$ ) this relaxation frequency is expected to be low as $100 \mathrm{~Hz}$. However, that frequency is well within the experimental frequency range of the TVIS instrument. 

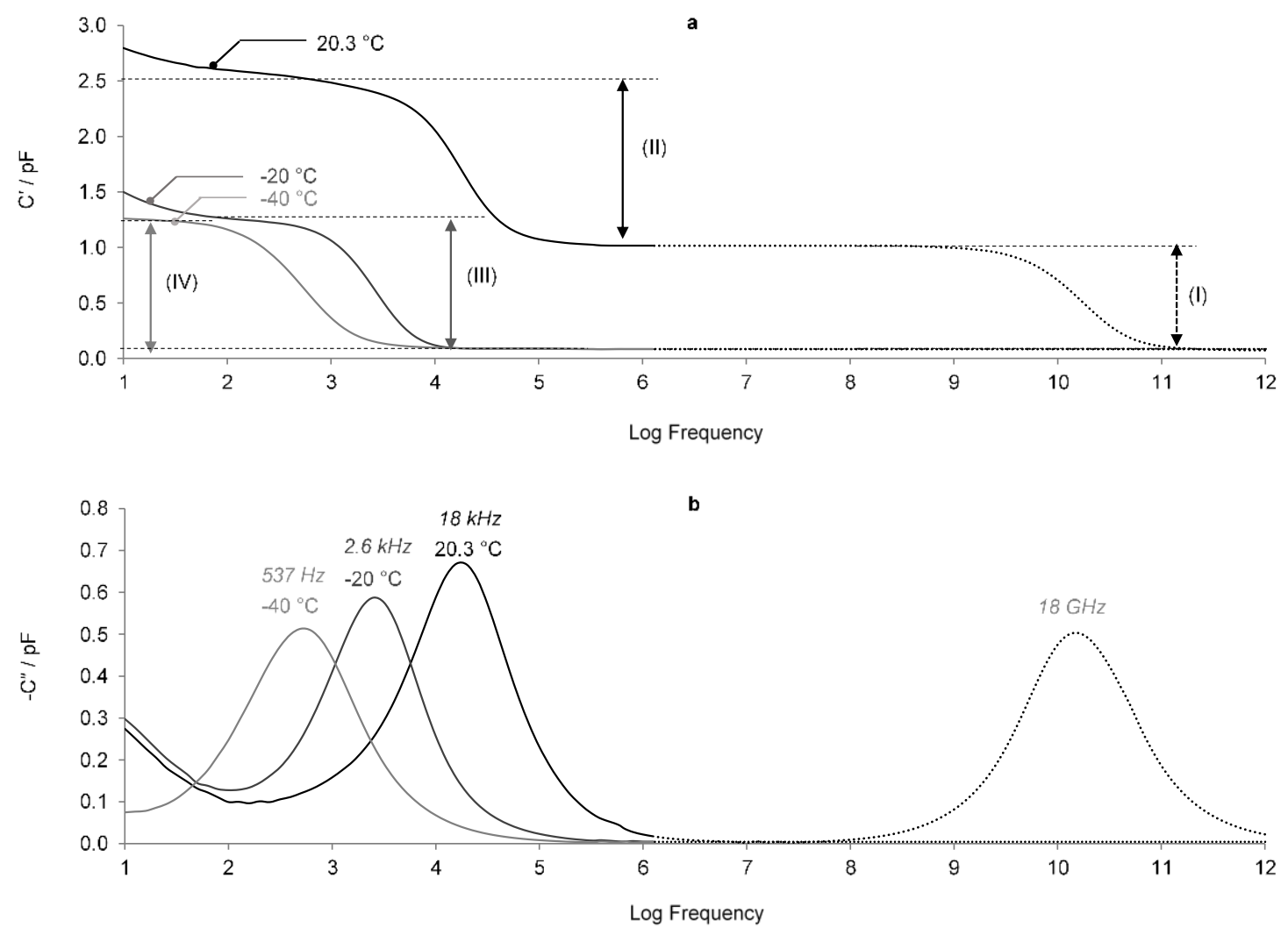

Fig. 15 Part simulation and part real data for double distilled water in a TVIS-modified Adelphi VC010-20C glass tubing vial (with electrodes of dimension $10 \times 19 \mathrm{~mm}$ and positioned $3 \mathrm{~mm}$ from the base). The real (a) and imaginary (b) parts of capacitance at three temperatures of $+20,-20$ and $-40{ }^{\circ} \mathrm{C}$ are shown. Approximate increments in the real part capacitance spectrum (a) for each temperature are marked with Roman numerals: $(I)$ is the simulated contribution to the capacitance resulting from polarization of the water dipole in liquid water, $+20^{\circ} \mathrm{C}$, with a loss peak frequency of $\sim 18 \mathrm{GHz}$; (II) is the contribution to the measured capacitance resulting from the Maxwell-Wagner polarization of the glass wall of the TVIS vial, $+20{ }^{\circ} \mathrm{C}$, with a loss peak frequency of $17.8 \mathrm{kHz}$; (III) and (IV) are the contribution to the measured capacitance resulting from dielectric polarization of ice at $-20^{\circ} \mathrm{C}$ and -40 ${ }^{\circ} \mathrm{C}$ respectively, for which the dielectric loss peak frequencies are $2.57 \mathrm{kHz}$ and $537 \mathrm{~Hz}$

The dielectric properties of ice have been studied in some detail for well over sixty years. However, the most accurate investigation of ice Ih (for both $\mathrm{D}_{2} \mathrm{O}$ and $\mathrm{H}_{2} \mathrm{O}$ ) was reported by Johari and Whalley (1981) with ice Ih ice being revisited later by (Shinyashiki et al. 2009). From these works, the main features of the dielectric relaxation of ice can be summarised as follows (Popov et al. 2015): (1) the relaxation is characterised by a single relaxation time at temperatures above $250 \mathrm{~K}$ (which can be described by a Debye function, Eq. 15) but there is a symmetrical broadening of the peak as the temperature is lowered below $250 \mathrm{~K}$ (which can be described by a Cole-Cole function, Eq. 16); (2) the high frequency limit of the dielectric permittivity $\left(\varepsilon_{\infty}\right)$ is almost independent of temperature, whereas the static permittivity $\left(\varepsilon_{s}\right)$ obeys the Curie-Weiss law $\left.\varepsilon_{s}-\varepsilon_{\infty}\right)=\mathrm{A} /\left(\mathrm{T}-\mathrm{T}_{\mathrm{CW}}\right)$, where $\mathrm{T}_{\mathrm{CW}}$ tends to zero (Johari and Whalley 1981); (3) The temperature dependencies of the relaxation times $(\tau)$ above and below $250 \mathrm{~K}$, both obey the Arrhenius law with activation energies of $\sim 53 \mathrm{~kJ} \mathrm{~mol}^{-1}$ and $\sim 19 \mathrm{~kJ} \mathrm{~mol}^{-1}$, respectively. 


$$
\begin{gathered}
\varepsilon^{*}(\omega)=\frac{\left(\varepsilon_{s}-\varepsilon_{\infty}\right)}{(1+i \omega \tau)}+\varepsilon_{\infty} \\
\varepsilon^{*}(\omega)=\frac{\left(\varepsilon_{s}-\varepsilon_{\infty}\right)}{\left(1+(i \omega \tau)^{1-\alpha}\right)}+\varepsilon_{\infty}
\end{gathered}
$$

$\alpha$ is an exponent which models the width of the relaxation peak. When $\alpha<1$ the relaxation is said to be stretched; In other words it extends over a wider range of frequencies than a Debye relaxation. When $\alpha=0$ the Cole-Cole model (Eq. 16) reduces to the Debye model (Eq. 15). For example, at the temperatures of $-20{ }^{\circ} \mathrm{C}$ and $-40{ }^{\circ} \mathrm{C}$ the Cole-Cole parameter changes from 1.00 to 0.97

Only recently has a definitive, mechanistic model emerged which begins to explain the features of dielectric broadening in terms of a transition from one charge propagation mechanism, to another (Popov et al. 2017), i.e. orientation charge propagation transferring L-D defects through the ice matrix to ionic charge propagation of $\mathrm{H}_{3} \mathrm{O}^{+}$and $\mathrm{OH}^{-}$ion pairs, whereby protons hop through the like matrix in a similar manner to the Grotthus mechanism. This is interesting from a freeze-drying point of view because it potentially provides a mechanism by which changes in ice crystal structure might be elucidated.

\subsubsection{Model for the TVIS spectrum of frozen water}

In the literature, the majority of dielectric spectroscopy measurements of ice have been undertaken using parallel plate electrodes made of metal (invariably gold-coated) and appropriate models developed for the overall measured response which incorporate the impedance of the electrodesample interface (namely electrode polarization). In the case of the TVIS vial, the interfacial properties of the system are defined by the glass wall-sample impedance which, in a basic equivalent circuit model, may be approximated by a simple capacitance $\left(C_{G}\right)$. In reality, the glass will retain the constant phase element behavior that was described by the enhanced model for the TVIS vial containing liquid sample (where the $C P E_{\mathrm{G}}$ models the fractal polarization mechanism associated with the percolation of protonic charge) (Fig. 12a).

In the new model for frozen water (ice) the sample capacitance $\left(C_{s}\right)$ is no longer constant across the frequency range of the instrument $(10 \mathrm{~Hz}$ to $1 \mathrm{MHz})$ but undergoes a step-like change in the real part capacitance and a peak in the imaginary capacitance due to the dielectric relaxation of ice. The sample capacitance, $C_{\mathrm{s}}$, (which in the liquid state is based on the assumption that the dielectric properties of the liquid are independent of frequency, i.e. $C_{\mathrm{s}}$ was the static capacitance of the sample) is now replaced by a distributed element $\left(D E_{\mathrm{s}}\right)$ in parallel with an instantaneous capacitance $C_{\mathrm{s}}(\infty)$. The distributed element $\left(D E_{S}\right)$ represents the dispersive part of the Cole-Cole function (which models the dielectric relaxation of ice) whereas the instantaneous capacitance $C_{\mathrm{s}}(\infty)$ represents the very fast induced polarization processes associated with electronic and atomic charge distortions in ice. The sample resistance $\left(R_{\mathrm{S}}\right)$ is presumed to be so high that it can be ignored, i.e. removed from the model (Fig. 16). The frequency dependence of the sample capacitance, $C_{\mathrm{s}}(f)$, which is due to the dielectric relaxation of ice, results in the sample capacitance changing from a value of $C_{\mathrm{s}}(0)$ at low frequency (the static capacitance) to a value of $C_{\mathrm{s}}(\infty)$ at high frequency (the instantaneous capacitance). As a consequence, the capacitance of the model, $C_{f i t}^{\prime}(f)$, is also frequency dependent changing from a value of $C_{f i t}^{\prime}(0)$ at low frequency to a value of $C_{f i t}^{\prime}(\infty)$ at high frequency.

In the example fit to the TVIS spectrum of frozen water at $-20^{\circ} \mathrm{C}$ and $-40{ }^{\circ} \mathrm{C}$ (Fig. 17) the low frequency data, from where the $C P E_{\mathrm{G}}$ element starts to manifest in the spectrum, has been excluded from the fitting process and so the $C P E_{\mathrm{G}}$ element has also been removed from the model (The horizontal line with the double arrow heads marks out the frequency range used for the fitting process). This simplified model then provides a good fit to the dielectric relaxation spectrum of ice at frequencies close to, and on the high frequency side of, the dielectric loss peak. Therefore, using this approach, i.e. by 
excluding CPE from the fitting, it might be possible to extract reasonable estimates for the relaxation time $(\tau)$ and distribution parameter $(\alpha)$ from fitting the Cole-Cole function over the middle to high frequency range of the TVIS spectrum
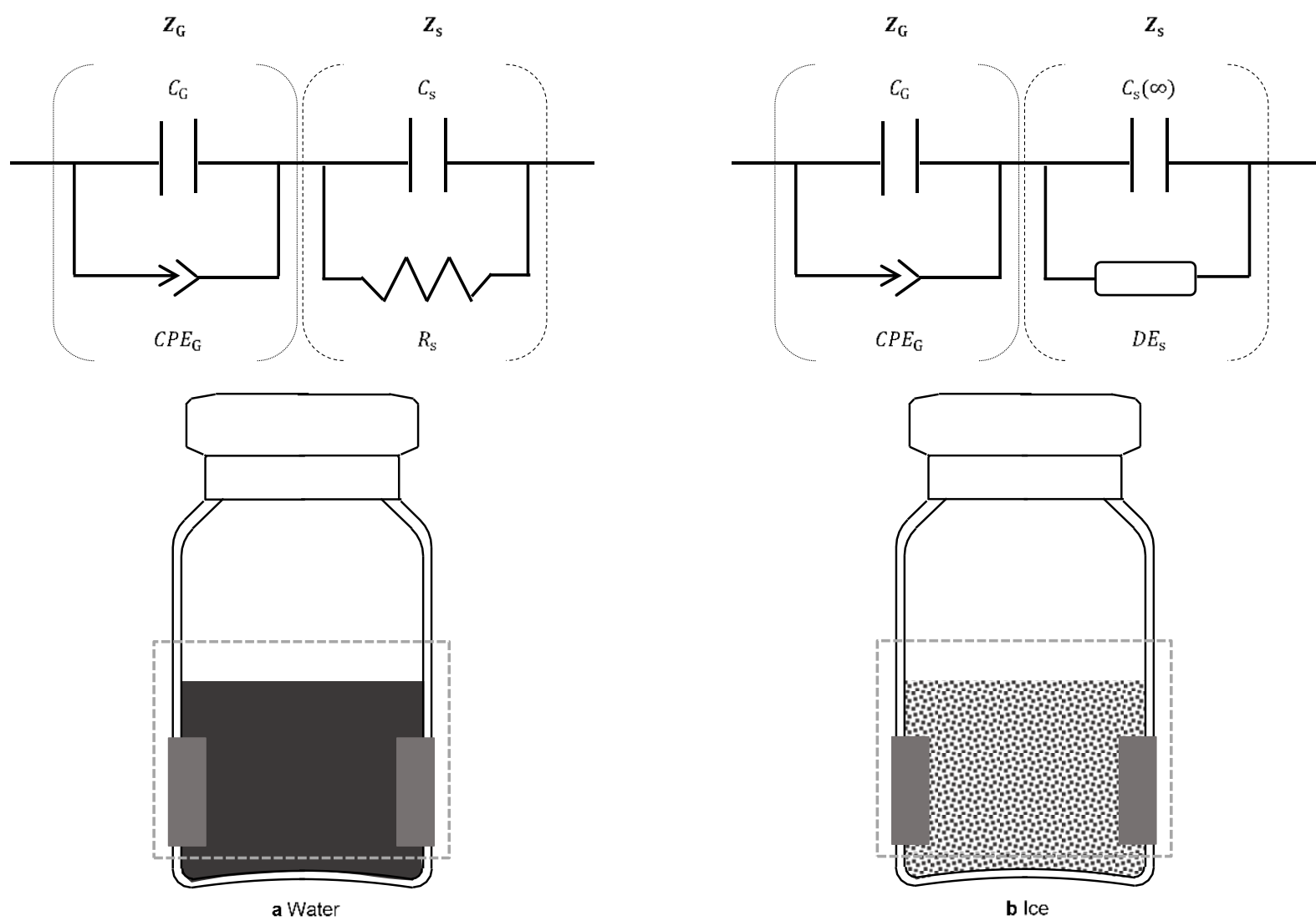

Fig. 16 Model for the TVIS vial containing (a) liquid water where the polarization mechanism association with percolation of protonic charge through the glass wall is modelled by the $C P E_{\mathrm{G}}$ element and the impedance of the water is represented by a resistor $\left(R_{\mathrm{s}}\right)$ and capacitor $\left(C_{\mathrm{s}}\right)$; and $(\mathbf{b})$ frozen water where the impedance of the sample includes the dielectric relaxation of ice (as modelled by the distributed element $D E_{\mathrm{s}}$ of the Cole-Cole type) and an instantaneous capacitance $C_{\mathrm{s}}(\infty)$. The glass wall impedance is assumed to behave like a capacitor $\left(C_{\mathrm{G}}\right)$ with a low frequency dispersive attribute, modeled by the constant phase element, $C P E_{\mathrm{G}}$

Having modelled the impedance spectrum of the ice filled vial, it is then interesting to see what the spectrum would look like if the fit parameters for the ice relaxation and instantaneous capacitance were simulated in the absence of the glass wall capacitance (Fig. 18). It is clear from the differences between the simulated spectrum (grey line) and the experimental data (black dots) that the glass wall capacitance shifts the relaxation peak to higher frequencies and reduces it in size. 

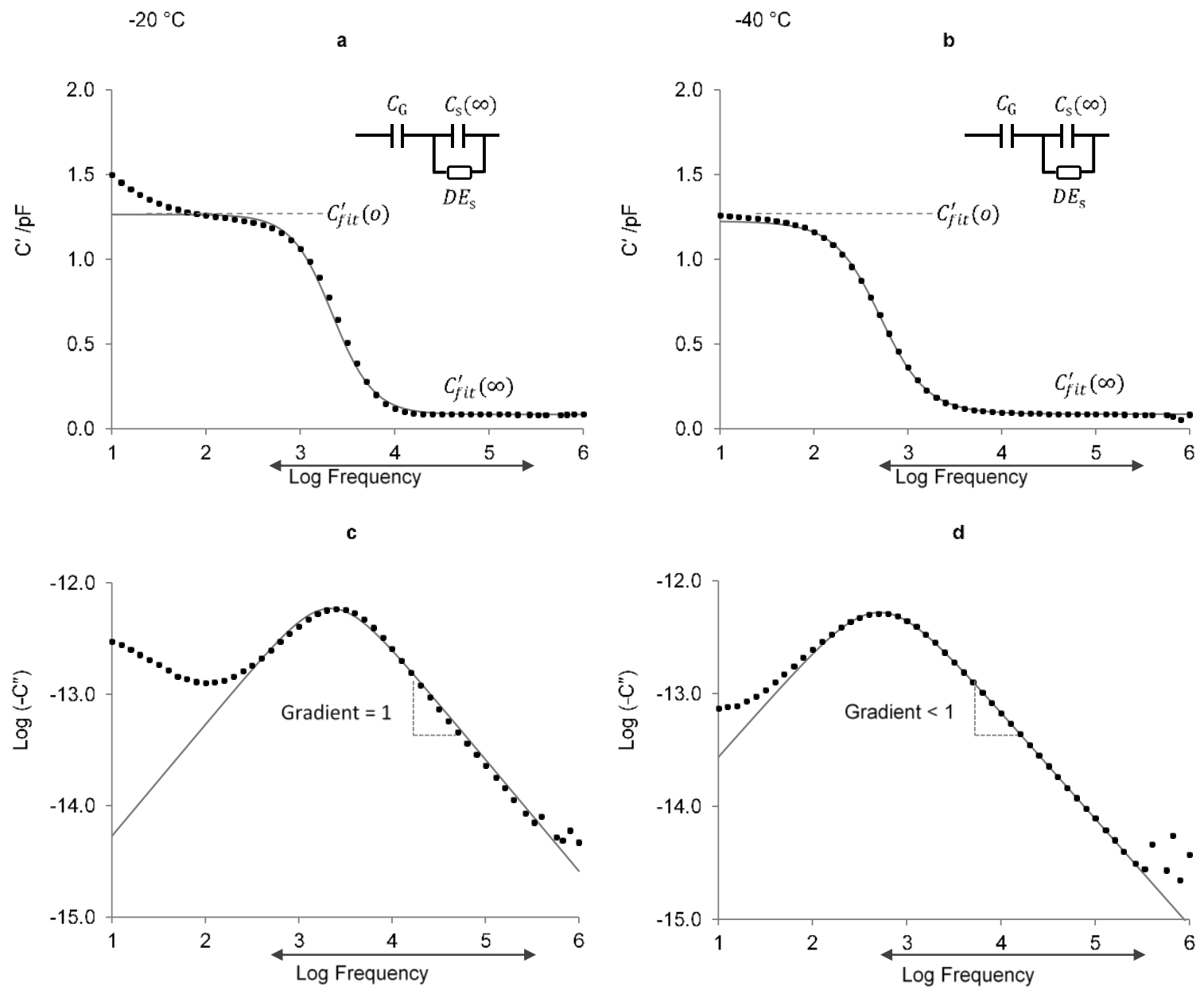

Fig. 17 Measured sample capacitance $C_{\mathrm{s}}(f)$ as a function of frequency (black dots) and fitting results (grey line) to the simplified model for the dielectric relaxation of ice and the glass wall capacitance (which ignores the $C P E_{\mathrm{G}}$ response of the glass) (a to d) for the TVIS vial containing $3.5 \mathrm{~g}$ of double distilled water (fill factor 0.7) with electrodes of dimensions $19 \times 10 \mathrm{~mm}$ and a $3 \mathrm{~mm}$ gap between the bottom of the electrode and base of the vial. The frequency range used for the fitting process is marked by the double-headed arrow. The real part capacitance of ice at (a) $-20{ }^{\circ} \mathrm{C}$ and $(\mathbf{b})-40{ }^{\circ} \mathrm{C}$, and the imaginary part capacitance of ice at $(\mathbf{c})-20^{\circ} \mathrm{C}$ and (d) $-40{ }^{\circ} \mathrm{C}$ are shown. Both temperatures were arrived at on reheating of frozen water from $-45^{\circ} \mathrm{C}$. Note that the imaginary permittivity is plotted on a $\log$ scale in order to demonstrate more clearly the broadening of the relaxation peak as the temperature is reduced from $-20{ }^{\circ} \mathrm{C}$ to $-40{ }^{\circ} \mathrm{C}$. This broadening is manifest in the Cole-Cole distribution parameter $\alpha$ which has a value of $\alpha=1$ at $-20^{\circ} \mathrm{C}$ (which means that the relaxation has a Debye-like characteristics) and a value of $\alpha=0.945$ at $-40{ }^{\circ} \mathrm{C}$, which is consistent with the change in polarization mechanism from orientation charge propagation transferring L-D defects through the ice matrix to ionic charge propagation of $\mathrm{H} 3 \mathrm{O}+$ and $\mathrm{OH}-$ ion pairs 

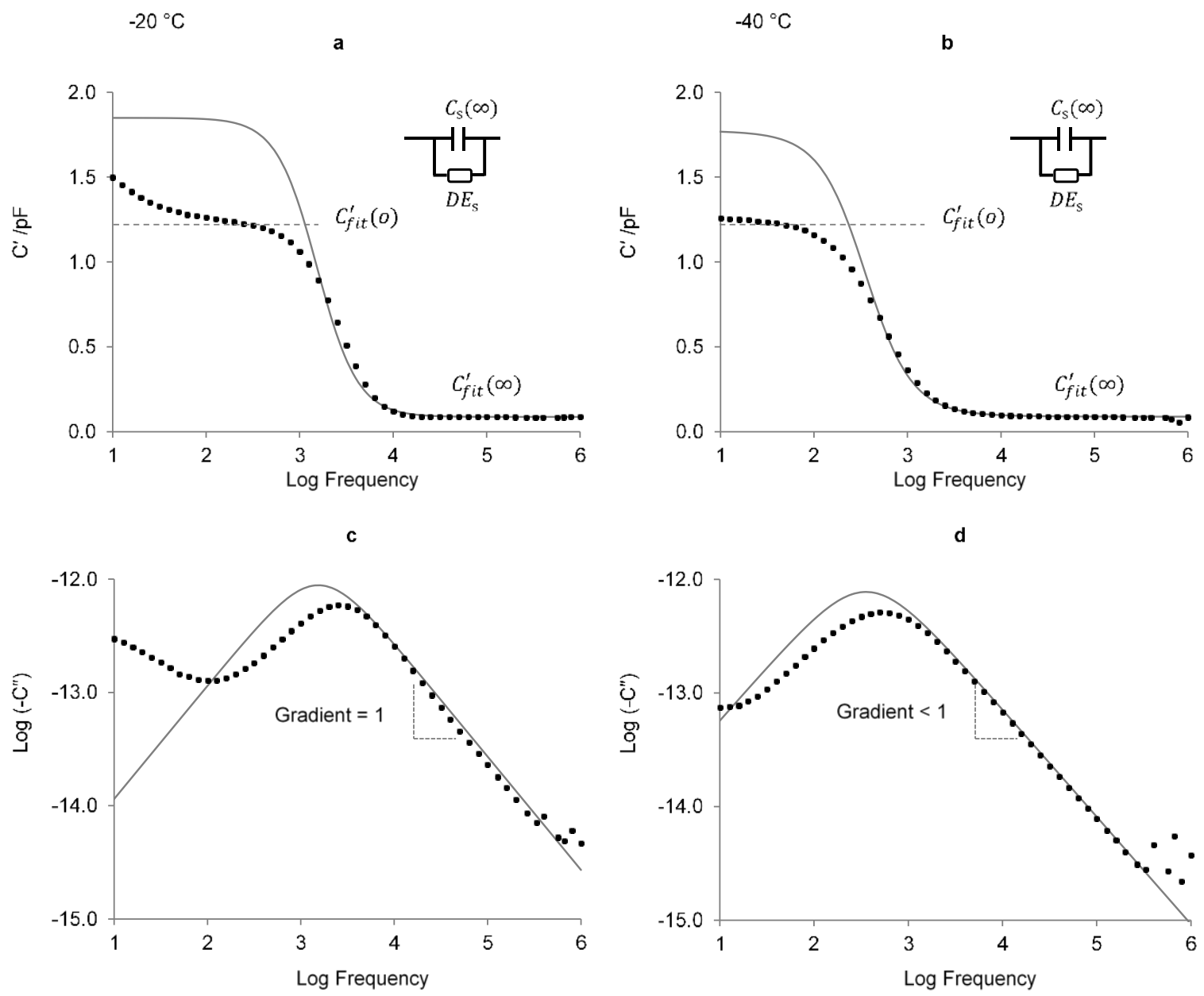

Fig. 18 The capacitance spectrum of $3.5 \mathrm{~g}$ frozen water at $-20{ }^{\circ} \mathrm{C}(\mathbf{a}$ and $\mathbf{c})$ and $-40{ }^{\circ} \mathrm{C}(\mathbf{b}$ and $\mathbf{d})$ in a TVIS vial with electrode dimensions of $10 \times 19 \mathrm{~mm}$ and positioned at $3 \mathrm{~mm}$ from the base of vial are shown. The black dots represent the measured data points whereas the grey line shows the simulation results when the $C_{\mathrm{G}}$ element is removed from the simplified model $\left(C_{\mathrm{G}}-C_{\mathrm{S}}(\infty)=D E_{\mathrm{S}}\right)$ to illustrate the impact of glass capacitance $\left(C_{\mathrm{G}}\right)$ on the peak frequency and peak amplitude of ice at $(\mathbf{c})-20{ }^{\circ} \mathrm{C}$ and (d) $-40{ }^{\circ} \mathrm{C}$

\subsubsection{Determination of the Glass Wall Capacitance $\left(C_{G}\right)$}

In the section on liquids, it was demonstrated how one could determine the cell constant $\left(C_{o}=\right.$ $0.0525 \mathrm{pF})$ from the measurement of a range of liquids and how the interfacial capacitance $\left(C_{G}\right)$ varied slightly between the liquids (in the range 2.98 to $3.07 \mathrm{pF}$ ). These calculations were based on the fact that the low frequency end of the real part capacitance spectrum provided an estimate for the glass wall capacitance $\left(C_{G}\right)$ which could then be inserted into the equation for the high frequency response (Eq. $10)$ to find an estimate for the sample capacitance $\left(C_{s}\right)$. Plotting sample capacitance as a function of the dielectric permittivity of a range of polar liquids then provided the estimate for the cell constant.

For ice, the response is somewhat different, given that the dispersion is due to a dielectric relaxation rather than the Maxwell-Wagner polarization of the glass wall capacitance. Here, in the limit of low frequency $(f \rightarrow 0)$ the capacitance of the model for ice (Fig. 17) can be written as:

$$
C_{f i t}^{\prime}(0)=\frac{C_{S}(0) \cdot C_{G}}{C_{S}(0)+C_{G}}
$$


where $C_{s}(0)$ has a value equal to the sum of the magnitude of $D E_{\mathrm{s}}$ and $C_{\mathrm{s}}(\infty)$. Incidentally, in the limit of high frequency $(f \rightarrow \infty)$ the capacitance of the model in Fig. 17 can be written as:

$$
C_{f i t}^{\prime}(\infty)=\frac{C_{S}(\infty) \cdot C_{G}}{C_{S}(\infty)+C_{G}}
$$

An expression for the glass wall capacitance $C_{\mathrm{G}}$ (Eq. 22) can be found by re-arranging Eq. 17 as follows:

$$
\begin{gathered}
C_{f i t}^{\prime}(0)=\frac{C_{\mathrm{s}}(0) \cdot C_{\mathrm{G}}}{C_{\mathrm{s}}(0)+C_{\mathrm{G}}} \cdot \frac{C_{\mathrm{s}}(0)-C_{\mathrm{G}}}{C_{\mathrm{s}}(0)-C_{\mathrm{G}}} \\
C_{f i t}^{\prime}(0)=\frac{C_{s}^{2}(0) C_{G}-C_{s}(0) C_{G}^{2}}{C_{\mathrm{s}}^{2}(0)-C_{\mathrm{G}}^{2}} \\
\left(C_{\mathrm{s}}(0)-C_{f i t}^{\prime}(0)\right) C_{\mathrm{G}}^{2}-C_{\mathrm{s}}^{2}(0) C_{\mathrm{G}}+C_{f i t}^{\prime}(0) C_{\mathrm{s}}^{2}(0)=0 \\
C_{G}=\frac{-\left(C_{s}^{2}(0)\right) \pm \sqrt{\left(C_{\mathrm{s}}^{2}(0)\right)^{2}-4\left(C_{f i t}^{\prime}(0)-C_{\mathrm{s}}(0)\right)\left(C_{f i t}^{\prime}(0) C_{\mathrm{s}}^{2}(0)\right)}}{2\left(C_{f i t}^{\prime}(0)-C_{s}(0)\right)}
\end{gathered}
$$

One can then estimate the dielectric properties of the ice-glass wall interface $\left(C_{G}\right)$ by first fitting the complex capacitance spectrum to find estimates for the low frequency capacitance $\left(C_{f i t}^{\prime}(0)\right)$ at various temperatures and then inserting those estimates into Eq. 22 along with estimates for the low frequency capacitance of the ice, which have been calculated from literature values of the dielectric properties of ice $\left(\varepsilon_{S}(0)\right)$ (Popov et al. 2017) as shown in Table 4 and the cell constant which has been determined in the section on liquids.

Estimate for the glass capacitance $C_{\mathrm{G}}$ at different temperatures during the re-heating of ice is shown in Table 4 and Fig. 19.

Table 4 Estimate for the instantaneous capacitance of the glass wall $\left(C_{\mathrm{G}}\right)$ during the re-heating of ice (for ultrapure water $6.5 \mathrm{~g}$ is filled in an Adelphi VC010-20C Type I glass tubing vial attached with a pair of electrodes of dimensions $19 \times 10 \mathrm{~mm}$ and $3 \mathrm{~mm}$ gap between the bottom of the electrode and base of the vial).(Popov et al. 2017)

\begin{tabular}{c|ccccc}
\hline Temperature $/{ }^{\circ} \mathrm{C}$ & $C^{\prime}(0) / \mathrm{pF}$ & $\varepsilon_{S}(0)$ & $C_{S}(0) / \mathrm{pF}$ & $C_{G} 1 / \mathrm{pF}$ & $C_{G} 2 / \mathrm{pF}$ \\
\hline-51.8 & 1.65 & 117.6 & 6.17 & 2.25 & 6.17 \\
\hline-50.1 & 1.65 & 116.7 & 6.12 & 2.26 & 6.12 \\
\hline-45.1 & 1.65 & 114.0 & 5.99 & 2.28 & 5.99 \\
\hline-39.9 & 1.65 & 111.4 & 5.85 & 2.31 & 5.85 \\
\hline-35.1 & 1.66 & 109.1 & 5.73 & 2.33 & 5.73 \\
\hline-29.9 & 1.66 & 106.7 & 5.60 & 2.37 & 5.60 \\
\hline-24.9 & 1.67 & 104.5 & 5.49 & 2.40 & 5.49 \\
\hline-20.1 & 1.67 & 102.5 & 5.38 & 2.42 & 5.38 \\
\hline
\end{tabular}


The value of $C_{\mathrm{G}}$ taken from Eq. 22 has two values $\left(C_{\mathrm{G}} 1\right.$ and $\left.C_{\mathrm{G}} 2\right)$. However, $C_{\mathrm{G}} 1$ is selected as a parameter for $C_{\mathrm{G}}-C_{\mathrm{S}}=D E_{\mathrm{S}}$ model because it has the correct temperature dependency (i.e. $C_{\mathrm{G}}$ increases with temperature) as shown in Fig. 19 and its values are also lower than both the theoretical $\left(C_{\mathrm{G}}=3.99\right.$ $\mathrm{pF})$ and experimental values $\left(C_{\mathrm{G}}=2.98-3.07 \mathrm{pF}\right)$. Moreover, a linear extrapolation of these low temperature values to $+20^{\circ} \mathrm{C}$ (i.e. the temperature at which the liquid water measurements were made) predicts a value for $C_{\mathrm{G}}$ of $\sim 2.65 \mathrm{pF}$ which is close to that obtained experimentally for liquid water $\left(C_{\mathrm{G}}\right.$ $=3.07 \mathrm{pF})$. A second order polynomial fit to the low temperature values of $C_{\mathrm{G}}$ provides an estimate $\left(C_{\mathrm{G}}\right.$ $=2.76 \mathrm{pF}$ ) which is even closer to that obtained for liquid water.
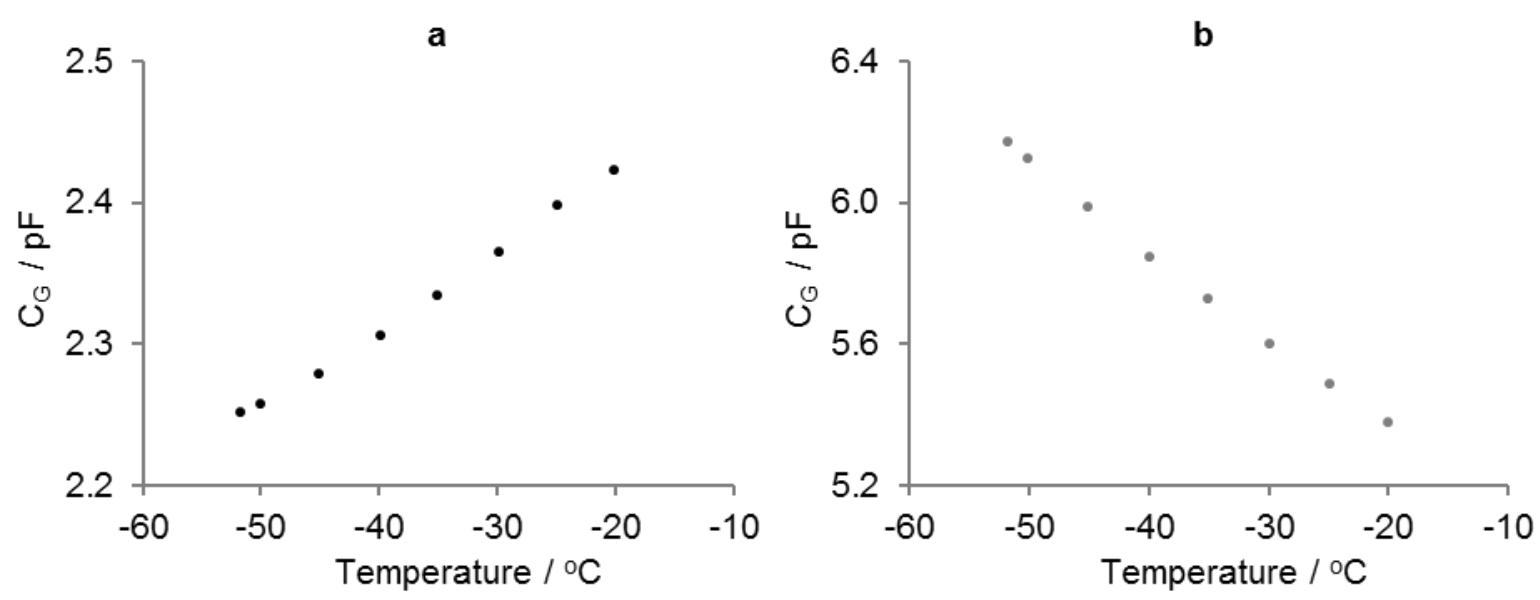

Fig. 19 Estimates for the instantaneous capacitance of the glass wall over the temperature range -20 to $-50{ }^{\circ} \mathrm{C}$; $\mathbf{a}$ is $C_{\mathrm{G}} 1$ and $\mathbf{b}$ is $C_{\mathrm{G}} 2$

\subsubsection{Freezing of Solutions}

As ice forms during the freezing stage, the remaining water becomes concentrated into an unfrozen fraction which contains the solutes from the original solution. The solutes are either in an amorphous super-cooled liquid/glass (depending on whether the temperature of this fraction is below its glass transition temperature) or will have precipitated from solution (crystallised) as they exceed their solubility limit. Given the high viscosity of this phase and the tortuosity of the path length for the conduction of ionic charges then its conductivity is expected to be dramatically lower than that of the original liquid phase (even allowing for the fact that the concentration of ionic conductors in this phase is higher that of the original solution). However, the conductivity of the unfrozen fraction is likely to be higher than ice and so there is potential for the re-introduction of a Maxwell-Wagner process to the complex capacitance spectrum of a frozen solution. Figure 20 shows an example of such a response.

Two possible mechanisms provide feasible explanations of the origin of this second process. The first is that the juxtaposition of ice crystals with the thin interstitial spaces of the unfrozen results in Maxwell-Wagner polarization at the mesoscale. The second is that the conductivity pathways for charges migrating through the unfrozen fraction to the extremities of the sample result in a Maxwell-Wagner process that is manifest at the macroscopic scale of the glass-sample interface. The reason why it is manifest in the lower frequency part of the spectrum is a consequence of the much higher resistance of the unfrozen fraction compared with that of the original solution.

The equivalent circuit must then be modified to account for this second process by reintroducing a resistance, $\mathrm{R}$, in parallel with the Cole-Cole element for the relaxation of ice (Fig. 21) 
a

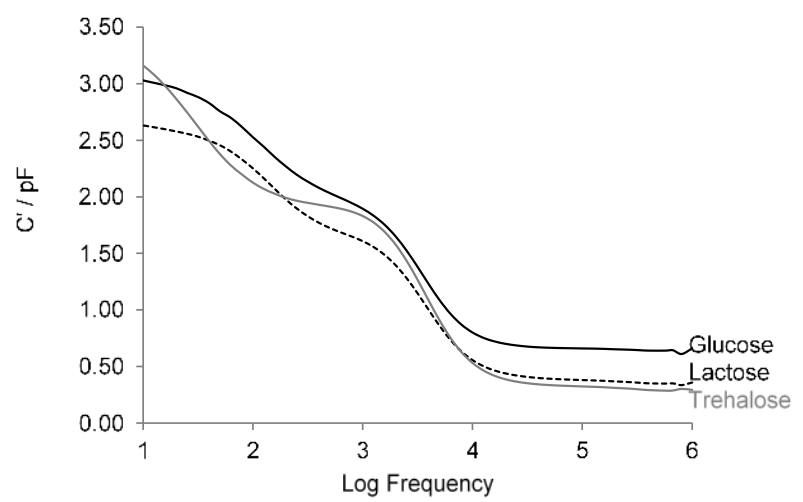

b

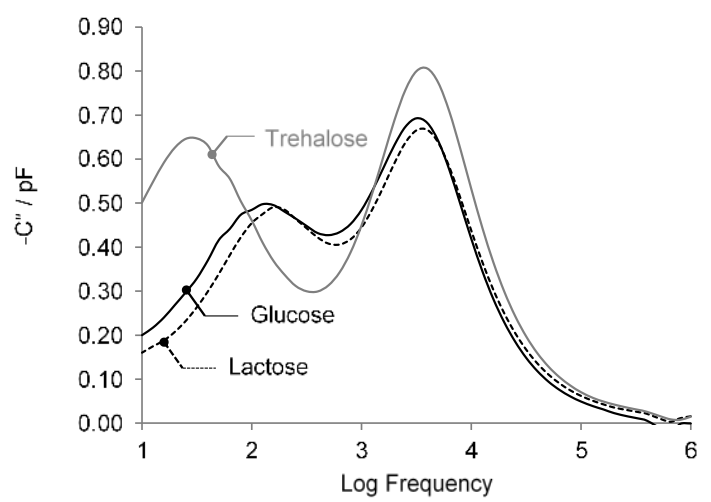

Fig. 20 The capacitance spectra of 3\% solutions of glucose (black solid line), lactose (black dash line) and trehalose (grey solid line) contained in the TVIS vial with electrode dimension of $10 \times 19 \mathrm{~mm}$ and at a height of $3 \mathrm{~mm}$ from vial base during re-heating process. The temperature of the samples is -18 ${ }^{\circ} \mathbf{C}$. The real part and imaginary part capacitance are shown in $\mathbf{a}$ and $\mathbf{b}$, respectively
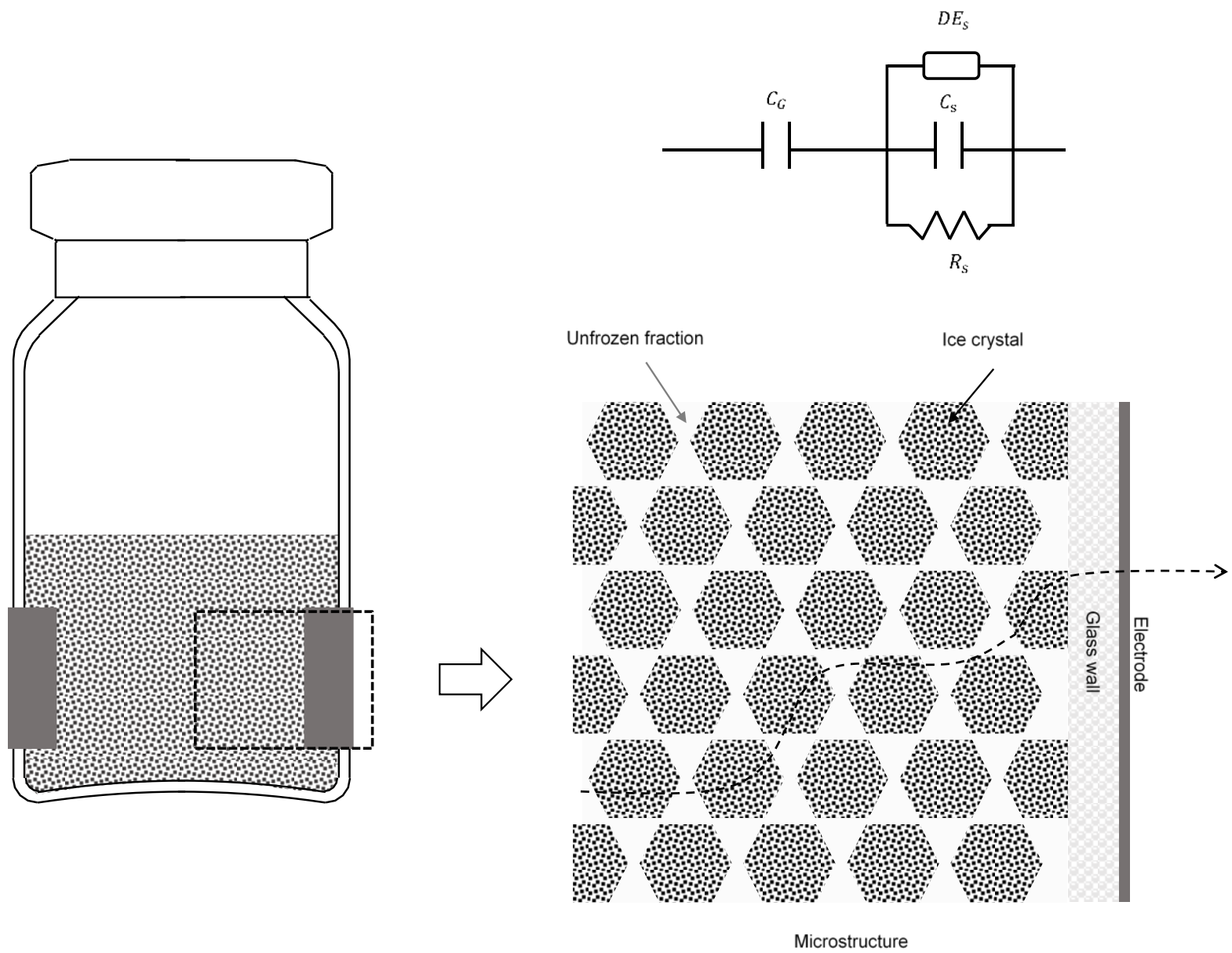

Fig. 21 A more complex equivalent circuit model is required for the two phase system comprising ice fraction and unfrozen fraction

Complete solidification of all components (not just that of the ice) is achieved by cooling the contents of the vial to temperatures lower than the eutectic temperature of the crystalline solutes and/or the glass transition temperatures of the non-crystallizing solutes. Combinations of non- 
crystallizing excipients and crystallizing excipients has been used to suppress the crystallization of the latter and it has been demonstrated that TVIS may be used to witness the crystallization of a eutectic material while being able to monitor the suppression of the eutectic by the addition of another agent such as sucrose (Arshad et al. 2014).

Previous publications on various applications of TVIS technology for frozen solutions, describe the uses of TVIS for determining (1) the glass transition temperature of maltodextrin solutions (Smith et al. 2013b); (2) the potential application of TVIS in mini pilot studies (Smith et al. 2017); (3) the impact of annealing on primary drying rate (Smith, Arshad, Polygalov and Ermolina 2014b).

\section{Future Prospects}

The TVIS technology described in this chapter is currently being developed further for the purpose of establishing new approaches for determining a number of critical process parameters:

1 The distribution of temperature, in the vertical direction within the vial, such that the sublimation interface temperature and the base temperature inside the vial might be predicted

2 The sublimation rate in primary drying

3 The heat transfer coefficient $\left(K_{v}\right)$ and the dry layer resistance $\left(R_{p}\right)$

4 The impact of microcollapse.

A commercial system has now been developed by Sciospec GmbH which replicates the capability of De Montfort University's laboratory demonstrator that has been used to create the data throughout this book chapter, but with the additional benefit of an extended frequency range to $10 \mathrm{MHz}$ and a temperature stabilized operational amplifier to reduce instrumentation drift.

Further developments in the instrumentation, with ever increasing sensitivity to small capacitances will enable the electrode system, to be removed from the vial and a new single electrode to be placed over a small cluster of vials in a non-contact or stand-off mode. A "noncontact" through vial impedance spectrometer of this type (NC-TVIS) would be compatible with the automatic vial loading system. Its limitation is that it may not be compatible with the vial stoppering system unless the electrode system is integrated within the dryer, e.g. built into the underside of the shelf above the vials. In order to provide compatibility with both automatic vial loading and vial stoppering then a further development of the TVIS system is envisaged that can be pushed into the dryer along with the other vials, in a similar way to the operation of the Lyo Shuttle that was developed by Ellab for thermocouple measurements. The operation principles of a 'TVIS Shuttle' would be similar to the TVIS system described in this book chapter and would rely on modified vials with electrodes attached to the outside surface. The instrument (which is now located inside the dryer) would need to be stable within the environment of low temperature and pressure, while generating minimal heat so as to have an insignificant impact on the heat transfer to the nearest neighbour vials, including the TVIS measurement vial. Data from the device could be downloaded after completion of the process, and the instrument removed from the dryer, or transmitted by RF or some other protocol during the freeze-drying process. 


\section{Further Reading}

While applications for impedance spectroscopy in the pharmaceutical sciences are quite limited in scope and application, there are more generalized applications for impedance spectroscopy and its sister technique of broadband dielectric spectroscopy, in a wide range of applications, including electrochemistry, materials science and biology

1. Raicu V, Feldman Y (eds) (2015) Dielectric relaxation in biological systems: physical principles, methods, and applications. Oxford University Press, Oxford.

2. Orazem ME, Tribollet B (2008) Electrochemical impedance spectroscopy. John Wiley \& Sons, New Jersey.

3. Barsoukov E, Macdonald JR (eds) (2005) Impedance spectroscopy: theory, experiment, and applications, 2 edn. Wiley-Interscience, a John Wiley \& Sons, Inc., New Jersey.

4. Kremer F, Schönhals A (eds) (2003) Broadband dielectric spectroscopy. Springer, Berlin, Heidelberg.

\section{Acknowledgement}

The original TVIS system used to generate the spectra within this book chapter was developed by Evgeny Polygalov and Geoff Smith (from De Montfort University) in a collaboration with GEA Pharma Systems (Eastleigh, UK) and was part-funded by a UK government, Innovate UK Collaborative R\&D project called LyoDEA (Project Reference: 100527).

Special thanks go to Yowwares Jeeraruangrattana (from the Government Pharmaceutical Organization, in Thailand and one of De Montfort University's PhD students from 2015 to 2018) for creating the majority of the images, drawings and figures, and assisting in formatting of the text and bibliography.

\section{References}

Alkeev N, Averin S, von Gratowski S (2015) New Method for Monitoring the Process of Freeze Drying of Biological Materials. AAPS Pharmscitech 16(6):1474-1479.

Arshad MS, Smith G, Polygalov E et al (2014) Through-vial impedance spectroscopy of critical events during the freezing stage of the lyophilization cycle: the example of the impact of sucrose on the crystallization of mannitol. Eur J Pharm Biopharm 87(3):598-605. doi:10.1016/j.ejpb.2014.05.005.

Bandari S, Sesha Sai M, Reddy YR (2013) Optimization of lyophilization cycles for gemcitabine. Int J Pharm Pharm Sci 5(2):216-221.

Bernal J, Fowler R (1933) A theory of water and ionic solution, with particular reference to hydrogen and hydroxyl ions. J Chem Phys 1(8):515-548.

Carlson G, Illman B (2002) Series capacitors and the inverse sum rule. Am J Phys 70(11):1122-1128.

DeLuca PP (1977) Freeze drying of pharmaceuticals. J Vac Sci Technol 14(1):620-629.

Deluca P, Lachman L (1965) Lyophilization of pharmaceuticals I. Effect of certain physical-chemical properties. J Pharm Sci 54(4):617-624. 
Evans SA, Morris KR, Mackenzie AP et al (1995) Dielectric characterization of thermodynamic first order events in model frozen systems intended for lyophilization. PDA J Pharm Sci Technol 49(1):2-8.

Foster K.R. SHP (1989) Dielectric properties of tissues and biological materials: A critical review. Crit Rev Biomed Eng 17:25-104.

Greaves RIN (1954) Theoretical aspects of drying by vacuum sublimation. In: Harris RJC (ed) Biological applications of freezing and drying. Academic Press, New York, p 87-127.

Her LM, Jefferis RP, Gatlin LA et al (1994) Measurement of glass transition temperatures in freeze concentrated solutions of non-electrolytes by electrical thermal analysis. Pharm Res 11(7):1023-1029.

Hobbs PV (1974) Ice physics. Claredon press, Oxford.

Jacob JM (2004) Sinusoidal waveforms. In: Anonymous Advanced AC circuits and electronics: principles \& applications Delmar Learning, New York, p 3-28.

Johari GP, Whalley E (1981) The dielectric properties of ice Ih in the range 272-133 K. J Chem Phys 75(3):1333-1340.

Liu J, Duan CG, Mei WN et al (2005) Dielectric properties and maxwell-wagner relaxation of compounds $A \mathrm{Cu}_{3} \mathrm{Ti}_{4} \mathrm{O}_{12}\left(A=\mathrm{Ca}, \mathrm{Bi}_{23}, \mathrm{Y}_{23}, \mathrm{La}_{2 \beta}\right)$. J Appl Phys 98(9):093703.

Mackenzie AP (1985) Changes in electrical resistance during freezing and their application to the control of the freeze-drying process. In: Anonymous Fundamentals and applications of freeze-drying to biological materials, drugs and foodstuffs. International Institute of Refrigeration, Paris, p 155-163.

Melinder $\AA$ (2010) Properties and other aspects of aqueous solutions used for single phase and ice slurry applications. Int J Refrig 33(8):1506-1512.

Morris KR, Evans SA, Mackenzie AP et al (1994) Prediction of lyophile collapse temperature by dielectric analysis. PDA J Pharm Sci Technol 48(6):318-329.

Pauling L, Brockway LO, Beach JY (1935) The dependence of interatomic distance on single bonddouble bond resonance. J Am Chem Soc 57(12):2705-2709.

Popov I, Lunev I, Khamzin A et al (2017) The low-temperature dynamic crossover in the dielectric relaxation of ice I h. Phys Chem Chem Phys 19(42):28610-28620.

Popov I, Puzenko A, Khamzin A et al (2015) The dynamic crossover in dielectric relaxation behavior of ice I h. Phys Chem Chem Phys 17(2):1489-1497.

Rey LR (1960a) Study of the freezing and drying of tissues at very low temperatures. In: Parkes AS, Audrey US (eds) Recent research in freezing and drying Blackwell, Oxford, p 40-62.

Rey LR (1960b) Thermal analysis of eutectics in freezing solutions. Ann N Y Acad Sci 85(1):510-534.

Ryabov Y, Gutina A, Arkhipov V et al (2001) Dielectric relaxation of water absorbed in porous glass. J Phys Chem B 105(9):1845-1850.

Shen M, Ge S, Cao W (2001) Dielectric enhancement and maxwell-wagner effects in polycrystalline ferroelectric multilayered thin films. J Phys D: Appl Phys 34(19):2935-2938. 
Shinyashiki N, Yamamoto W, Yokoyama A et al (2009) Glass transitions in aqueous solutions of protein (bovine serum albumin). J Phys Chem B 113(43):14448-14456.

Smith G, Arshad MS, Nazari K et al (2014) Through-vial impedance spectroscopy: a new in-line process analytical technology for freeze drying. Pharm Technol 38(4).

Smith G, Arshad MS, Polygalov E et al (2014a) Factors affecting the use of impedance spectroscopy in the characterisation of the freezing stage of the lyophilisation process: the impact of liquid fill height in relation to electrode geometry. AAPS PharmSciTech 15(2):261-269.

Smith G, Arshad MS, Polygalov E et al (2014b) Through-vial impedance spectroscopy of the mechanisms of annealing in the freeze-drying of maltodextrin: the impact of annealing hold time and temperature on the primary drying rate. J Pharm Sci 103(6):1799-1810.

Smith G, Arshad MS, Polygalov E et al (2017) Process understanding in freeze-drying cycle development: applications for through-vial impedance spectroscopy (tvis) in mini-pilot studies. J Pharm Innov 12(1):26-40.

Smith G, Polygalov E, Arshad M et al (2013a) An impedance-based process analytical technology for monitoring the lyophilisation process. Int J Pharm 449(1):72-83.

Smith G, Arshad MS, Polygalov E et al (2013b) An application for impedance spectroscopy in the characterisation of the glass transition during the lyophilization cycle: the example of a $10 \% \mathrm{w} / \mathrm{v}$ maltodextrin solution. Eur J Pharm Biopharm 85(3, Part B):1130-1140. doi:10.1016/j.ejpb.2013.08.004.

Stubbe M, Gimsa J (2015) Maxwell's mixing equation revisited: characteristic impedance equations for ellipsoidal cells. Biophys J 109(2):194-208. doi:10.1016/j.bpj.2015.06.021.

Ward KR, Matejtschuk P (2010) The use of microscopy, thermal analysis, and impedance measurements to establish critical formulation parameters for freeze-drying cycle development. In: Rey L, May JC (eds) Freeze drying/lyophilization of pharmaceutical and biological products, 3 edn. Informa Healthcare, London, p 111-135. 


\section{Annex 1 Analysis of Peak Frequency and Peak Magnitude}

The relative simplicity of the imaginary capacitance spectrum means that it's is possible to follow two features of the peak using proprietary LyoView ${ }^{\mathrm{TM}}$ data viewing software, namely the peak frequency $\left(F_{P E A K}\right)$ and the peak amplitude $\left(C_{P E A K}^{\prime \prime}\right)$.

\section{LyoView ${ }^{\mathrm{TM}}$ Peak Finding Procedure}

This procedure is intended for the determination of the Maxwell-Wagner peak parameters such as peak amplitude and frequency position for individual dielectric spectra obtained during the freeze-drying cycle.

There are four stages in peak search procedure:

1. Preparation of the spectrum: (1) Smoothing of the dielectric loss spectrum to eliminate sharp spikes originating from scattering due to finite sensitivity or from electromagnetic interference. For this purpose the RMS deviation throughout the curve is calculated. For each point deviation from average of previous and next point is calculated and if the deviation is larger than half of RMS the value point is replaced by the average of previous and next point. After that moving average filter with aperture 3 is applied twice and using free cubic spline the number of points $\mathrm{N}$ is increased to $10^{*} \mathrm{~N}$ ); followed by (2) interpolation by free cubic spline in order to increase the number of points and accuracy of peak parameters determination.

2. Differentiation of the spectrum obtained in step 1 and finding all negative peaks (zero $1^{\text {st }}$ derivative and positive $2^{\text {nd }}$ derivative) and all positive peaks ( $2^{\text {nd }}$ derivative negative) peaks which are present in the smoothed spectrum.

3. Rejection of any narrow peaks that originate from residual spikes which are not completely suppressed by curve smoothing.

4. Selection of the principle peak, if there is more than one peak that meets all previous criteria. Several criteria are applied depending on the stage of the freeze-drying: (1) at freezing and early stages of primary drying the peak with the largest amplitude is selected, (2) at the middle and late stage of primary drying, the peak with frequency position closest to frequency position of principal peak in previous spectrum is selected.

The $F_{P E A K}$ parameter can be used to predict the product temperature whereas the $C_{P E A K}^{\prime \prime}$ parameter can be used to determine the amount of ice remaining during the primary drying phase from which one can then predict both drying rate and end point. Both parameters lend themselves to the determination of phase behavior (ice formation, eutectic formation and glass transition events). 


\section{Annex 2 Basic Principles of Impedance Spectroscopy as It Applies to a Liquid Filled in TVIS Vial}

The impedance of most real objects (i.e. those which have a combination of characteristics, for example, some resistance and some electrical capacitance) takes on a frequency-dependence largely because the impedance of a capacitance (Fig. 22a) is dependent on the frequency of the applied field, whereas an ideal resistor has zero frequency dependence (Fig. 22b). The impedance spectrum of a capacitor (in terms of the impedance magnitude and phase angle) displays a characteristic negative slow of -1 in the plot of log impedance magnitude vs $\log$ frequency. This is because the impedance is an inverse function of the capacitance and the applied frequency.

$$
Z=\frac{1}{i \omega C}
$$
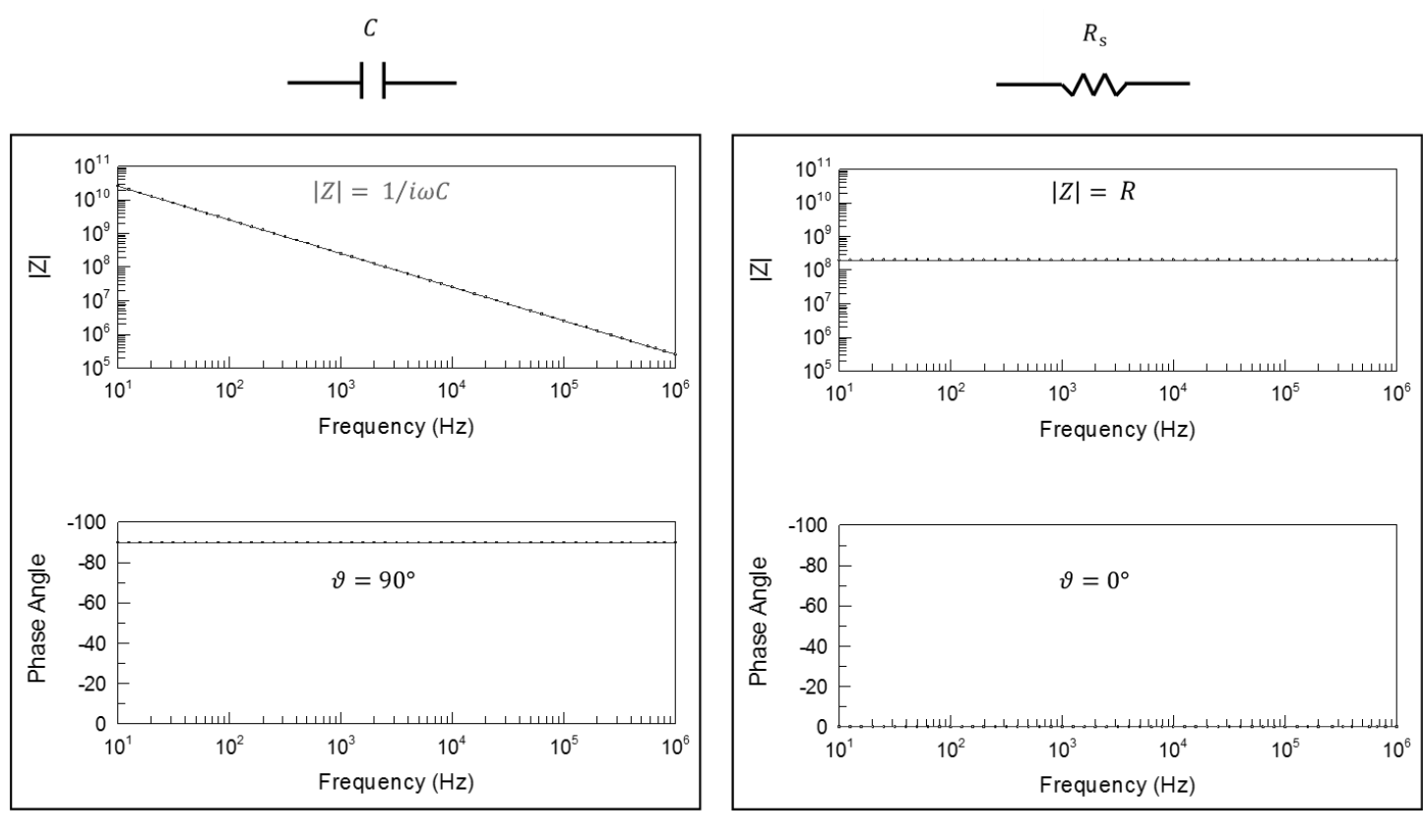

Fig. 22 Bode plots (impedance magnitude $(|Z|)$ and phase angle $(\vartheta)$ ) for the impedances of a capacitor (a) and a resistor $(\mathbf{b})$

In the case of a composite object which has capacitance and resistance then the impedance spectrum that results will be dominated by one or the other element, depending on the whether the elements are in series or in parallel and how the frequency of the applied field defines the relative magnitude of the impedance of the capacitance and the resistance.

\section{In a Series Circuit}

At low frequency the capacitor dominates the spectrum (Fig. 23a) because the impedance of the capacitance is so high that the capacitor effectively controls the current that flows through the circuit.

At high frequency the resistor dominates the spectrum (Fig. 23a) because the impedance of the capacitor has fallen below that of the resistor such that the resistor effectively controls the current that flows through the circuit. 


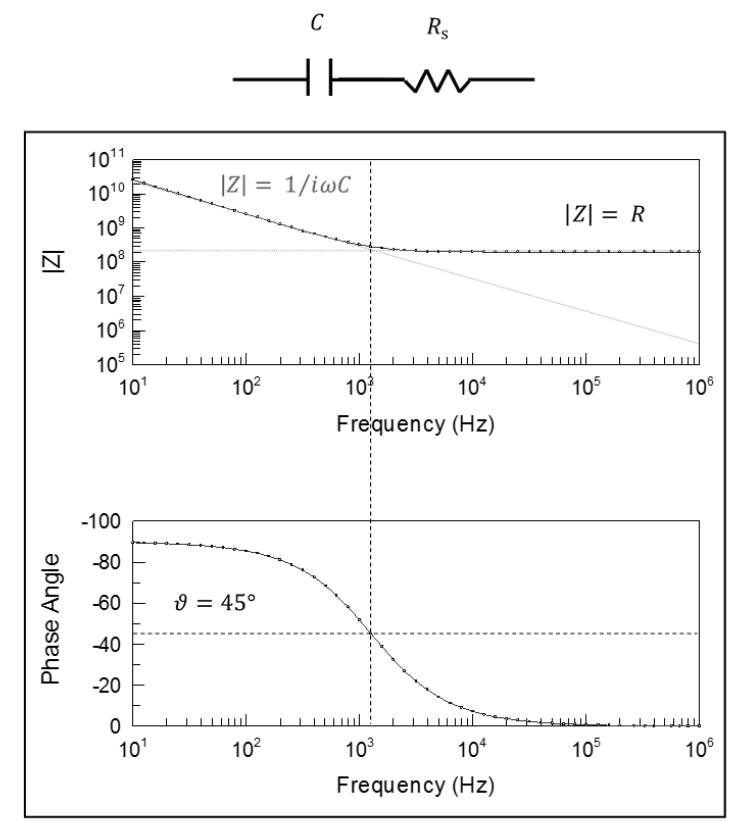

a

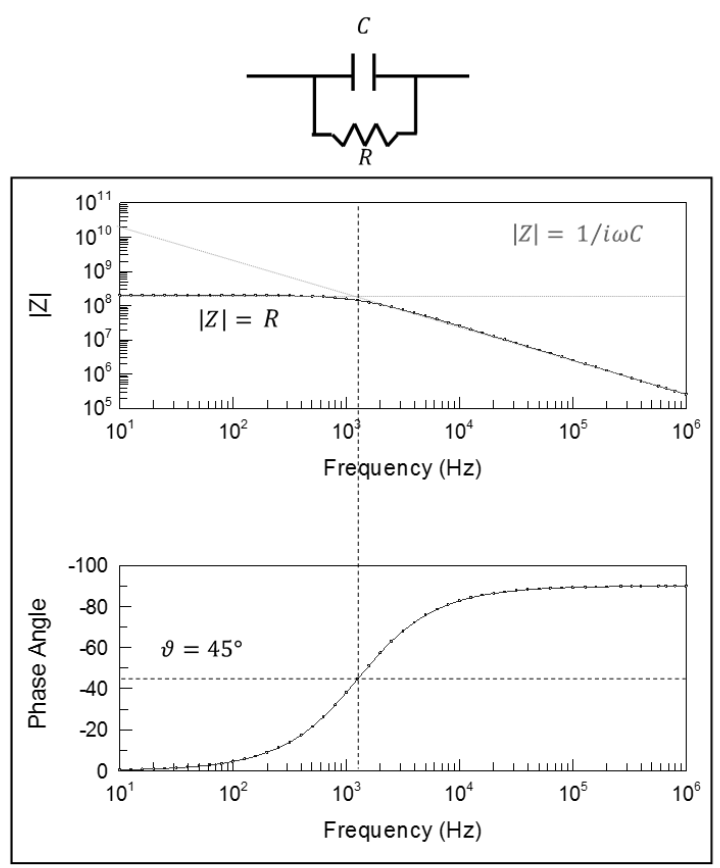

b

Fig. 23 Bode plots (impedance magnitude $(|Z|)$ and phase angle $(\vartheta))$ for the impedances of a series circuit (a) and a parallel circuit (b)

\section{In a Parallel Circuit}

At low frequency the resistor dominates the spectrum (Fig. 23b) because the impedance of the capacitance is so high that all the current flows through the resistor.

At high frequency the capacitor dominates the spectrum (Fig. 23b) because the impedance of the capacitance is now lower than the resistor such that all the current now flows through the capacitor.

More complex composite objects can be considered as combinations of impedances. Again, the impedance spectrum that results will be dominated by one or the other impedance.

\section{In a Complex Circuit}

At low frequency ( $<1 \mathrm{kHz}$ ) the resistor $\mathrm{R}$ (Fig. 24) dominates the impedance of the $R C_{1}$ circuit, because this circuit is in series with a capacitor, $C_{2}$ (which has a high impedance at low frequency) then $C_{2}$ effectively controls the current that flows through the entire circuit

At intermediate frequency (1-30 kHz) the impedance of $C_{2}$ drops below that of the resistor (Fig. 24 ), such that the resistor begins to dominate the impedance and therefore the phase angle tends to increase from -90 to zero (Fig. 24). 


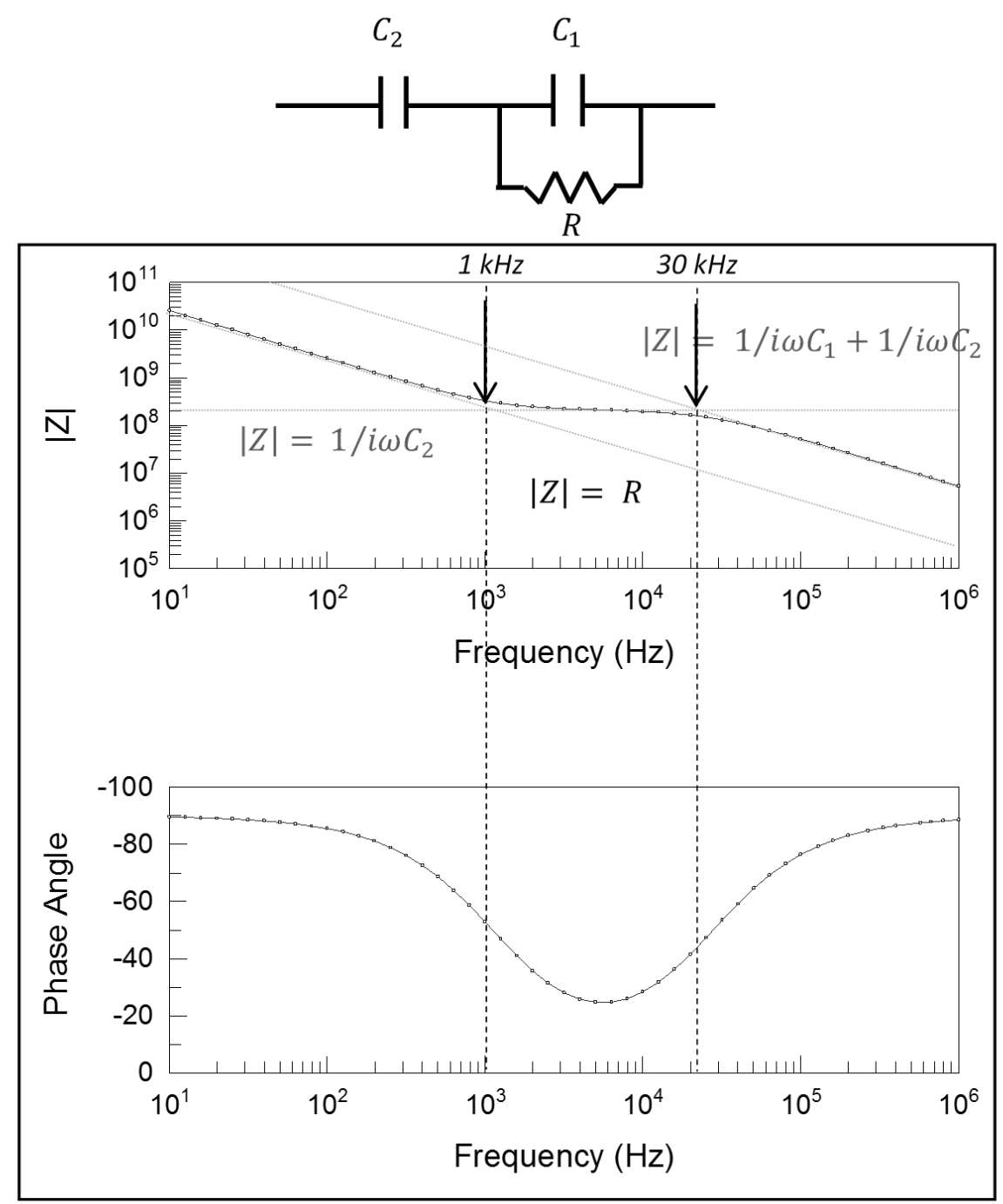

Fig. 24 Bode plots (impedance magnitude $(|Z|)$ and phase angle $(\vartheta))$ for the impedances of a complex circuit

At high frequency $(>30 \mathrm{kHz})$ the impedance of the capacitor $\left(C_{1}\right)$ in parallel with the resistor, decreases below that of the resistor (Fig. 24) such that the resistor no longer dominates the impedance of the parallel $R C_{1}$ circuit and as a result the circuit behaves like two capacitors in series. The high frequency impedance is therefore dominated by the inverse of the sum of the capacitances

$$
Z=\frac{1}{i \omega\left(C_{1}+C_{2}\right)}
$$

The above model can be mapped onto the physical characteristic of the liquid filled vial, where $C_{2}$ is the glass wall capacitance $\left(C_{\mathrm{G}}\right), C_{1}$ is the sample capacitance $\left(C_{\mathrm{S}}\right)$ and $R$ is the sample resistance $\left(R_{\mathrm{s}}\right)$.

The complex impedance $\left(\mathrm{Z}^{*}\right)$ of the model can be calculated from the Eq. 25: 


$$
Z^{*}=\frac{1}{i \omega C^{*}}=\frac{1}{i \omega C_{G}}+\frac{1}{\frac{1}{R_{S}}+i \omega C_{S}}=\frac{1+i \omega R_{S}\left(C_{G}+C_{S}\right)}{i \omega C_{G}-\omega^{2} R C_{G} C_{S}}
$$

where $\omega$ is the frequency of the applied field in radians per second (this angular frequency can be converted into frequency in cycles per second through the relationship $\omega=2 \pi f$ ). The impedance of this model can also be expressed in terms of a complex capacitance $\left(C^{*}\right)$ according to Eq. 26:

$$
C^{*}=C^{\prime}+i C^{\prime \prime}=\frac{1}{i \omega Z^{*}}=\frac{C_{G}+i \omega R_{S} C_{G} C_{S}}{1+i \omega R_{S}\left(C_{G}+C_{S}\right)}
$$

where $C^{\prime}$ is the real part capacitance (or simply, the capacitance) and $C^{\prime \prime}$ is the imaginary part capacitance (otherwise known as the dielectric loss).

From the complex capacitance formula, the expressions for real and imaginary capacitance can be calculated. This is achieved by multiplying the nominator and denominator by the complex conjugate of the denominator and then grouping to obtain the real $\left(C^{\prime}\right)$ parts (Eq. 29) and imaginary $\left(C^{\prime \prime}\right)$ parts (Eq. 30).

$$
\begin{gathered}
C^{*}=\frac{1}{i \omega Z^{*}}=\frac{\left(C_{G}+i \omega R_{S} C_{2} C_{G}\right)\left(1-i \omega R_{S}\left(C_{S}+C_{G}\right)\right)}{\left(1+i \omega R\left(C_{S}+C_{G}\right)\right)\left(1-i \omega R_{S}\left(C_{S}+C_{G}\right)\right)} \\
=\frac{C_{G}+\omega^{2} R_{S}^{2} C_{2 G} C_{S}\left(C_{S}+C_{G}\right)-i \omega R_{S} C_{G}^{2}}{1+\left(\omega R_{S}\left(\left(C_{S}+C_{G}\right)\right)^{2}\right.} \\
C^{\prime}=\frac{C_{G}+\omega^{2} R_{S}^{2} C_{G} C_{S}\left(C_{S}+C_{G}\right)}{1+\left(\omega R_{S}\left(\left(C_{S}+C_{G}\right)\right)^{2}\right.} \\
C^{\prime \prime}=-\frac{\omega R_{S} C_{G}^{2}}{1+\left(\omega R_{S}\left(\left(C_{S}+C_{G}\right)\right)^{2}\right.}
\end{gathered}
$$




\section{Glossary}

\section{List of Abbreviations}

ADC Analog digital converter

AWG American wire gauge

BDS Broadband dielectric spectroscopy

DAQ Data acquisition card

DSC Differential scanning calorimetry

DTA Differential thermal analysis

ER Electrical resistivity measurements

ETA Electrical thermal analysis

FDM Freeze drying microscope

IS Impedance spectroscopy

IVC Current to voltage converter

MW Maxwell-Wagner polarization process

OUT Object under test

TSC Thermally stimulated current spectroscopy

TVIS Through vial impedance spectroscopy

\section{List of Symbols}

- $\quad$ Series arrangement of two elements in an electrical circuit

$C^{\prime} \quad$ Real part capacitance or dielectric storage of complex capacitance

$C^{\prime \prime} \quad$ Imaginary part capacitance or dielectric loss of complex capacitance

$C^{\prime}(\infty) \quad$ Real part capacitance at high frequency

$C_{P E A K}^{\prime \prime} \quad$ Peak amplitude of the imaginary capacitance

$C_{f i t}^{\prime} \quad$ Real part capacitance from equivalent circuit modelling

$C_{f i t}^{\prime \prime} \quad$ Imaginary part capacitance from equivalent circuit modelling

$C_{a} \quad$ Capacitance of adhesive layer

$C_{a-g} \quad$ Capacitance of the composite glass wall and adhesive layer in series

$C_{a-G} \quad$ Total capacitance of the composite glass wall and adhesive layer in series

$C_{g} \quad$ Capacitance of glass-sample interface

$C_{G} \quad$ Total glass-sample interface capacitance

$C_{i} \quad$ Capacitance of the interfacial layer between glass and sample

$C_{o} \quad$ Capacitance of empty cell

$C_{s} \quad$ Capacitance of sample

$C_{S}(\infty)$ Capacitance of sample in the limit of high frequency

$C_{S}(0) \quad$ Capacitance of sample in the limit of low frequency

$C_{s}(f) \quad$ Capacitance of sample as a function of frequency

$C P E_{G} \quad$ Constant phase element of glass wall

$C_{f i t}^{\prime}(\infty)$ Real part capacitance from modelling at high frequency 
$C_{f i t}^{\prime}(f) \quad$ Real part capacitance from modelling as the function of frequency

$C_{f i t}^{\prime}(0) \quad$ Real part capacitance from modelling at low frequency

$D E_{S} \quad$ Distribution element of sample

$D E_{S}-R \quad$ Distribution element of sample describing resistance

$D E_{S}-T \quad$ Distribution element of sample describing time constant

$D E_{S}-U$ Distribution element of sample describing broadness

$F_{P E A K} \quad$ Peak frequency of the imaginary capacitance

$I_{O} \quad$ Current amplitude

$Q_{o} \quad$ Admittance of a constant phase element at an angular frequency of $\omega=1 \mathrm{rad} \mathrm{s}^{-1}$

$R_{S} \quad$ Resistance of sample

$T_{c} \quad$ Collapse temperature

$T_{e u} \quad$ Eutectic temperature

$T_{g} \quad$ Glass transition temperature

$T_{g}^{\prime} \quad$ Glass transition of the maximally freeze concentrated solution

$T_{\mathrm{m}} \quad$ Melting temperature

$T_{b} \quad$ Ice temperature at the base of a vial

$T_{i} \quad$ Ice temperature at the sublimation interface

$V_{o} \quad$ Voltage amplitude

$|Y| \quad$ Admittance magnitude

$Y_{C} \quad$ Admittance of a capacitor

$Y_{C P E} \quad$ Admittance of a constant phase element

$Y_{R} \quad$ Admittance of a resistor

$Z^{\prime} \quad$ Real part impedance

$Z^{\prime \prime} \quad$ Imaginary part impedance

$Z^{*} \quad$ Complex impedance

$Z_{C} \quad$ Impedance of capacitance

$Z_{C P E} \quad$ Impedance of constant phase element

$Z_{R} \quad$ Impedance of resistance

$d_{g} \quad$ Glass wall thickness

$k_{g} \quad$ Cell constant of glass

$k_{s} \quad$ Cell constant of sample

$\varepsilon_{\infty} \quad$ Permittivity in the limit of high frequency

$\varepsilon_{a} \quad$ Permittivity of adhesive

$\varepsilon_{g} \quad$ Permittivity of glass

$\varepsilon_{o} \quad$ Permittivity of free space

$\varepsilon_{r} \quad$ Relative permittivity

$\varepsilon_{S} \quad$ Static permittivity

$\rho_{S} \quad$ Sample resistivity

$\omega_{c} \quad$ Angular frequency at cross over between the dominance of two circuit elements

$|\mathrm{Z}| \sin \varphi \quad$ Imaginary part of the complex impedance or reactance 


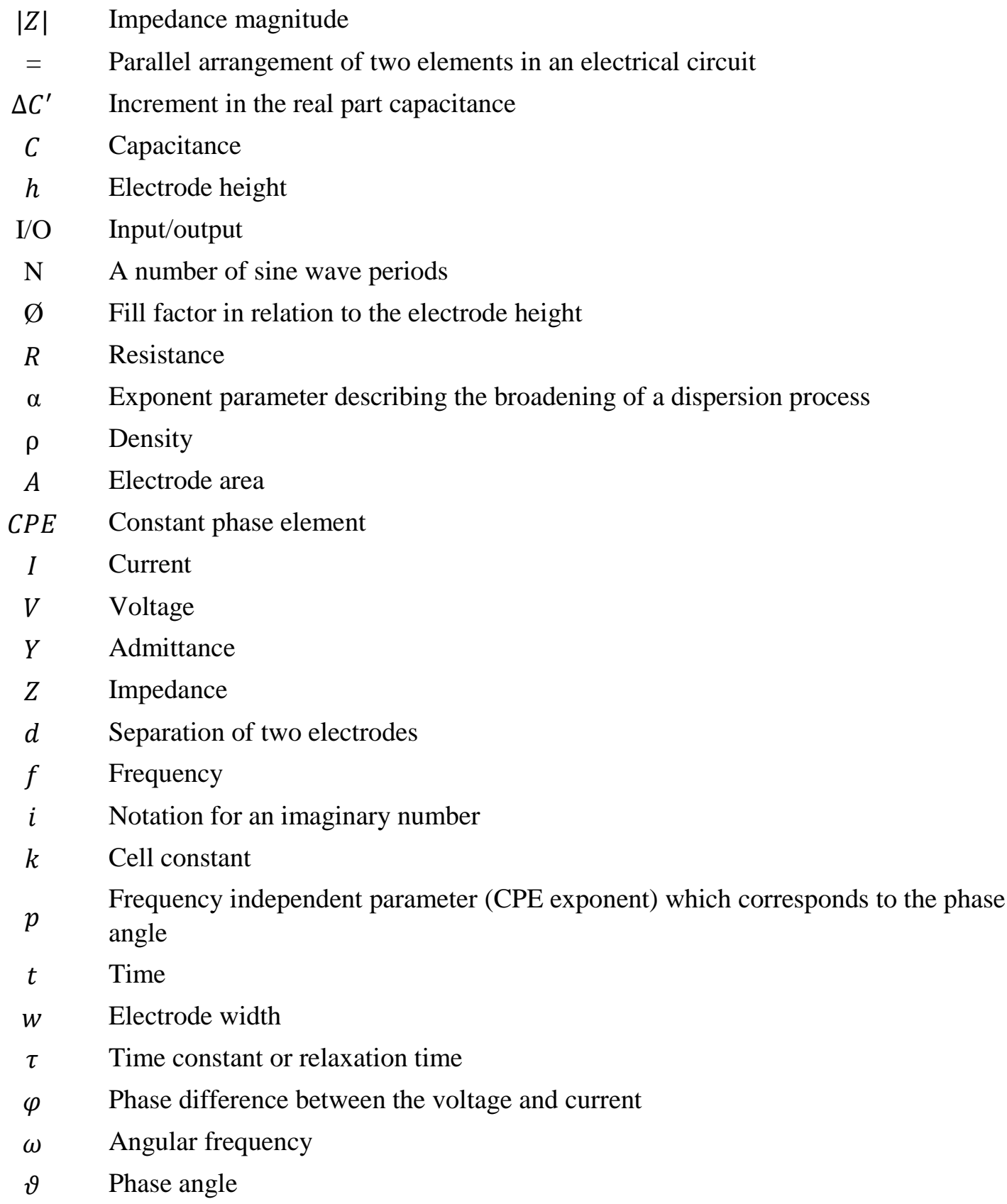

$\begin{array}{cl}{ }^{\circ} & \text { Degree } \\ { }^{\circ} \mathrm{C} & \text { Degree Celsius } \\ \mathrm{dB} & \text { Decibel } \\ \mathrm{F} \cdot \mathrm{m}^{-1} & \text { Farad per meter } \\ \mathrm{fF} & \text { Femtofarad } \\ \mathrm{g} & \text { Gram } \\ \mathrm{g} \cdot \mathrm{cm}^{-3} & \text { Gram per cubic centimeters } \\ \mathrm{G} \Omega & \text { Gigaohm or } 10^{9} \mathrm{ohm} \\ \mathrm{Hz} & \text { Hertz } \\ \mathrm{K} & \text { Kelvin }\end{array}$




$\begin{array}{cl}\mathrm{kg} \cdot \mathrm{m}^{-3} & \text { Kilogram per cubic meters } \\ \mathrm{kHz} & \text { Kilohertz or } 10^{3} \text { hertz } \\ \mathrm{MHz} & \text { Megahertz or } 10^{6} \mathrm{Hertz} \\ \mathrm{min} & \text { Minute } \\ \mathrm{mL} & \text { Millilitre } \\ \mathrm{mm} & \text { Millimetre } \\ \mathrm{mm}^{2} & \text { Square millimeter } \\ \mathrm{ms} & \text { Millisecond } \\ \mathrm{pF} & \text { picoFarad } \\ \mathrm{rad} \cdot \mathrm{s}^{-1} & \text { radians per second } \\ \mathrm{s} & \text { Second } \\ \mathrm{V} & \text { Volt } \\ \mu \mathrm{s} & \text { Microsecond } \\ \Omega & \text { Ohm }\end{array}$

